\title{
APPLICATIONS OF SIMPLICIAL $M$-SETS TO PROPER AND STRONG SHAPE THEORIES
}

\section{J. HERNÁNDEZ PARICIO}

\begin{abstract}
In this paper we have tried to reduce the classical classification problems for spaces and maps of the proper category and of the strong shape category to similar problems in the homotopy category of simplicial sets or in the homotopy category of simplicial $M$-sets, which $M$ is the monoid of proper selfmaps of the discrete space $\mathbb{N}$ of nonnegative integers.
\end{abstract}

Given a prospace (prosimplicial set) $Y$, we have constructed a simplicial set $\mathscr{\mathscr { P }}^{R} Y$ such that the Hurewicz homotopy groups of $\mathscr{\mathscr { P }}^{R} Y$ are the Grossman homotopy groups of $Y$. For the case of the end prospace $Y=\varepsilon X$ of a space $X$, we obtain Brown's proper homotopy groups; and for the Vietoris prospace $Y=V X$ (introduced by Porter) of a compact metrisable space $X$, we have Quigley's inward groups. The simplicial subset $\mathscr{\mathscr { P }}^{R} Y$ of a tower $Y$ contains, as a simplicial subset, the homotopy $\operatorname{limit} \lim ^{R} Y$. The inclusion $\lim ^{R} Y \rightarrow \overline{\mathscr{P}}^{R} Y$ induces many relations between the homotopy and (co)homology invariants of the prospace $Y$.

Using the functor $\overline{\mathscr{P}}^{R}$ we prove Whitehead theorems for proper homotopy, prohomotopy, and strong shape theories as a particular case of the standard Whitehead theorem. The algebraic condition is given in terms of Brown's proper groups, Grossman's homotopy groups and Quigley's inward groups, respectively. In all these cases an equivalent cohomological condition can be given by taking twisted coefficients.

The "singular" homology groups of $\overline{\mathscr{P}}^{R} Y$ provide homology theories for the Brown, Grossman and Quigley homotopy groups that satisfy Hurewicz theorems in the corresponding settings. However, there are other homology theories for the homotopy groups above satisfying other Hurewicz theorems.

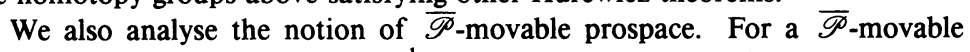
tower we prove easily (without $\lim ^{1}$ functors) that the strong homotopy groups agree with the Čech homotopy groups and the Grossman homotopy groups are determined by the Čech (or strong) groups by the formula $G_{\pi_{q}}=\overline{\mathscr{P}}_{\check{\pi}_{q}}$. This implies that the algebraic condition of the Whitehead theorem can be given in terms of strong (Čech) groups when the condition of $\mathscr{P}$-movability is included.

We also study homology theories for the strong (Steenrod) homotopy groups which satisfy Hurewicz theorems but in general do not agree with the corresponding Steenrod-Sitnikov homology theories.

Received by the editors April 14, 1993 and, in revised form, March 10, 1994.

1991 Mathematics Subject Classification. Primary 55N05, 55N07, 55N35, 55P55, 55Q07, $55 \mathrm{Q} 99$.

Key words and phrases. Model category, proper homotopy, prohomotopy, strong shape category, monoid, $M$-set, simplicial $M$-set, Edwards-Hastings embedding, Brown's $\mathscr{P}$ functor, proper realization, proper singular functor, Brown's proper homotopy groups, Grossman's homotopy groups, Quigley's inward groups, strong homotopy groups, Čech homotopy groups, Steenrod-Sitnikov homology. 


\section{INTRODUCTION}

The main purpose of this paper is to reduce as far as possible the study of proper homotopy theory and strong shape theory to notions and problems of standard homotopy theory. To reach this aim we will use the following tools:

(1) The notion of closed simplicial model category, introduced by Quillen [Q.1], will be used to work with categories obtained when one divides by homotopy relations or when one inverts weak equivalences.

(2) We consider the category $S S$ of simplicial sets and the category $S\left(S_{M}\right)$ of simplicial $M$-sets. An $M$-set is a set together with the action of a monoid $M$. Both categories $S S, S\left(S_{M}\right)$ are provided with structures of closed simplicial model categories.

(3) One of the most useful categories to study proper and strong shape theory is the closed simplicial model category of prospaces.

For the proper category at infinity, the Edwards-Hastings functor $\varepsilon$ gives a full embedding of the proper homotopy category at infinity of $T_{2}$ locally compact, $\sigma$-compact spaces into the localized category of prospaces obtained by inverting the weak equivalences considered by Edwards and Hastings in [E$\mathrm{H}]$. There is a similar version for global proper maps and homotopies by taking global (or augmented) prospaces. For strong shape theory, one can use the Vietoris functor $V$, introduced by Porter [P.1, P.5], that gives a full embedding of the strong shape category into the "homotopy category" of prospaces.

(4) We define "singular" functors from proper categories and procategories to simplicial $M$-sets, and realization functors from simplicial $M$-sets to the category of prospaces and from adequate full subcategories of simplicial $M$ sets to the category of spaces and proper maps.

One of the main results of this paper is the construction of a simplicial set $\overline{\mathscr{P}}^{R} X$, associated with a pro-simplicial set $X$, that retains many homotopy properties of $X$. The simplicial set $\overline{\mathscr{P}}^{R} X$ contains as a simplicial subset the homotopy $\operatorname{limit}, \lim ^{R} X$, of $X$.

The Hurewicz homotopy groups of $\lim ^{R} Y$ are the strong homotopy groups of the prosimplical set $Y$. For the case $Y=\varepsilon X$ one gets the strong groups in the proper setting, and for the case $Y=V X$ one has the approaching groups of Quigley [Quig, P.6].

In this paper, we prove that the Hurewicz homotopy groups of $\overline{\mathscr{P}}^{R} Y$ are the Grossman homotopy groups of the prospace $Y$. For $Y=\varepsilon X$ one has Brown's proper homotopy groups and for $Y=V X$ we have the "inward" groups of Quigley [Quig, P.6].

In the proper setting, the simplicial set $\overline{\mathscr{P}}_{\varepsilon X}^{R}$ can be interpreted as the simplicial set of sequences of singular $q$-simplexes converging to infinity. We can also look at $\overline{\mathscr{P}}^{R}{ }_{\varepsilon X}$ as the mapping space of sequences of points of $X$ converging to infinity provided with an adequate topology. If $X$ is a compact metrisable space, it can be considered up to homeomorphism as a subspace of the pseudointerior of the Hilbert cube $Q$. We can then interpret $\overline{\mathscr{P}}^{R} V X$ as the simplicial set of sequences of singular $q$-simplexes of $Q$ converging to $X$. Nevertheless, it is also possible to interpret $\overline{\mathscr{P}}^{R} V X$ as the simplicial set of singular $q$-simplexes of $Q-X$ converging to $X$, which is the same as the 
simplicial set of singular $q$-simplexes of $Q-X$ converging to infinity. That is, $\overline{\mathscr{P}}^{R} V X$ is isomorphic to $\mathscr{\mathscr { P }}^{R} \varepsilon(Q-X)$ in the homotopy category.

This paper has been divided into 10 sections. The first part, $\S \S 1$ through 6 , is devoted to developing the technical tools. Section 7 establishes the relationships between simplicial $M$-sets and simplicial complexes, where $M$ is the monoid of proper selfmaps of the discrete space of natural numbers. The last part, $\S \S 8,9$ and 10 , contains applications to proper homotopy theory, prohomotopy theory and strong shape theory.

Section 2 is devoted to analysing the closed simplicial model structure of simplicial $M$-sets that will be used in this paper. In $\S 3$, we analyse realization and singular functors for simplicial $M$-sets. Let $\Delta$ denote the standard category whose objects are finite ordered sets of the form $[q]=\{0<1<\cdots<q\}$ and the morphisms are monotone maps. Let ${ }_{M} \mathscr{C}$ be the category of left $M$-objects in $\mathscr{C}$. Associated with a functor $\chi: \Delta \rightarrow{ }_{M} \mathscr{C}$, we consider a realization functor $R_{\chi}: S\left(S_{M}\right) \rightarrow \mathscr{C}$ and a singular functor $S_{\chi}: \mathscr{C} \rightarrow S\left(S_{M}\right)$. The construction of the realization functor depends of the existence of colimits in $\mathscr{C}$. One of the categories $\mathscr{C}$ that we consider is the category Pro of spaces and proper maps. The category Pro has only some colimits, and for this reason, in $\S 3$ we include some lemmas about the existence of colimits.

If we consider the monoid $M=\operatorname{Pro}(\mathbb{N}, \mathbb{N})$, we take as a "proper" $q$-simplex a space of the form $\mathbb{N} \times|\Delta[q]|$. Given a proper map $\varphi: \mathbb{N} \rightarrow \mathbb{N}$, we can attach $\mathbb{N} \times\left|\partial_{i} \Delta[q]\right|$ to $\mathbb{N} \times|\Delta[q-1]|$ in such a way that $\{n\} \times\left|\partial_{i} \Delta[q]\right|$ is identified with $\{\varphi(n)\} \times|\Delta[q-1]|$. Therefore if we have a simplicial $M$-set $N$, whose monoid structure is freely generated by a finite number of simplexes, we can construct a space $R_{p} N$ taking a "proper" simplex associated with each generator of $N$ and gluing the different "proper" simplexes in the way indicated above. The full subcategory of this kind of simplicial $M$-sets is denoted by $S\left(S_{M} / f f\right) / f d$ ( $f f=$ freely generated by a finite set, $f d=$ finite dimension). In general the realization of a simplicial map of $S\left(S_{M}\right)$ is not proper, but the realization of a simplicial map of $S\left(S_{M} / f f\right) / f d$ is proper.

The main result of $\S 4$ establishes that if $N$ is an object of $S\left(S_{M} / f f\right) / f d$ which is cofibrant and $Y$ is a topological space, then the set of proper homotopy classes $\pi_{0}$ (Pro) $\left(R_{p} N, Y\right)$ is isomorphic to the hom-set $\operatorname{Ho}\left(S\left(S_{M}\right)\right)\left(N, S_{p} Y\right)$. It is interesting to remark that in $\S 7$ we have proved that a locally finite simplicial complex $X$ which has finite dimension and a countably infinite number of simplexes is always of the form $X \cong R_{p} N$.

For the category pro $S S$, we think of as a $q$-simplex, the pro-simplicial set $c \Delta[q]$ which is defined by $c \Delta[q](i)=\sum_{j \geq i} \Delta[q], i \in \mathbb{N}$. Similar notions of $q$ simplex are considered for global prosimplicial sets and for the corresponding pointed cases. Associated with these $q$-simplexes, there are a realization functor $R_{\chi_{\infty}}: S\left(S_{M_{\infty}}\right) \rightarrow \operatorname{pro} S S$ and a singular functor $S_{\chi_{\infty}}: \operatorname{pro} S S \rightarrow S\left(S_{M_{\infty}}\right)$.

In 1975, E. M. Brown [Br.1] defined the proper homotopy groups ${ }^{B} \pi_{q}^{\infty}(X)$ of a $\sigma$-compact space $X$ with a base ray. He also considered a functor $\mathscr{P}$ : tow $\mathrm{Gps} \rightarrow$ Gps which carries the tower of homotopy groups, $\pi_{q} \varepsilon X$, of a tower of neighbourhoods of $X$ at infinity to the homotopy group ${ }^{B} \pi_{q}^{\infty}(X)$. For the case $q=0, \overline{\mathscr{P}}$ is a functor from tow Set $_{*}$ to Set $_{*}$. Here we consider new versions of the $\overline{\mathscr{P}}$ functor which are of the form proSet $\rightarrow \operatorname{Set}_{\mathscr{P}_{C *}}, \operatorname{proSet}_{*} \rightarrow$ $\operatorname{Set}_{\mathscr{P} c S^{0}}$, proGps $\rightarrow \mathrm{Gps}_{\mathscr{P} c \mathbb{Z}}$, etc. The new versions are provided with the 
additional structure of the action of a monoid $\left(\mathscr{P}_{c *}, \mathscr{P}_{c} S^{0}\right)$ or a near-ring $\mathscr{P} c \mathbb{Z}$.

Using the shorter notation $S=$ Set,$S_{*}=\operatorname{Set}_{*}$, the functor $\mathscr{P}:$ pro $S \rightarrow$ $S_{\mathscr{P}_{C *}}$ induces a functor $S \mathscr{P}: S$ pro $S \rightarrow S\left(S_{\mathscr{P}_{C *}}\right)$ and we have the composite pro $S S \stackrel{F}{\rightarrow} S$ pro $S \stackrel{S \mathscr{P}}{\rightarrow} S\left(S_{\mathscr{P}_{C *}}\right)$, where $F$ is naturally defined. The main result of $\S 5$ establishes that $S \mathscr{P} F$ is isomorphic to the "singular" functor $S_{\chi_{\infty}}$. That is, $S_{\chi_{\infty}}$ is an extension of the $\mathscr{P}$ functor and, for this reason, the functor $S_{\chi_{\infty}}$ is also denoted by $\mathscr{P}$.

The inverse limit functor lim: tow $S S \rightarrow S S$ is related with the functor $\mathscr{P}:$ tow $S S \rightarrow S\left(S_{\mathscr{P}_{\mathcal{C}}}\right)$ in the following way: There is an element sh in the monoid $\mathscr{P}_{C} *$ such that the simplicial subset $F_{\mathrm{sh}} X=\left\{x \in \mathscr{P}_{X} \mid x \mathrm{sh}=x\right\}$ of elements fixed by sh is isomorphic to $\lim X$, where $X$ is an object in tow $S S$.

Section 6 contains the main technical results of this paper. It is well known that the homotopy inverse limit, holim, can be defined as a right-derived functor of the lim functor. We prove that the functor $\mathscr{P}: \operatorname{pro} S S \rightarrow$ pro $S\left(S_{\mathscr{P}_{C *}}\right)$ has a right-derived functor $\mathscr{P}^{R}: \mathrm{Ho}(\operatorname{pro} S S) \rightarrow \mathrm{Ho}\left(S\left(S_{\mathscr{P}_{c^{*}}}\right)\right)$. The relation above between lim and $\mathscr{P}$ induces the formula $\lim ^{R} X=F_{\mathrm{sh}} \mathscr{P}^{R} X=\left\{x \in \mathscr{P}^{R} X \mid x \mathrm{sh}=\right.$ $x$ \} where $X$ is an object in tow $S S$.

We summarize the results of $\S 6$ by saying that there is a pair of adjoint functors

$$
\operatorname{Ho}(\operatorname{pro} S S) \underset{\mathscr{P}^{R}}{\stackrel{\mathscr{L}^{L}}{\leftrightarrows}} \mathrm{Ho}\left(S\left(S_{\mathscr{P}_{C *}}\right)\right) \text {. }
$$

that can be composed with the pair of adjoint functors (given in $\S 2$ )

$$
\operatorname{Ho}\left(S\left(S_{\mathscr{P}_{C *}}\right)\right) \underset{U}{\stackrel{-\otimes \mathscr{P}_{c *}}{\leftrightarrows}} \mathrm{Ho}(S S)
$$

to obtain the new pair

$$
\mathrm{Ho}(\operatorname{pro} S S) \underset{\overline{\mathscr{P}}^{R}}{\stackrel{\overline{\mathscr{L}}^{L}}{\rightleftarrows}} \mathrm{Ho}(S S) \text {. }
$$

A first consequence of the existence of these pairs of adjoint functors is that the Hurewicz homotopy groups of $\mathscr{P}^{R} X(X$ is an object in pro $S S)$ are isomorphic to the Grossman homotopy groups of $X$. We also prove that $\pi_{q}$ and $\overline{\mathscr{P}}$ "commute"; that is, $\pi_{q} \overline{\mathscr{P}}^{R} X \cong \overline{\mathscr{P}}_{q} X$. This proves Brown's result that $\overline{\mathscr{P}}$ carries the towers of homotopy groups, $\pi_{q} \varepsilon X$, to the proper homotopy groups, ${ }^{B} \pi_{q}^{\infty} X$. As a second consequence, we will translate some classical theorems of standard homotopy theory to prohomotopy theory.

Section 8 is devoted to obtaining some applications to proper homotopy theory. We use the functors $R_{p}$ and $S_{p}$ to transform classical theorems of standard homotopy theory into similar theorems in the proper setting. We analyse two Examples. In the first the proper Whitehead theorem is proved as a particular case of the standard Whitehead theorem. We also remark that the cohomology version of the Whitehead theorem with twisted coefficients implies a cohomology version in the proper setting. In the second example, we see how the standard Hurewicz theorem implies a Hurewicz theorem in the proper category. This method also provides a proper homology theory for the Brown proper ho- 
motopy groups. However, there are other proper homology theories that also satisfy Hurewicz theorems for the Brown proper groups see-Remark 3 after Theorem 7.3. In this section we also analyse the relation between the proper singular functor, the right-derived functor of the $\mathscr{P}$ functor and the EdwardsHastings functor $\varepsilon$. As a consequence of this relation, we obtain a partial version of the Edwards-Hastings embedding theorem for the proper category.

In $\S 9$, we have developed some applications to prohomotopy theory. We introduce the notion of $\mathscr{L}$-cofibrant prospace (or prosimplicial set). The class of $\mathscr{L}$-cofibrant prospaces contains the end prospace $\varepsilon X$ of a finite-dimensional simplicial complex and Porter's Vietoris prospace $V X$ of a compact metrisable space $X$ which has finite covering dimension. Therefore any result about $\mathscr{L}$ cofibrant prospaces has interpretations in the proper and in the strong shape settings. In this section, we establish a Whitehead theorem for the class of $\mathscr{L}$ cofibrant prospaces. Using the functors $\mathscr{L}^{L}$ and $\mathscr{P} R$, we obtain this result as a particular case of the standard Whitehead theorem. The algebraic condition of the theorem is given in terms of the Grossman homotopy groups. There is also an equivalent cohomology condition.

We also introduce a notion of $\overline{\mathscr{P}}$-movability that in general is weaker than the notion of movable given in [E-H]. We give an easy proof (without using $\lim ^{1}$ functors) that for $\overline{\mathscr{P}}$-movable towers, the strong (Steenrod) homotopy groups are isomorphic to the Čech homotopy groups and the Grossman groups are also determined by the formula ${ }^{G} \pi_{q}=\mathscr{\mathscr { P }}\left({ }^{S} \pi_{q}\right)$. Therefore for $\mathscr{L}$-cofibrant $\overline{\mathscr{P}}$-movable towers the algebraic condition of the Whitehead theorem can be expressed in terms of strong (Steenrod) homotopy groups or Čech homotopy groups.

Section 10 is devoted to obtaining some applications to strong shape theory. Recall that the Grossman homotopy groups of the prospace $V X$ are the Quigley [Qui] inward groups (see also [P.6]). Using the functor $\overline{\mathscr{P}}^{R}$, the Quigley inward groups are interpreted as the Hurewicz homotopy groups of the simplicial set $\mathscr{\mathscr { P }}^{R} V X$. Therefore defining the homology groups of $X$ as the "singular" homology of $\overline{\mathscr{P}}^{R} V X$, we obtain a homology theory that satisfies the Hurewicz theorem for the Quigley inward groups. Nevertheless, in Remark (4) after Definition 10.1, we suggest other homology theories for the Quigley inward groups. In general $H_{q} \overline{\mathscr{P}}^{R} V X$ is not isomorphic to $\overline{\mathscr{P}} H_{q} V X$, but there are other homology theories such that $H$ "commutes" with the $\overline{\mathscr{P}}$ functor.

It is also known that the strong (Steenrod) homotopy groups of the prospace $V X$ are the Quigley approaching groups. Using the functor $\mathscr{P} R$, we have that the Quigley approaching groups of a compact metrisable space $X$ are the Hurewicz homotopy groups of $F_{\mathrm{sh}} \mathscr{P}^{R} V X$. Therefore we obtain a Hurewicz theorem if we define the homology of $X$ as the "singular" homology of $F_{\mathrm{sh}} \mathscr{P}^{R} V X$. This gives a nice homology for Quigley's approaching groups that is not isomorphic to the strong (Steenrod) homology groups used by Kodama and Koyama [K-K] to obtain a Hurewicz theorem for these groups.

We finish the paper by giving a Whitehead theorem for the strong shape category in terms of Quigley inward groups. For the case of $\overline{\mathscr{P}}$-movable spaces the algebraic condition can also be given in terms of Quigley approaching groups or Čech groups. 


\section{Closed Simplicial model CATEgories}

The tool used in this paper is the notion of closed simplicial model category. We refer the reader to [Q.1] and [Q.2], which contain the necessary definitions, examples, and the main properties of this structure.

Given a solid arrow diagram in a category $C$

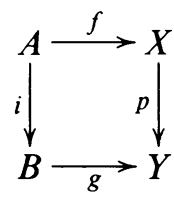

it is said that $i$ has the left lifting property (LLP) with respect to $p$ and $p$ is said to have the right lifting property (RLP) with respect to $i$ if there exists a map $h: B \rightarrow X$ such that $h i=f$ and $p h=g$.

A closed model category is a category $C$ endowed with three distinguished families of maps, called cofibrations, fibrations, and weak equivalences, satisfying certain axioms.

These axioms were considered in [Q.1], and an equivalent but different formulation was given in [Q.2].

Given a closed model category $C$, the homotopy category $\mathrm{Ho}(C)$ is obtained from $C$ by formally inverting all the weak equivalences (see [Q.1] and [G-Z]).

A simplicial category is a category $C$ endowed with a functor $\operatorname{Hom}_{C}: C^{\mathrm{op}} \times$ $C \rightarrow S S$ satisfying the axioms given in [Q.1]; in particular we have that $\operatorname{Hom}_{C}(X, Y)_{0} \cong C(X, Y)$. Associated with a simplicial category $C$, we have the category $\pi_{0} C$ which has the same objects as $C$ and the hom-set defined by $\pi_{0} C(X, Y)=\pi_{0} \operatorname{Hom}_{C}(X, Y)$, where $\pi_{0} \operatorname{Hom}(X, Y)$ is the set of connected components of the simplicial set $\operatorname{Hom}_{C}(X, Y)$.

A closed simplicial model category is a simplicial category which is also a closed model category and satisfies certain axioms, see [Q.1]. For a finite simplicial set $K$ a closed simplicial category $C$ is provided with objects $X \otimes K$, $X^{K}$ for any object $X$ in $C$. Associated with these objects, there are the following isomorphisms:

$$
\begin{gathered}
\operatorname{Hom}_{C}(X \otimes K, Y) \cong \operatorname{Hom}_{S S}\left(K, \operatorname{Hom}_{C}(X, Y)\right), \\
\operatorname{Hom}_{C}\left(X, Y^{K}\right) \cong \operatorname{Hom}_{S S}\left(K, \operatorname{Hom}_{C}(X, Y)\right) .
\end{gathered}
$$

Suppose that $C$ is a closed simplicial model category and $\varnothing$ denotes the initial object and $*$ denotes the final object. An object $X$ is said to be cofibrant if the unique map $\varnothing \rightarrow X$ is a cofibration, and an object $Y$ is said to be fibrant if the unique map $Y \rightarrow *$ is a fibration.

The main relation between the categories $\pi_{0} C$ and Ho $C$ is given through cofibrant and fibrant objects: If $X$ is cofibrant and $Y$ is fibrant, then

$$
\pi_{0} C(X, Y) \cong \mathrm{Ho} C(X, Y) .
$$

It is said that $C$ is a pointed category if both the initial and final objects exist and are isomorphic. In this case, for two objects $X, Y$ in $C$, we always have the zero map $*: X \rightarrow Y$ that defines a 0 -simplex of $\operatorname{Hom}_{C}(X, Y)$. Therefore we also have a natural functor $\mathrm{Hom}_{C}: C^{\mathrm{op}} \times C \rightarrow S S_{*}$. 
Examples. (1) The category $S S$ of simplicial sets. Let $\Delta[n]$ denote the standard $n$-simplex, $\dot{\Delta}[n]$ the simplicial set generated by the faces of $\Delta[n]$, and $V(n, k)$ for $0 \leq k \leq n>0$ the simplicial subset of $\Delta[n]$ generated by the $(n-1)$-faces $\partial_{i}: \Delta[n-1] \rightarrow \Delta[n]$ with $0 \leq i \leq n$ and $i \neq k$. A map $f: X \rightarrow Y$ is said to be a fibration if for all $n>0$ it has the RLP with respect to $V(n, k) \rightarrow \Delta[n], 0 \leq k \leq n$. A map $f: X \rightarrow Y$ is said to be a trivial fibration if $f$ has the RLP with respect to $\dot{\Delta}[n] \rightarrow \Delta[n], n \geq 0$. A map $i: A \rightarrow B$ is said to be a cofibration (resp. trivial cofibration) if $i$ has the RLP with respect to any trivial fibration (resp. fibration). A map $f$ is said to be a weak equivalence if $f$ can be factored as $f=p i$ where $i$ is a trivial cofibration and $p$ is a trivial fibration.

Given a simplicial set $K$, the object $X \otimes K$ is defined to be $X \otimes K=X \times K$.

The functor $\operatorname{Hom}_{S S}: S S^{\text {op }} \times S S \rightarrow S S$ is defined by $\operatorname{Hom}_{S S}(X, Y)_{n} \cong$ $S S(X \otimes \Delta[n], Y)$. The object $X^{K}$ is defined by $X^{K}=\operatorname{Hom}_{S S}(K, X)$.

The category of pointed simplicial sets $S S_{*}$ is also a closed simplicial model category. If we consider the functor ()$^{+}: S S \rightarrow S S_{*}$ which carries $X$ to $X \sqcup *$, we have that ()$^{+}$is the left adjoint of the forgetful functor $U: S S_{*} \rightarrow$ $S S$. A map $f$ is said to be a fibration (resp. weak equivalence) if $U f$ is a fibration in $S S$ (resp. weak equivalence). A map is a cofibration if it has the LLP with respect to trivial fibrations.

For objects $X$ in $S S_{*}$ and $K$ in $S S$, define

$$
X \otimes K \cong X \times K^{+} /\left((X \times *) \cup\left(* \times K^{+}\right)\right) .
$$

$\operatorname{Hom}_{S S_{*}}: S S_{*}^{\mathrm{op}} \times S S_{*} \rightarrow S S_{*}$ is defined by

$$
\operatorname{Hom}_{S S_{*}}(X, Y)_{n}=S S_{*}(X \otimes \Delta[n], Y),
$$

and $X^{K}=\operatorname{Hom}_{S S_{*}}\left(K^{+}, X\right)$.

(2) The category Top of topological spaces. Let $R: S S \rightarrow$ Top and $S:$ Top $\rightarrow S S$ be the realization and singular functors, respectively. A map $f: X \rightarrow Y$ in Top is said to be a fibration (weak equivalence) if $S f$ is a fibration (weak equivalence) in $S S$. A map $i: A \rightarrow B$ is a cofibration if $i$ has the LLP with respect to trivial fibrations. Given a finite simplicial set $K$ and a topological space $X$, the objects $X \otimes K$ and $X^{K}$ are defined by

$$
X \otimes K=X \times R K, \quad X^{K}=\operatorname{Top}(R K, X),
$$

where $R K$ is the realization of $K$ and $\operatorname{Top}(R K, X)$ is the mapping space of continuous maps from $R K$ to $X$ endowed with the compact-open topology. The functor Hom $_{\text {Top }}$ : Top ${ }^{\text {op }} \times$ Top $\rightarrow S S$ is defined by

$$
\operatorname{Hom}_{\text {Top }}(X, Y)_{n}=\operatorname{Top}(X \times R \Delta[n], Y) \text {. }
$$

The category $\mathrm{Top}_{*}$ of pointed spaces also admits a closed simplicial model structure. In this case for a given simplicial set $K$ and a pointed space $X$, the objects $X \otimes K$ and $X^{K}$ are defined by

$$
\begin{gathered}
X \otimes K=X \times(R K)^{+} /\left((X \times *) \cup\left(* \times(R K)^{+}\right)\right), \\
X^{K}=\operatorname{Top}_{*}\left((R K)^{+}, X\right),
\end{gathered}
$$

where $(R K)^{+}$is the disjoint union of $R K$ and the one-point space $(*)$.

(3) The category pro $C$ of pro-objects in $C$. Associated with a category $C$, we can consider the category pro $C$ introduced by A. Grothendieck [Gro]. 
A study of some properties of this category can be found in the appendix of [A-M], the monograph [E-H], or the books [M-S] and [C-P].

The objects of pro $C$ are functors $X: I \rightarrow C$, where $I$ is a small left filtering category and the set of morphisms from $X: I \rightarrow C$ to $Y: J \rightarrow C$ is given by the formula

$$
\text { pro } C(X, Y)=\lim _{j} \operatorname{colim} C\left(X_{i}, Y_{j}\right) .
$$

Edwards and Hastings [E-H] have proved that if $C$ has the structure of a closed simplicial model category and $C$ satisfies the condition $N$ (see [E-H, page 45]), then pro $C$ inherits a natural structure of a closed simplicial model category. For a given finite simplicial set $K$ and an object $X=\left\{X_{i}\right\}$ of pro $C$, the objects $X \otimes K$ and $X^{K}$ are defined by

$$
\left\{X_{i}\right\} \otimes K=\left\{X_{i} \otimes K\right\}, \quad\left\{X_{i}\right\}^{K}=\left\{X_{i}^{K}\right\} .
$$

The functor $\operatorname{Hom}_{\text {pro } C}$ : (pro $\left.C\right)^{\text {op }} \times$ pro $C \rightarrow S S$ is defined by

$$
\operatorname{Hom}_{\text {pro } C}(X, Y)_{n}=\operatorname{pro} C(X \otimes \Delta[n], Y) .
$$

\section{THE CATEGORY OF SIMPLICIAL $M$-SETS}

A monoid consists of a set $M$ and an associative multiplication: $M \times M \rightarrow$ $M,\left(m, m^{\prime}\right) \rightarrow m m^{\prime}$, with unit element $1(1 m=m=m 1$, for every $m \in M)$. A 0 -monoid $M$ is a monoid with a zero element $0 \in M \quad(m 0=0=0 m$, for every $m \in M)$. If $C$ is a category and $X$ is an object of $C$, then the hom-set $C(X, X)$ with the composition of morphisms, $(g, f) \rightarrow g f$, has a natural monoid structure. If $C$ is a category with zero object, then $C(X, X)$ is a 0-monoid.

Examples. (1) Let Pro be the category of spaces and proper maps (a continuous map is proper if the inverse image of a closed compact subset is compact) and consider the set of natural numbers $\mathbb{N}$ provided with the discrete topology. The set of proper maps $M=\operatorname{Pro}(\mathbb{N}, \mathbb{N})$ has a natural monoid structure. Let $A, B$ be closed subsets of a space $X$ and assume that $\operatorname{cl}(X-A), \operatorname{cl}(X-B)$ are compact. Given two proper maps $f: A \rightarrow Y, g: B \rightarrow Y$, it is said that $f$ and $g$ have the same germ if there exists a closed subset $C$ of $X$ such that $\operatorname{cl}(X-C)$ is compact, $C \subset A, C \subset B$ and $f / C=g / C$. Let Pro $_{\infty}$ denote the category of spaces and germs of proper maps. The monoid of germs of proper maps $M_{\infty}=\operatorname{Pro}_{\infty}(\mathbb{N}, \mathbb{N})$ will also be consider in this paper.

(2) Let $\widehat{\mathbb{N}}=\mathbb{N} \cup\{\infty\}$ be the Alexandroff compactification of $\mathbb{N}$. Taking $\infty$ as a base point, $\widehat{\mathbb{N}}$ becomes a pointed set. The endomorphism pointed set $\operatorname{Top}_{*}(\widehat{\mathbb{N}}, \widehat{\mathbb{N}})$ has a natural 0 -monoid structure. Two pointed continuous maps $f, g: \widehat{\mathbb{N}} \rightarrow \widehat{\mathbb{N}}$ have the same germ at $\infty$ if there is $n_{0} \in \mathbb{N}$ such that $f(n)=g(n)$ for every $n \geq n_{0}$. The set $\operatorname{Top}_{*}^{\infty}(\widehat{\mathbb{N}}, \widehat{\mathbb{N}})$ of germs at $\infty$ of continuous maps from $\widehat{\mathbb{N}}$ to $\widehat{\mathbb{N}}$ also becomes a 0 -monoid.

Let $M$ be a monoid and $C$ a category. A left $M$-object $X$ in $C$ consists of an object $X$ of $C$ and a monoid homomorphism $M \rightarrow C(X, X): m \rightarrow$ $\widetilde{m}: X \rightarrow X$. If $M$ is a 0 -monoid and $C$ is a category with zero object, we suppose that an $M$-object $X$ in $C$ satisfies the additional condition $\tilde{0}=0$. The category whose objects are the (left) $M$-objects in $C$ will be denoted by 
${ }_{M} C$. By considering monoid "antimorphisms" $M \rightarrow C(X, X)$ we have the notion of right $M$-object in $C$ and the category $C_{M}$.

Let $C$ be a category; for each object $X$ of $C$ we have the monoid (or 0monoid) $M=C(X, X)$. If $Y$ is an object of $C$, the monoid "antimorphism"

$$
C(X, X) \rightarrow \operatorname{Set}(C(X, Y), C(X, Y)): \varphi \rightarrow \tilde{\varphi}, \tilde{\varphi}(f)=f \varphi, \quad f \in C(X, Y),
$$

induces the structure of a right $M$-set on $C(X, Y)$. Therefore we have a functor $C(X,-): C \rightarrow \operatorname{Set}_{M}$. If $C$ is pointed ( $C$ has a zero object) we get a functor $C(X,-): C \rightarrow \operatorname{Set}_{* M}$. Similarly, there are functions $C(-, Y): C \rightarrow$ ${ }_{M}$ Set or $C(-, Y): C \rightarrow{ }_{M}$ Set $_{*}$.

Examples. (1) For the category Pro and $M=\operatorname{Pro}(\mathbb{N}, \mathbb{N})$, we have the right $M$-set $\operatorname{Pro}(\mathbb{N}, X)$ of sequences in $X$ converging to infinity. Similarly for $M_{\infty}=\operatorname{Pro}_{\infty}(\mathbb{N}, \mathbb{N})$ we have the right $M_{\infty}$-set $\operatorname{Pro}_{\infty}(\mathbb{N}, X)$. If $|\Delta[q]|$ denotes the realization of the standard $q$-simplex, then we also get the right $M$-set $\operatorname{Pro}(\mathbb{N} \times|\Delta[q]|, X)$.

(2) Let $s=\prod_{n=1}^{+\infty}\left(\frac{-1}{n}, \frac{1}{n}\right)$ be the pseudo-interior of the Hilbert cube $Q=$ $\prod_{n=1}^{+\infty}\left[\frac{-1}{n}, \frac{1}{n}\right]$. Let $X$ be a compact subset of $s$. A sequence $x: \mathbb{N} \rightarrow Q$ converges to $X$ if for every neighbourhood $U$ of $X$ in $Q$, there is $n_{0}$ such that $x_{n} \in U$ for every $n \geq n_{0}$. The sets $\{x: \mathbb{N} \rightarrow X \mid x$ converges to $X\}$ and $\{x: \mathbb{N} \rightarrow Q-X \mid x$ converges to $X\}$ become $M$-sets for $M=\operatorname{Pro}(\mathbb{N}, \mathbb{N})$. Consider also sequences of simplexes $x: \mathbb{N} \times|\Delta[q]| \rightarrow Q$ converging to $X$; that is, for every neighborhood $U$ of $X$ in $Q$, there is $n_{0}$ such that $x(\{n\} \times|\Delta[q]|) \subset U$ for every $n \geq n_{0}$. The sets $s s_{q}(X)=\{x: \mathbb{N} \times|\Delta[q]| \rightarrow Q \mid x$ converges to $X\}$ and $s s_{q}^{c}(X)=\{x: \mathbb{N} \times|\Delta[q]| \rightarrow Q-X \mid x$ converges to $X\}$ become $M$-sets for $M=\operatorname{Pro}(\mathbb{N}, \mathbb{N})$ and $s s(X), s s^{c}(X)$ are simplicial $M$-sets associated with $X$.

Given a 0 -monoid $M$, the category of right $M$-pointed sets, Set $_{* M}$, is an algebraic category, see [Pa], by considering one 0 -ary operation to fix a base point $*$ and a 1-ary operation $m$ for each $m \in M$. The relations are given by $x 1=x, x 0=*,(x m) n=x(m n)$. In the case of a monoid, we do not need the 0 -ary operation and the relation $x 0=*$. By general properties of algebraic categories we have that $\operatorname{Set}_{* M}$ (resp., Set ${ }_{M}$ ) is a complete and cocomplete category, see [Pa; page 140]. That is, the category $\operatorname{Set}_{* M}\left(\operatorname{Set}_{M}\right)$ has limits and colimits. The categories of the form $\operatorname{Set}_{* M}, \operatorname{Set}_{M}$ enjoy very nice properties such as the existence of exponentials and a subobject classifier. That is, these categories are elementary topoi, see [M-M].

For these categories there is a natural forgetful functor $U:$ Set $_{* M} \rightarrow$ Set $_{*}$ (resp., $U:{ }_{M}$ Set $_{*} \rightarrow$ Set $_{*}$ ) and a left adjoint functor $-\odot M:$ Set $_{*} \rightarrow$ Set $_{* M}$ defined by $X \odot M=X \times M /(X \times 0 \cup * \times M)$. An element $(x, m) \in X \times M$ determines a unique class in $X \odot M$ that will be denoted by $x \odot m$. The forgetful functor Set $_{* M} \rightarrow$ Set $_{*}$ is faithful and preserves limits, and the left adjoint functor $-\odot M$ preserves colimits. For the nonpointed case, the forgetful functor Set $_{M} \rightarrow$ Set has also a left adjoint functor $-\odot M$ : Set $\rightarrow \operatorname{Set}_{M}$ which is defined by $X \odot M=X \times M$. In this case we also denote an element $(x, m)$ by $x \odot m$. We note that $X \odot M$, the free $M$-set generated by $X$, is isomorphic to $\sqcup_{x \in X} M$ provided with the canonical right action of $M$.

Let $\mathscr{C}$ be a category closed under finite limits. A map $f: X \rightarrow Y$ is said to 
be an effective epimorphism if for any object $T$ of $\mathscr{C}$, the diagram of sets

$$
\mathscr{C}(Y, T) \stackrel{f^{*}}{\longrightarrow} \mathscr{C}(X, T) \underset{p r_{2}^{*}}{\stackrel{p r_{1}^{*}}{\longrightarrow}} \mathscr{C}(X \underset{Y}{\times} X, T)
$$

is a difference kernel. An object $P$ of $\mathscr{C}$ is said to be projective if $\mathscr{C}(P, X) \stackrel{f^{*}}{\rightarrow}$ $\mathscr{C}(P, Y)$ is surjective whenever $f: X \rightarrow Y$ is an effective epimorphism. A category $\mathscr{C}$ has sufficiently many projectives, if for any object $X$, there is an effective epimorphism $P \rightarrow X$ where $P$ is a projective object. Assume that $\mathscr{C}$ is closed under colimits; an object $X$ is said to be small if $\mathscr{C}(X,-)$ commutes with filtered colimits. A class $\mathscr{U}$ of objects of $\mathscr{C}$ is a class of generators if, for every object $X$, there is an epimorphism $Q \rightarrow X$ where $Q$ is a sum of copies of members of $\mathscr{U}$.

For the case $\mathscr{C}=\operatorname{Set}_{* M}$ (resp., Set ${ }_{M}$ ) the class of effective epimorphisms is the class of set-theoretically surjective maps. Note that the category $\operatorname{Set}_{* M}$ (resp., Set ${ }_{M}$ ) has a class of generators, $\mathscr{U}$, with a single object, $S^{0} \odot M \cong M$ $(* \odot M \cong M)$. For later applications we also note that $M$ is projective and small.

If $\mathscr{C}$ is a category, let $S \mathscr{C}$ denote the category of simplicial objects in $\mathscr{C}$. We also have a natural functor in: $\mathscr{C} \rightarrow S \mathscr{C}$ which carries an object $A$ to the simplicial object in $A$ defined by (in $A)_{q}=A$ and where degeneracy and face operators are equal to the identity of $A$.

Quillen [Q.1] proved that if $\mathscr{C}$ is closed under finite sums, $X$ is an object in $S \mathscr{C}$ and $K$ is a finite simplicial set, then an object $X \otimes K$ exists, defined by

$$
(X \otimes K)_{n}=\sum_{\sigma \in K_{n}} X_{n},
$$

in which the degeneracy and face operators are defined in terms of the corresponding operators of $X$ and $K$. If $\mathscr{C}$ is closed under finite limits, then dually an object $X^{K}$ exists for every finite simplicial set. These have nice universal properties; see Quillen [Q.1].

Therefore given a category $\mathscr{C}$ closed under finite limits and colimits, $S \mathscr{C}$ becomes a simplicial category where the natural functor

$$
\operatorname{Hom}_{S \mathscr{C}}: S \mathscr{C}^{\text {op }} \times S \mathscr{C} \rightarrow S S
$$

is defined by $\operatorname{Hom}_{S \mathscr{C}}(A, B)_{n}=S \mathscr{C}(A \otimes \Delta[n], B)$. If $\mathscr{C}$ is a pointed category, we can also consider the functor $\mathrm{Hom}_{S \mathscr{C}}: \mathscr{C}^{\mathrm{op}} \times S \mathscr{C} \rightarrow S S_{*}$.

In order to have a shorter notation we also use $S=$ Set, $S_{*}=$ Set $_{*}, S_{M}=$ $\operatorname{Set}_{M}, S_{* M}=$ Set $_{* M}$. The corresponding simplicial categories will be denoted by $S S, S S_{*}, S\left(S_{M}\right), S\left(S_{* M}\right)$. We note that, for the functors in: $S_{*} \rightarrow S S_{*}$, in: $S_{* M} \rightarrow S\left(S_{* M}\right)$, and $-\odot M: S S_{*} \rightarrow S\left(S_{* M}\right)$, there are natural isomorphisms

$$
((\text { in } X) \otimes K) \odot M \cong(\text { in }(X \odot M)) \otimes K,
$$

where $X$ is an object in $S_{*}$ and $K$ is a finite simplicial set.

The following result is a particular case of [Q.1, Chapter II, §4, Theorem 4].

Proposition 1. Let $\mathscr{C}$ be a category closed under finite limits and under colimits and having a set $\mathscr{U}$ of small projective generators. Let $S \mathscr{C}$ be the simplicial category of simplicial objects in $\mathscr{C}$. Define a map $f$ in $S \mathscr{C}$ to be a fibration 
(weak equivalence) if $\operatorname{Hom}($ in $P, f$ ) is a fibration (weak equivalence) in $S S$ for each $P$ of $\mathscr{U}$. A map $f$ is a cofibration if $f$ has the left lifting property with respect to the class of trivial fibrations. Then $S \mathscr{C}$ is a closed simplicial model category.

For the case $\mathscr{C}=S_{* M}$ (or $\mathscr{C}=S_{M}$ ), we have that $\mathscr{U}$ has only a single object $S^{0} \otimes M \cong M$. Notice that for a map $f$ of $S\left(S_{* M}\right)$ we have that

$$
\begin{aligned}
& \operatorname{Hom}_{S\left(S_{* M}\right)}\left(\operatorname{in}\left(S^{0} \otimes M\right), f\right) \cong \operatorname{Hom}_{S\left(S_{* M}\right)}\left(\text { in } S^{0} \otimes M, f\right) \\
& \quad \cong \operatorname{Hom}_{S\left(S_{* M}\right)}\left(\Delta[0]^{+} \odot M, f\right) \cong \operatorname{Hom}_{S S_{*}}\left(\Delta[0]^{+}, f\right)=U f .
\end{aligned}
$$

Therefore we have the following closed simplicial model structure:

Definition 1. In the category of simplicial $M$-sets, a map $f$ is said to be a fibration (weak equivalence) if $U f$ is a fibration (weak equivalence) in $S S_{*}$. A map is said to be a cofibration if $f$ has the LLP with respect to any trivial fibration.

Theorem 1. The category $S\left(S_{* M}\right)$ together with the classes of cofibrations, fibrations and weak equivalences defined above has a natural closed simplicial model category structure.

Remark. For the nonpointed case a similar result is obtained for the category $S\left(S_{M}\right)$. The corresponding fibrations and weak equivalences are defined by using the forgetful functor $S\left(S_{M}\right) \rightarrow S S$.

In the category $S S_{*}$, the "tensor" object $X \otimes K$ and the "function" object $X^{K}$ can be defined for any simplicial set $K$. We apply this property to prove the following:

Lemma 1. Let $f$ be a map of $S S_{*}$.

(1) If $f$ is a weak equivalence, then $f \odot M$ is a weak equivalence,

(2) if $f$ is a cofibration, then $f \odot M$ is a cofibration.

Proof. (1) Let $f$ be a weak equivalence in $S S_{*}$; we are going to prove that $f \odot$ in $M$ is a weak equivalence in $S S_{*}$. By [Q.1, Chapter II, Proposition 3.5], it suffices to prove that, for any fibrant object $Y$ of $S S_{*},[f \otimes$ in $M, Y]$ is an isomorphism. This is obtained from the following isomorphisms:

$$
\begin{aligned}
{[f \otimes \text { in } M, Y] } & =\pi_{0} \operatorname{Hom}(f \otimes \text { in } M, Y) \\
& \cong \pi_{0} \operatorname{Hom}(f, \operatorname{Hom}(\operatorname{in} M, Y)) \cong[f, \operatorname{Hom}(\operatorname{in} M, Y)]
\end{aligned}
$$

and the fact that $f$ is a weak equivalence.

The forgetful functor $U: S\left(S_{* M}\right) \rightarrow S S_{*}$ satisfies $U(f \odot M)=f \otimes$ in $M$. Because $U(f \odot M)$ is a weak equivalence, by Definition 1 we also have that $f \odot M$ is a weak equivalence.

(2) Since $-\odot M: S S_{*} \rightarrow S\left(S_{* M}\right)$ is left adjoint to $U: S\left(S_{* M}\right) \rightarrow S S_{*}$, and $U$ preserves weak equivalences and fibrations, we also have that $-\odot M$ preserves cofibrations.

As a consequence of this lemma, we obtain an induced pair of adjoint functors on the localized categories.

Theorem 2. The functions $-\odot M$ and $U$ factot through the homotopy categories in such a way that $-\odot M: \operatorname{Ho}\left(S S_{*}\right) \rightarrow \mathrm{Ho}\left(S\left(S_{* M}\right)\right)$ is left adjoint to 
$U: \mathrm{Ho}\left(S\left(S_{* M}\right)\right) \rightarrow \mathrm{Ho}\left(S S_{*}\right)$. Moreover, $-\otimes M$ preserves cofibration sequences and $U$ preserves fibration sequences.

Remark. If $M$ is a monoid (without zero element), the analogues of the above theorems are similarly obtained. If $M$ is a group, the closed simplicial model category $S\left(S_{M}\right)$ induces a nice homotopy category $\mathrm{Ho}\left(S\left(S_{M}\right)\right)$ to study equivariant homotopy theory.

\section{REALIZATION AND SINGULAR FUNCTORS}

In this section, we analyse the construction of singular and realization functors for the category of simplicial $M$-sets.

Recall that a monoid $M$ can be considered as a category with one object, with morphisms the elements of $M$ and with composition the product in the monoid $M$. Therefore the category of right $M$-sets can be considered as the functor category $\mathrm{Set}^{M^{\mathrm{op}}}$. Thus the category $S\left(S_{M}\right)$ of simplicial $M$-sets is the functor category $\left(\operatorname{Set}^{M^{\mathrm{op}}}\right)^{\Delta^{\mathrm{op}}}$, which is equivalent to the category $\operatorname{Set}^{(M \times \Delta)^{\mathrm{op}}}$.

Given a small category $I$, the functor category $\operatorname{Set}^{{ }^{\text {op }}}$ is also called the category of presheaves on $I$ associated with a functor $X: I^{\text {op }} \rightarrow$ Set . We recall the construction of the category of elements of $X$, denoted by $\int_{I} X$. For more details and properties of this construction, which is often called the Grothendieck construction, we refer the reader to [M-M].

The objects of $\int_{I} X$ are pairs $(i, x)$ where $i$ is an object of $I$ and $x$ is an element of $X(i)$. Its morphisms $\left(i^{\prime}, x^{\prime}\right) \rightarrow(i, x)$ are those morphisms $u: i^{\prime} \rightarrow$ $i$ of $I$ for which $X(u): X(i) \rightarrow X\left(i^{\prime}\right)$ satisfies $X(u) x=x^{\prime}$. This category has a canonical projection functor $\pi_{X}: \int_{I} X \rightarrow I$ defined by $\pi_{X}(i, x)=i$.

The following result is proved in [M-M; Chapter I, Theorem 2]:

Theorem 1. If $\chi: I \rightarrow \mathscr{C}$ is a functor from a small category $I$ to a cocomplete category $\mathscr{C}$, the functor $S_{\chi}$ from $\mathscr{C}$ to Set $^{I^{\text {op }}}$ given by

$$
S_{\chi} C: i \rightarrow \mathscr{C}(\chi(i), C)
$$

has a left adjoint functor $R_{\chi}$ : Set ${ }^{I^{\text {op }}} \rightarrow \mathscr{C}$ defined for each functor $X$ in Set $^{I^{\text {op }}}$ as the colimit

$$
R_{\chi} X=\operatorname{colim}\left(\int_{I} X \underset{\pi_{X}}{\longrightarrow} \underset{\chi}{\longrightarrow} \mathscr{C}\right) .
$$

For the small category $I=M \times \Delta$, the equivalence of categories $S\left(S_{M}\right) \cong$ Set ${ }^{(M \times \Delta)^{\text {op }}}$ carries a functor $X: \Delta^{\text {op }} \rightarrow \operatorname{Set}_{M}$ to a functor $X^{\prime}:(M \times \Delta)^{\text {op }} \rightarrow$ Set . Similarly, for a given category $\mathscr{C}$ and a functor $\chi: \Delta \rightarrow{ }_{M} \mathscr{C}$ one has the corresponding functor $\chi^{\prime}: M \times \Delta \rightarrow \mathscr{C}$. Since $M$ only has one object $*$, the objects of $M \times \Delta$ are of the form $(*,[p])$. However, in the sequel, we just write $[p]$ for the object $(*,[p])$. Recall that a morphism of $M \times \Delta$ is of the form $(m, \varphi):[p] \rightarrow[q]$, where $m$ is an element of $M$ and $\varphi$ is a map of $\Delta$. Observe that $(m, \varphi)=\left(m, \operatorname{id}_{[q]}\right)\left(1_{M}, \varphi\right)=\left(1_{M}, \varphi\right)\left(m, \operatorname{id}_{[p]}\right)$. Sometimes, we just write $m$ for $\left(m, \operatorname{id}_{[q]}\right)$ and $\varphi$ for $\left(1_{M}, \varphi\right)$ if no confusion is possible. For a functor $Y:(M \times \Delta)^{\mathrm{op}} \rightarrow$ Set, we write $Y([p])=Y_{p}, Y(m, \varphi)=(m, \varphi)^{*}$, $y\left(m, \operatorname{id}_{[p]}\right)=Y(m)=m^{*}$ and $Y\left(1_{M}, \varphi\right)=Y(\varphi)=\varphi^{*}$. Similarly for a functor $\chi^{\prime}: M \times \Delta \rightarrow \mathscr{C}$, we write $\chi_{p}^{\prime}$ instead of $\chi^{\prime}([p])$.

Using this notation, we can reformulate the theorem above as follows: 
Theorem 2. If $\chi: \Delta \rightarrow{ }_{M} \mathscr{C}$ is a functor, where $\mathscr{C}$ is a cocomplete category, then the "singular" functor $S_{\chi}$ from $\mathscr{C}$ to $S\left(S_{M}\right)$ defined by

$$
\left(S_{\chi} C\right)_{p}=\mathscr{C}\left(\chi_{p}^{\prime}, \mathscr{C}\right)
$$

has a left adjoint (the "realization" functor) $R_{\chi}: S\left(S_{M}\right) \rightarrow \mathscr{C}$ defined for each $X$ an object in $S\left(S_{M}\right)$ as the colimit

$$
R_{\chi} X=\operatorname{colim}\left(\int_{M \times \Delta} X^{\prime} \underset{\pi_{X^{\prime}}}{\longrightarrow} M \times \Delta \underset{\chi^{\prime}}{\longrightarrow} \mathscr{C}\right) .
$$

In this paper, we will consider functors $\chi: \Delta \rightarrow{ }_{M} \mathscr{C}$, where $\mathscr{C}$ does not have all colimits. For these categories we will analyse those $X$ in $S\left(S_{M}\right)$ for which the colimit $R_{\chi} X$ exists. The following properties of colimits will be useful.

Given a functor $L: J^{\prime} \rightarrow J$ and an object $j$ in $J$, the comma category $j \downarrow L$ has as objects morphisms of the form $u: j \rightarrow L j^{\prime}$. A morphism from $u_{0}: j \rightarrow$ $L j_{0}^{\prime}$ to $u_{1}: j \rightarrow L j_{1}^{\prime}$ is a morphism $v^{\prime}: j_{0}^{\prime} \rightarrow j_{1}^{\prime}$ which satisfies $L\left(v^{\prime}\right) u_{0}=u_{1}$. A category $J$ is called connected if, given any two objects $j_{0}, j_{1}$ in $J$, there is a finite sequence of arrows (both directions possible) joining $j_{0}$ to $j_{1}$.

A functor $L: J^{\prime} \rightarrow J$ is final if for each $j$ in $J$, the comma category $j \downarrow L$ is nonempty and connected. For more details concerning final functors, we refer the reader to [M] and [C-P]. In particular we will use the following:

Proposition 1. If $L: J^{\prime} \rightarrow J$ is final and $F: J \rightarrow \mathscr{C}$ is a functor such that colim $F L$ exists, then colim $F$ exists and the canonical map $\operatorname{colim} F L \rightarrow$ $\operatorname{colim} F$ is an isomorphism.

Definition 1. Given an object $X$ in $S\left(S_{M}\right)$, it is said that $\operatorname{dim} X \leq n$ if for $q \geq n$ and $y \in X_{q}$, there are $p \leq n, x \in X_{p}$ and a surjective map $\varphi:[q] \rightarrow[p]$ such that $y=\varphi^{*} x$.

Denote by $\Delta / n$ the full subcategory of $\Delta$ determined by the objects [0], $\ldots$, [ $n$ ]. Given a functor $X^{\prime}:(M \times \Delta)^{\text {op }} \rightarrow$ Set, one defines the functor $S k_{n} X^{\prime}$ as the composite

$$
(M \times \Delta / n)^{\text {op }} \rightarrow(M \times \Delta)^{\text {op }} \underset{X^{\prime}}{\longrightarrow} \text { Set } .
$$

It is easy to check the existence of a canonical functor $I: \int S k_{n} X^{\prime} \rightarrow \int X^{\prime}$.

Proposition 2. If $X$ is an object in $S\left(S_{M}\right)$ with $\operatorname{dim} X \leq n$, then the functor I: $\int S k_{n} X^{\prime} \rightarrow \int X^{\prime}$ is final.

Proof. Let $([q], y)$ be an object of $\int X^{\prime}$. The condition $\operatorname{dim} X \leq n$ implies that the comma category $([q], y) \downarrow I$ is nonempty. In order to prove that $([q], y) \downarrow I$ is connected it suffices to apply the Eilenberg-Zilber Lemma [G-Z, p. 26].

In this section, we work with the following notions of diagram scheme and diagram. A diagram scheme consists of a set $D_{0}$ of objects and a set $D_{1}$ of arrows together with a source map $s: D_{1} \rightarrow D_{0}$ and a target map $t: D_{1} \rightarrow$ $D_{0}$. For instance, a small category has the structure of a scheme diagram. A morphism $F: D \rightarrow D^{\prime}$ of scheme diagrams consists of a pair of maps $F_{0}: D_{0} \rightarrow$ $D_{0}^{\prime}$ and $F_{1}: D_{1} \rightarrow D_{1}^{\prime}$ such that $s F_{1}=F_{0} s, t F_{1}=F_{0} t$. Let $\mathscr{C}$ be a category. A diagram $F: D \rightarrow \mathscr{C}$ is an operation which assigns to each object of $D$ an object of $\mathscr{C}$ and to each arrow of $D$ a morphism of $\mathscr{C}$. This assignment commutes 
with the source and target operators. The analogues of comma category and connected category are also defined for scheme diagrams and diagrams. Using these notions one has:

Lemma 2. Let $J$ be a small category and let $I: D \rightarrow J$ be a diagram such that $I$ is an inclusion map. Assume that for every $j$ in $J$, there is an associated morphism $u_{j}: j \rightarrow d_{j}$, where $d_{j}$ is an object in $D$. Suppose that these morphisms satisfy:

(i) If $j$ is an object in $D$, then $u_{j}: j \rightarrow d_{j}$ is a morphism in $D$.

(ii) Given a morphism $u: j_{0} \rightarrow j_{1}$ of $J$, the objects $u_{j_{0}}: j_{0} \rightarrow d_{j_{0}}$ and $u_{j_{1}} u: j_{0} \rightarrow d_{j_{1}}$ are in the same connected component of $j_{0} \downarrow I$.

If $F: J \rightarrow \mathscr{C}$ is a functor and colim $F I$ exists, then colim $F$ exists and the canonical map colim $F I \rightarrow \operatorname{colim} F$ is an isomorphism.

Proof. The proof is routine and is left as an exercise.

As an application of the lemma, for some $X$ in $S\left(S_{M}\right)$ with $\operatorname{dim} X \leq$ $n$, we will describe a finite diagram $I: D\left(S k_{n} X^{\prime}\right) \rightarrow \int S k_{n} X^{\prime}$ which satisfies the conditions of Lemma 2 . In this case, in order to prove the existence of $\operatorname{colim}\left(\int X^{\prime} \rightarrow M \times \Delta \stackrel{\chi}{\rightarrow} \mathscr{C}\right)$, it suffices to prove the existence of $\operatorname{colim}\left(D\left(S k_{n} X^{\prime}\right) \rightarrow M \times \Delta \stackrel{\chi^{\prime}}{\rightarrow} \mathscr{C}\right)$. First we introduce some necessary notation.

Let $S_{M} / f f$ be the full subcategory of $S_{M}$ determined by $M$-sets freely generated by finite sets. An object of $S_{M} / f f$ is of the form $\{1, \ldots, n\} \otimes M \cong M \sqcup$ $\cdots \sqcup M$. An element $x$ of $\{1, \ldots, n\} \otimes M$ will be denoted by $x=(i, \alpha)$ where $1 \leq i \leq n$ and $\alpha \in M$. A morphism $u:\{1, \ldots, n\} \otimes M \rightarrow\{1, \ldots, m\} \otimes M$ is determined by a map $\tau_{u}:\{1, \ldots, n\} \rightarrow\{1, \ldots, m\}$ and the values $u\left(1,1_{M}\right)=$ $\left(\tau_{u}(1), u_{1}\right), \ldots$, and $u\left(n, 1_{M}\right)=\left(\tau_{u}(n), u_{n}\right)$, where $u_{1}, \ldots, u_{n} \in M$. If $X$ is an object in $S\left(S_{* M} / f f\right)$, we have that $X_{q}=\left\{1, \ldots, k_{q}\right\} \otimes M$ is generated by the elements $\left(1,1_{M}\right), \ldots,\left(k_{q}, 1_{M}\right)$.

Recall that in the category $\Delta$ we have the canonical maps $\varepsilon_{i}:[p-1] \rightarrow[p]$, $\sigma_{i}:[p+1] \rightarrow[p], 0 \leq i \leq p$. The $i$ th face $\varepsilon_{i}$ is defined by $\varepsilon_{i}(j)=j$ if $j<i$, $\varepsilon_{i}(j)=j+1$ otherwise. The $i$ th degeneracy $\sigma_{i}$ collapses $i+1$ to $i$. For a functor $X: \Delta^{\mathrm{op}} \rightarrow \mathscr{C}$, one usually writes $X\left(\varepsilon_{i}\right)=\varepsilon_{i}^{*}=s_{i}$ and $X\left(\sigma_{i}\right)=\sigma_{i}^{*}=d_{i}$.

Given an object $X$ in $S\left(S_{M} f f\right)$ with $\operatorname{dim} X \leq n$ the diagram $I: D\left(S k_{n} X^{\prime}\right)$ $\rightarrow \int S k_{n} X^{\prime}$, is defined as follows:

If $n=0, D\left(S k_{0} X^{\prime}\right)$ is given by the objects

$$
\left([0],\left(1,1_{M}\right)\right), \ldots,\left([0],\left(k_{0}, 1_{M}\right)\right) .
$$

If $n>0$, for $q=0$ and $1 \leq i \leq k_{0}$, we consider the following objects and arrows in $\int S k_{n} X^{\prime}$ :

$$
\begin{aligned}
& \left([1],\left(\tau_{s_{0}}(i), s_{0} 1_{M}\right)\right) \stackrel{\left(s_{0} 1_{M}, \operatorname{id}_{(11}\right)}{\longrightarrow}\left([1],\left(\tau_{s_{0}}(i), 1_{M}\right)\right) \\
& \quad\left(1_{M}, \sigma_{0}\right) \\
& \quad\left([0],\left(i, 1_{M}\right)\right)
\end{aligned}
$$

For $0<q<n, 1 \leq i \leq k_{q}, 0 \leq j \leq q, 0 \leq l \leq q$, we take the following 
objects and arrows in $\int S k_{n} X^{\prime}$ :

$$
\begin{aligned}
& \left([q+1],\left(\tau_{s_{j}}(i), s_{j} 1_{M}\right)\right) \stackrel{\left(s_{j} 1_{M}, i d_{[q+1]}\right)}{\longrightarrow} \quad\left([q+1],\left(\tau_{s_{j}}(i), 1_{M}\right)\right) \\
& \left(1_{M}, \sigma_{j}\right) \downarrow \\
& \left([q],\left(i, 1_{M}\right)\right) \\
& \left(1_{M}, \varepsilon_{l}\right) \uparrow \\
& \left([q-1],\left(\tau_{d_{l}}(i), d_{l} 1_{M}\right)\right) \stackrel{\left(d_{l} 1_{M}, \operatorname{id}_{[q-1]}\right)}{\longrightarrow} \quad\left([q-1],\left(\tau_{d_{l}}(i), 1_{M}\right)\right)
\end{aligned}
$$

and for $q=n, 1 \leq i \leq k_{n}, 0 \leq l \leq n$, we consider the following objects and arrows:

$$
\begin{gathered}
\left([n],\left(i, 1_{M}\right)\right) \\
\left(1_{M}, \varepsilon_{l}\right) \uparrow \\
\left([n-1],\left(\tau_{d_{l}}(i), d_{l} 1_{M}\right)\right) \stackrel{\left(d_{l} 1_{M}, \operatorname{id}_{[n-1]}\right)}{\longrightarrow}\left([n-1],\left(\tau_{d_{l}}(i), 1_{M}\right)\right)
\end{gathered}
$$

All the objects and arrows given above define a diagram $I: D\left(S k_{n} X^{\prime}\right) \rightarrow$ $\int S k_{n} X^{\prime}$. If $([q],(i, m))$ is an object in $D\left(S k_{n} X^{\prime}\right)$, then we have the map $u=\left(m, \operatorname{id}_{[q]}\right):([q],(i, m)) \rightarrow\left([q],\left(i, 1_{M}\right)\right)$ where $\left([q],\left(i, 1_{M}\right)\right)$ is an object in $D\left(S k_{n} X^{\prime}\right)$. It is easy to check that the family of maps $u$ satisfies the conditions of Lemma 2, so we obtain the following result.

Proposition 2. Let $X$ be an object in $S\left(S_{M} / f f\right)$ with $\operatorname{dim} X \leq n$. If the colimit $\operatorname{colim}\left(D\left(S k_{n} X^{\prime}\right) \rightarrow M \times \Delta \stackrel{\chi^{\prime}}{\rightarrow} \mathscr{C}\right)$ exists, then $\operatorname{colim}\left(\int X^{\prime} \rightarrow M \times \Delta \stackrel{\chi^{\prime}}{\rightarrow} \mathscr{C}\right)$ exists and the two colimits are isomorphic.

As a consequence of Proposition 2, for the case that $\mathscr{C}$ has finite colimits, there is a realization functor $R_{\chi}: S\left(S_{M} / f f\right) / f d \rightarrow \mathscr{C}$ where $S\left(S_{M} / f f\right) / f d$ is the full subcategory determined by objects $X$ in $S\left(S_{M} / f f\right)$ with finite dimension. Next section we will consider the case $\mathscr{C}=$ Pro, where Pro is the category of spaces and proper maps. In this case we have a natural inclusion functor Pro $\rightarrow$ Top into the category Top of spaces and continuous maps. Using the fact that Top has all colimits, we will apply Proposition 2 in order to construct a "proper" realization functor $R_{\chi}: S\left(S_{M} / f f\right) / f d \rightarrow$ Pro.

In this paper, we have to deal with "realization" functors which only are defined on a full subcategory of $S\left(S_{M}\right)$. Then it will be useful to introduce the following notion of partial left adjoint functor.

Definition 1. Let $\mathscr{A}^{\prime}$ be a full subcategory of a category $\mathscr{A}$. We say that $F: \mathscr{A}^{\prime} \rightarrow \mathscr{B}$ is a partial left adjoint to $G: \mathscr{B} \rightarrow \mathscr{A}$ if for any $A$ in $\mathscr{A}^{\prime}$ and $B$ in $\mathscr{B}$, there is a natural isomorphism

$$
\mathscr{B}(F A, B) \cong \mathscr{A}(A, G B) .
$$

For simplicial categories we consider the following notion of simplicial adjunction. 
Definition 2. Let $\mathscr{A}, \mathscr{B}$ be simplicial categories and assume that $F: \mathscr{A} \rightarrow \mathscr{B}$, $G: \mathscr{B} \rightarrow \mathscr{A}$ are functors. We say that $F$ is simplicial left adjoint to $G$, if for any $A$ in $\mathscr{A}$ and $B$ in $\mathscr{B}$, there is a natural simplicial isomorphism

$$
\operatorname{Hom}_{\mathscr{B}}(F A, B) \cong \operatorname{Hom}_{\mathscr{A}}(A, G B) \text {. }
$$

If $\mathscr{A}^{\prime}$ is a full subcategory of $\mathscr{A}$, we say that a functor $F: \mathscr{A}^{\prime} \rightarrow \mathscr{B}$ is partial simplicial left adjoint to $G: \mathscr{B} \rightarrow \mathscr{A}$, if for any $A$ in $\mathscr{A}^{\prime}$ and $B$ in $\mathscr{B}$, there is a natural simplicial isomorphism

$$
\operatorname{Hom}_{\mathscr{B}}(F A, B) \cong \operatorname{Hom}_{\mathscr{A}}(A, G B) .
$$

\section{REALIZATION AND SINGULAR FUNCTORS} FOR PROPER CATEGORIES AND PROCATEGORIES

In this section, we consider the realization and singular functors associated with some covariant functors $\chi: \Delta \rightarrow{ }_{M} C$ and introduce the various notations that will be used later.

1. The standard realization and singular functor. Let $M=\{1\}$ be the monoid having just the unit element. For this monoid it is clear that for any category $C$, ${ }_{M} C=C=C_{M}$. If we consider the standard covariant functor $\chi=$ st: $\Delta \rightarrow$ Top defined by $\operatorname{st}[q]=|\Delta[q]|$, we will obtain the standard realization and singular functors $R_{\mathrm{st}}: S S \rightarrow$ Top, $S_{\mathrm{st}}$ : Top $\rightarrow S S$. The functor $R_{\mathrm{st}}$ is simplicial left adjoint to $S_{\text {st }}$; that is, $\operatorname{Hom}_{\text {Top }}\left(R_{\text {st }} X, Y\right) \cong \operatorname{Hom}_{S S}\left(X, S_{\text {st }} Y\right)$. In this paper the standard realization functor $R_{\mathrm{st}}$ is denoted by $R$ and by ||, and the standard singular functor $S_{\mathrm{st}}$ by $S$.

2. Equivariant realization and singular functors. Given a monoid $M$, it can be provided with the discrete topology and a functor Top $\rightarrow{ }_{M}$ Top can be defined by $X \rightarrow M \times X$. This functor is left adjoint to the forgetful functor ${ }_{M}$ Top $\rightarrow$ Top. If we consider the covariant functor $e=(M \times(-)) \cdot$ st ,

$$
\Delta \underset{\text { st }}{\longrightarrow} \text { Top } \underset{M \times(-)}{\longrightarrow} M \text { Top }
$$

defined by $e[q]=M \times|\Delta[q]|$, we can apply Theorem 3.2 to obtain a realization functor $R_{e}: S\left(S_{M}\right) \rightarrow$ Top $_{M}$ and a singular functor $S_{e}:$ Top $_{M} \rightarrow S\left(S_{M}\right)$.

Given a finite simplicial set $K$ and a object $X$ of Top $_{M}$, there are objects $X \otimes K$ and $X^{K}$ defined by

$$
X \otimes K=X \times|K|, \quad X^{K}=X^{|K|} .
$$

The action of $M$ on $X \otimes K$ is defined by $(x, y) m=(x m, y)$ for $x \in X, y \in$ $|K|$ and $m \in M$, and the action of $M$ on $X^{K}$ is given by $(\varphi m)(y)=(\varphi(y)) m$ for $\varphi \in X^{K}, y \in|K|$ and $m \in M$. As above $R_{e}, S_{e}$ are a pair of simplicial adjoint functors,

$$
\operatorname{Hom}_{\mathrm{Top}_{M}}\left(R_{e} X, Y\right) \cong \operatorname{Hom}_{S\left(S_{M}\right)}\left(X, S_{e} Y\right) .
$$

The equivariant homotopy category is defined to be $\pi_{0}\left(\operatorname{Top}_{M}\right)$. Taking into account the isomorphism above, it follows that

$$
\pi_{0}\left(\mathrm{Top}_{M}\right)\left(R_{e} X, Y\right) \cong \pi_{0}\left(S\left(S_{M}\right)\right)\left(X, S_{e} Y\right) \text {. }
$$


3. Proper realization and singular functors. If we consider the monoid $M=$ $\operatorname{Pro}(\mathbb{N}, \mathbb{N})$, since the identity id: $M \rightarrow \operatorname{Pro}(\mathbb{N}, \mathbb{N})$ is a monoid homomorphism, it follows that $\mathbb{N}$ has the natural structure of a left $M$-set. The functor $-\times|\Delta[q]|:$ Pro $\rightarrow$ Pro induces a left $M$-set structure on $\mathbb{N} \times|\Delta[q]| \cong \bigsqcup_{\mathbb{N}}|\Delta[q]|$ by considering the composite:

$$
M \rightarrow \operatorname{Pro}(\mathbb{N}, \mathbb{N}) \rightarrow \operatorname{Pro}(\mathbb{N} \times|\Delta[q]|, \mathbb{N} \times|\Delta[q]|) .
$$

Therefore there is an induced functor

$$
\chi=p:=\Delta \rightarrow{ }_{M} \text { Pro, } \quad p[q]=\mathbb{N} \times|\Delta[q]| .
$$

The inclusion functor $I:$ Pro $\rightarrow$ Top induces a natural functor ${ }_{M} I:{ }_{M}$ Pro $\rightarrow$ ${ }_{M}$ Top, and we also have the composite:

$$
\chi=c={ }_{M} I \cdot p: \Delta \rightarrow{ }_{M} \text { Pro } \rightarrow{ }_{M} \text { Top . }
$$

Since Top has colimits, applying Theorem 3.2, we obtain the continuous realization functor $R_{c}: S\left(S_{M}\right) \rightarrow$ Top and the continuous singular functor $S_{c}:$ Top $\rightarrow S\left(S_{M}\right)$.

By the exponential law, there is a set isomorphism

$$
\operatorname{Top}(\mathbb{N} \times|\Delta[q]|, X) \cong \operatorname{Top}\left(|\Delta[q]|, X_{c}^{\mathbb{N}}\right)
$$

where the mapping space $X_{c}^{\mathbb{N}}$ has the compact open topology. It is clear that $X_{c}^{\mathrm{N}}$ has the structure of a right $M$-space, therefore we have the following diagram which is commutative up to isomorphism

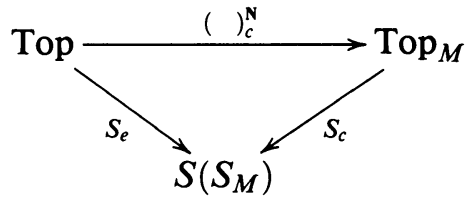

that is, $S_{c} X^{\prime} \cong S_{e}\left(X_{c}^{\mathbb{N}}\right)$.

Recall that for $X$ an object in $S\left(S_{M}\right)$, the functor $R_{c}: S\left(S_{M}\right) \rightarrow$ Top is defined by

$$
R_{c} X=\operatorname{colim}\left(\int X^{\prime} \rightarrow M \times \Delta \stackrel{c^{\prime}}{\longrightarrow} \text { Top }\right) .
$$

If $X$ is an object in $S\left(S_{M} / f f\right) / f d$, by Proposition $3.2 R_{c} X$ is isomorphic to

$$
\operatorname{colim}\left(D\left(S k_{n}\right) X^{\prime} \longrightarrow M \times \Delta \stackrel{c^{\prime}}{\longrightarrow} \text { Top) } .\right.
$$

Since $M \times \Delta \stackrel{c^{\prime}}{\rightarrow}$ Top factor as $M \times \Delta \stackrel{p^{\prime}}{\rightarrow}$ Pro $\stackrel{I}{\rightarrow}$ Top and using the fact that for any object $(p[q],(i, m))$ in $D\left(S k_{n} X^{\prime}\right)$ the continuous map $c^{\prime}[q]=$ $\mathbb{N} \times|\Delta[q]| \rightarrow R_{c} X$ is proper, it follows that $\operatorname{colim}\left(D\left(S k_{n} X^{\prime}\right) \rightarrow M \times \Delta \stackrel{p^{\prime}}{\rightarrow}\right.$ Pro $)$ exists. Applying again Proposition 3.2, one has the $\operatorname{colim}\left(\int X^{\prime} \rightarrow M \times \Delta \stackrel{p^{\prime}}{\rightarrow}\right.$ Pro) exists. Therefore, for any $X$ an object in $S\left(S_{M} / f f\right) / f d$ we can define $R_{p}: S\left(S_{M} f f\right) / f d \rightarrow$ Pro by

$$
R_{p} X=\operatorname{colim}\left(\int X^{\prime} \rightarrow M \times \Delta \underset{p^{\prime}}{\longrightarrow} \text { Pro }\right) .
$$

On the other hand, observe that the set $X_{p}^{\mathbb{N}}=\operatorname{Pro}(\mathbb{N}, X)$ is bijective to the subset $\left\{f \in \widehat{X}_{c}^{\widehat{N}} \mid f^{-1} \infty=\infty\right\}$. We will consider on $X_{p}^{\mathbb{N}}$ the relative topology 
induced by the compact open topology of the space $\widehat{X}_{c}^{\widehat{N}}$. It is easy to check that we have a natural set-isomorphism

$$
\operatorname{Pro}(\mathbb{N} \times|\Delta p[q]|, X) \cong \operatorname{Top}\left(|\Delta[q]|, X_{p}^{\mathbb{N}}\right) .
$$

These sets have also a natural structure as right $M$-sets $(M=\operatorname{Pro}(\mathbb{N}, \mathbb{N}))$, and the isomorphism above becomes an $M$-set isomorphism. Therefore we have the following diagram of functors which is commutative up to natural isomorphism:

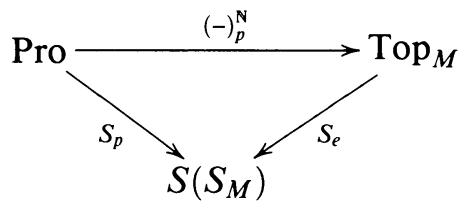

The pair of adjoint functors $R_{c}: S\left(S_{M}\right) \rightarrow$ Top, $S_{c}$ : Top $\rightarrow S\left(S_{M}\right)$ possesses the following properties (a)-(c).

(a) $S_{c}$ preserves "function" functors: for a finite simplicial set $K$ and an object $X$ of Top $_{M}$, we have

$$
S_{c}\left(X^{K}\right) \cong S_{e}\left(\left(X^{|K|}\right)_{c}^{\mathbb{N}}\right) \cong S_{e}\left(\left(X_{c}^{\mathbb{N}}\right)^{|K|}\right) \cong\left(S_{e} X_{c}^{\mathbb{N}}\right)^{K} \cong\left(S_{c} X\right)^{K} .
$$

(b) $R_{c}$ preserves "tensor" functors. Let $X$ be an object of $S\left(S_{M}\right)$ and let $Y$ be a topological space. Then

$$
\begin{aligned}
\operatorname{Top}\left(R_{c} X \otimes K, Y\right) & \cong \operatorname{Top}\left(R_{c} X, Y^{K}\right) \cong S\left(S_{M}\right)\left(X, S_{c}\left(Y^{K}\right)\right) \\
& \cong S\left(S_{M}\right)\left(X,\left(S_{c} Y\right)^{K}\right) \cong S\left(S_{M}\right)\left(X \otimes K, S_{c} Y\right) \\
& \cong \operatorname{Top}\left(R_{c}(X \otimes K), Y\right) .
\end{aligned}
$$

By the Yoneda lemma, it follows that $R_{c} X \otimes K \cong R_{c}(X \otimes K)$. This implies that $R_{c}$ is simplicial left adjoint to $S_{c}$; that is,

$$
\operatorname{Hom}_{\mathrm{Top}}\left(R_{c} X, Y\right) \cong \operatorname{Hom}_{S\left(S_{M}\right)}\left(X, S_{c} Y\right) \text {. }
$$

(c) $R_{p}: S\left(S_{M} / f f\right) / f d \rightarrow$ Pro preserves "tensor" functors. This follows because $R_{p}=R_{c}$ on the full subcategory $S\left(S_{M} / f f\right) / f d$. Observe that the "tensor" functor of Top (see $\S 1$, Example (2)) induces a "tensor" functor on the subcategory Pro of spaces and proper maps. Notice that we only consider "tensor" functors associated with finite simplicial sets. Using this tensor produce one can define a functor $\mathrm{Hom}_{\mathrm{Pro}}$ : Pro $^{\text {op }} \times$ Pro $\rightarrow S S$ by

$$
\operatorname{Hom}_{\mathrm{Pro}}(X, Y)_{q}=\operatorname{Pro}(X \otimes \Delta[q], Y) \text {. }
$$

In this way Pro becomes a simplicial category, and the standard proper homotopy category is defined to be $\pi_{0}$ (Pro) .

Because $\left.R_{p}: S\left(S_{M} / f f\right) / f d\right) \rightarrow$ Pro preserves "tensor" functors, we have the following isomorphisms:

$$
\begin{aligned}
\operatorname{Hom}_{\mathrm{Pro}}\left(R_{p} X, Y\right)_{q} & \cong \operatorname{Pro}\left(R_{p} X \otimes \Delta[q], Y\right) \cong \operatorname{Pro}\left(R_{p}(X \otimes \Delta[q]), Y\right) \\
& \cong S\left(S_{M}\right)\left(X \otimes \Delta[q], S_{p} Y\right) \cong \operatorname{Hom}_{S\left(S_{M}\right)}\left(X, S_{p} Y\right)_{q} .
\end{aligned}
$$

This implies that we have a simplicial isomorphism

$$
\operatorname{Hom}_{\text {Pro }}\left(R_{p} X, Y\right) \cong \operatorname{Hom}_{S\left(S_{M}\right)}\left(X, S_{p} Y\right)
$$

and $R_{p}: S\left(S_{M} / f f\right) / f d \rightarrow$ Pro is a partial simplicial left adjoint functor for $S_{p}$ : Pro $\rightarrow S\left(S_{M}\right)$. The functors $R_{p}$ and $S_{p}$ induce the following adjointness 
on the categories $\pi_{0}$ (Pro) and $\pi_{0}\left(S\left(S_{M}\right)\right)$. If $X$ is an object of $S\left(S_{M} / f f\right) / f d$ and $Y$ an object of Pro, then

$$
\pi_{0}(\mathrm{Pro})\left(R_{p} X, Y\right) \cong \pi_{0}\left(S\left(S_{M}\right)\right)\left(X, S_{p} Y\right) .
$$

The last properties give the following results:

Theorem 1. The proper realization functor $R_{p}: S\left(S_{M} / f f\right) f d \rightarrow$ Pro is simplicial partial left adjoint to the proper singular functor $S_{p}$ : Pro $\rightarrow S\left(S_{M}\right)$.

Theorem 2. If $X$ is a cofibrant object of $S\left(S_{M} / f f\right) / f d$ and $Y$ an object of Pro, then

$$
\pi_{0}(\operatorname{Pro})\left(R_{p} X, Y\right) \cong \mathrm{Ho}\left(S\left(S_{M}\right)\right)\left(X, S_{p} Y\right)
$$

Proof. Let $U: S\left(S_{M}\right) \rightarrow S S$ denote the forgetful functor. Notice that

$$
U\left(S_{p} Y\right) \cong U S_{e}\left(Y_{p}^{\mathbb{N}}\right)=S\left(Y_{p}^{\mathbb{N}}\right)
$$

is a fibrant object of $S S$. By the definition of fibration in $S\left(S_{M}\right)$, see Definition 2.1 , it follows that $S_{p} Y$ is fibrant in $S\left(S_{M}\right)$. Therefore we have

$$
\begin{aligned}
\pi_{0}(\operatorname{Pro})\left(R_{p} X, Y\right) & =\pi_{0} \operatorname{Hom}_{\text {Pro }}\left(R_{p} X, Y\right) \cong \pi_{0} \operatorname{Hom}_{S\left(S_{M}\right)}\left(X, S_{p} Y\right) \\
& \cong \operatorname{Ho}\left(S\left(S_{M}\right)\right)\left(X, S_{p} Y\right) .
\end{aligned}
$$

The last isomorphism follows from the fact that $X$ is cofibrant and $S_{p} Y$ is fibrant.

4. Realization and singular functors for pro-spaces. Let $C$ be a category with countable sums (coproducts). Using the sum of $C$, we can define a functor $c: C \rightarrow$ pro $C$ as follows. If $X$ is an object of $C, c X: \mathbb{N} \rightarrow C$ is defined by

$$
(c X)_{i}=\bigsqcup_{j \geq i} X
$$

where $\mathbb{N}$ is consider in this case with its left filtering category structure. The standard "inclusions" of the coproduct define the natural map $(c X)_{i+1} \rightarrow(c X)_{i}$. Recall that the objects of the category (pro $C, C$ ) are promorphisms of the form $Y \rightarrow B$, where $Y: I \rightarrow C$ is an object in pro $C$ and $B: 1=\{0\} \rightarrow C$ is a constant object. To determine a promorphism $Y \rightarrow B$ it suffices to give a map $\varphi: 1 \rightarrow I$ and a morphism $f_{\varphi(0)}: Y_{\varphi(0)} \rightarrow B_{0}$. Since $c X: \mathbb{N} \rightarrow C$ is an object in pro $C$ and $(c X)_{0}$ is a constant object, the maps $\varphi: 1 \rightarrow \mathbb{N}, \varphi(0)=0$, and id: $(c X)_{\varphi(0)} \rightarrow(c X)_{0}$ determine a promorphism $c_{g} X: c X \rightarrow(c X)_{0}$; that is, an object in (pro $C, C$ ). This defines a global (or argumented) functor $c_{g}: C \rightarrow($ pro $C, C)$. If it is necessary to distinguish the two functors we shall use the notation $c_{\infty}: C \rightarrow$ pro $C$ and $c_{g}: C \rightarrow($ pro $C, C)$ otherwise we just write $c$.

Consider the following functors.

(a) The functor $\chi_{\infty}: \Delta \rightarrow M_{\infty}($ Pro $S S)$.

The functor $c: S S \rightarrow$ pro $S S$ gives an object $c \Delta[0]$ in pro $S S$, and we can consider the monoid $M_{\infty}=\operatorname{pro} S S(c \Delta[0], c \Delta[0])$ which is isomorphic to $\operatorname{Pro}_{\infty}(\mathbb{N}, \mathbb{N})$. As in subsection $3, c \Delta[0]$ has a natural structure as a left $M_{\infty}$-object. The functor $-\otimes \Delta[q]:$ pro $S S \rightarrow$ pro $S S$ induces left $M_{\infty}$-object structures on $c \Delta[0] \otimes \Delta[q] \cong c \Delta[q]$, so there is a functor $\chi_{\infty}: \Delta \rightarrow M_{\infty}($ pro $S S)$, $\left(\chi_{\infty}\right)_{q}=c \Delta[q]$. Now by Theorem 3.2, we obtain a realization singular functor 
$R_{\chi_{\infty}}: S\left(S_{M_{\infty}}\right) \rightarrow$ pro $S S$ which is simplicial left adjoint to the corresponding singular functor $S_{\chi_{\infty}}:$ pro $S S \rightarrow S\left(S_{M_{\infty}}\right)$.

(b) The global functor $\chi_{g}: \Delta \rightarrow{ }_{M}($ pro $S S, S S)$.

Using the global version of the $c$ functor, $c_{g}: S S \rightarrow($ pro $S S, S S)$, and the monoid $M=(\operatorname{pro} S S, S S)\left(c_{g} \Delta[0], c_{g} \Delta[0]\right)$ which is isomorphic to $\operatorname{Pro}(\mathbb{N}, \mathbb{N})$, we have an induced functor $\chi_{g}: \Delta \rightarrow{ }_{M}($ pro $S S, S S)$. Associated with the functor $\chi_{g}$, there are a realization functor $R_{\chi_{g}}: S\left(S_{M}\right) \rightarrow($ pro $S S, S S)$ and a singular functor $S_{\chi_{8}}:($ pro $S S, S S) \rightarrow S\left(S_{M}\right)$ that induce a simplicial adjunction isomorphism

$$
\operatorname{Hom}_{(\operatorname{pro} S S, S S)}\left(R_{\chi_{g}} X, Y\right) \cong \operatorname{Hom}_{S\left(S_{M}\right)}\left(X, S_{\chi_{g}} Y\right) .
$$

(c) The functor $\chi_{\infty}^{*}: \Delta \rightarrow M_{\infty}^{*}\left(\right.$ pro $\left.S S_{*}\right)$.

Recall that for a simplicial set $K, K \sqcup \Delta[0]$ is denoted by $K^{+}$. Using the functor $c: S S_{*} \rightarrow$ pro $S S_{*}$, we get the object $c \Delta[0]$ and we can consider the monoid $M_{\infty}^{*}=\operatorname{pro} S S_{*}\left(c \Delta[0]^{+}, c \Delta[0]^{+}\right)$which is isomorphic to $\operatorname{Top}_{*}^{\infty}(\widehat{\mathbb{N}}, \widehat{\mathbb{N}})$. As a consequence of Theorem 3.2, we also have a natural adjunction isomorphism

$$
\operatorname{Hom}_{\text {pro } S S_{*}}\left(R_{\chi_{\infty}^{*}} X, Y\right) \cong \operatorname{Hom}_{S\left(S_{M_{\infty}^{*}}\right)}\left(X, S_{\chi_{\infty}^{*}} Y\right) \text {. }
$$

(d) The functor $\chi_{g}^{*}: \Delta \rightarrow M_{g}^{*}\left(\right.$ pro $\left.S S_{*}, S S_{*}\right)$.

The monoid $M_{g}^{*}=\left(\operatorname{pro} S S_{*}, S S_{*}\right)\left(c_{g} \Delta[0]^{+},\left(c_{g} \Delta[0]^{+}\right)\right.$is isomorphic to $\operatorname{Top}_{*}(\widehat{\mathbb{N}}, \widehat{\mathbb{N}})$. As in the cases above, we have a functor

$$
\chi_{g}^{*}: \Delta \rightarrow{ }_{M_{g}^{*}}\left(\operatorname{pro} S S_{*}, S S_{*}\right)
$$

and a simplicial isomorphism

$$
\operatorname{Hom}_{\left(\operatorname{pro} S S_{*}, S S_{*}\right)}\left(R_{\chi_{g}^{*}} X, Y\right) \cong \operatorname{Hom}_{S\left(S_{M_{g}^{*}}\right)}\left(X, S_{\chi_{g}^{*}} Y\right) .
$$

\section{BROWN's $\mathscr{P}$ FUNCTOR AND THE SINGULAR FUNCTOR pro $S S \rightarrow S\left(S_{M}\right)$}

In 1975, E. M. Brown [Br.1] gave a definition of a proper fundamental group ${ }^{B} \pi_{1}^{\infty}(X)$ of a $\sigma$-compact space $X$ with a base ray. He also defined a functor $\overline{\mathscr{P}}:$ tow $\mathrm{Gps} \rightarrow \mathrm{Gps}$ that gives the relation between the tower of fundamental groups, $\pi_{1} \varepsilon X$, of a tower of neighbourhoods of $X$ at infinity and the proper fundamental group. This relation is given by $\overline{\mathscr{P}} \pi_{1} \varepsilon X \cong{ }^{B} \pi_{1}^{\infty}(X)$. In this section we extend this definition to other categories and study the relation with the singular functor pro $S S \rightarrow S\left(S_{M}\right)$.

Let $C$ denote one of the following categories:

$$
\begin{aligned}
& \text { Set }=\text { (sets) }, \\
& \text { Set } \left._{*}=\text { (pointed sets }\right), \\
& \text { Gps }=\text { (graphs }) \\
& \mathrm{Ab}=\text { (abelian groups) } .
\end{aligned}
$$

The small projective generators of these (algebraic) categories will be denoted by $*, S^{0}, \mathbb{Z}, \mathbb{Z}_{a}$, respectively.

Since $C$ has sums, we have the functor $c: C \rightarrow$ pro $C$ defined by $c X: \mathbb{N} \rightarrow$ $C,(c X)_{i}=\bigsqcup_{j \geq i} X$. Sometimes, we will also consider the global (or augmented) version $c: C \rightarrow($ pro $C, C)$.

Let $G$ denote the small projective generator of $C$ and let $\mathscr{P}_{c} G$ denote the endomorphism set

$$
\mathscr{P} c G=\operatorname{pro} C(c G, c G) .
$$


If $C=$ Set and $G=*, \mathscr{P}_{C *}$ has a monoid structure. Notice that $\mathscr{P}_{\mathcal{C} *} \cong$ $\operatorname{pro} S S(c \Delta[0], c \Delta[0]) \cong \operatorname{Pro}(\mathbb{N}, \mathbb{N})$. In the pointed case, $C=\operatorname{Set}_{*}, G=S^{0}$, the endomorphism set admits the structure of a 0 -monoid, see $\S 2$, and we have that $\mathscr{P} c S^{0} \cong \operatorname{pro} S S_{*}\left(c \Delta[0]^{+}, c \Delta[0]^{+}\right) \cong \operatorname{Top}_{*}^{\infty}(\widehat{\mathbb{N}}, \widehat{\mathbb{N}})$.

If $C=\mathrm{Gps}, G=\mathbb{Z}$, the endomorphism set has a natural near-ring structure, see [Mel, Pilz]. Finally for $C=\mathrm{Ab}, G=\mathbb{Z}_{a}$, the endomorphism set $\mathscr{P} c \mathbb{Z}_{a}$ becomes a ring isomorphic to the ring of locally finite matrices modulo the ideal of finite matrices, see [F-W.1, F-W.2].

Let $C_{\mathscr{P}_{c G}}$ denote one of the following categories:

If $C=$ Set, $G=*$, then $\operatorname{Set}_{\mathscr{P}_{C *}}$ is the category of right $\mathscr{P}_{C * \text {-sets. If }}$ $C=\operatorname{Set}_{*}, G=S^{0}$, then $\operatorname{Set}_{*} \mathscr{P}_{c} S^{0}$ is the category of right $\mathscr{P}_{c} S^{0}$-pointed sets.

If $C=\mathrm{Grp}, G=\mathbb{Z}$, then $\mathrm{Grp}_{\mathscr{P}} \mathbb{Z}$ is the category of right $\mathscr{P} c \mathbb{Z}$-groups. This category is also known as the category of right near modules over the near-ring $\mathscr{P} c \mathbb{Z}$, see [Mel, Pilz].

If $C=\mathrm{Ab}, G=\mathbb{Z}_{a}$, then $\mathrm{Ab}_{\mathscr{P}_{c} \mathbb{Z}_{a}}$ is the category of right $\mathscr{P}_{c} \mathbb{Z}_{a}$-abelian groups that is usually called the category of right $\mathscr{P} c \mathbb{Z}_{a}$-modules.

If we consider the global (or augmented) functor $c=c_{g}: C \rightarrow($ pro $C, C)$, we will get the endomorphism set $\mathscr{P}_{g} c_{g} G=($ pro $C, C)\left(c_{g} G, c_{g} G\right)$ that will also be denoted by $\mathscr{P}_{g} c G$ and the corresponding category $C_{\mathscr{P}_{g} c G}$.

Given an object $X$ of pro $C$, it is easy to check that pro $C(c G, X)$ is an object of $C_{\mathscr{P} c G}$. Therefore we have a functor

$$
\mathscr{P}: \text { pro } C \rightarrow C_{\mathscr{P} C G}
$$

defined by $\mathscr{P} X=$ pro $C(c G, X)$.

The full subcategory of pro $C$ determined by objects indexed by natural numbers is usually denoted by tow $C$. We say that an object $X$ of pro $C$ is nitely generated if there is an effective epimorphism $Q \rightarrow X$ where $Q$ is a finite sum of copies of $c G$.

We summarize some properties of the $\mathscr{P}$ functors in the following results, see [He.1].

Theorem 1. The functor $\mathscr{P}:$ pro $C \rightarrow C_{\mathscr{P} c G}$ satisfies:

(i) the restriction $\mathscr{P}:$ tow $C \rightarrow C_{\mathscr{P} c G}$ is faithful;

(ii) the restriction $\mathscr{P}:$ tow $C / f g \rightarrow C_{\mathscr{P}_{c G}}$ is also full, where tow $C / f g$ is the full subcategory of tow $C$ of finitely generated towers.

Theorem 2. The functor $\mathscr{P}:$ pro $C \rightarrow C_{\mathscr{P} c G}$ has a left adjoint functor $\mathscr{L}: C_{\mathscr{P}_{c} G}$ $\rightarrow$ pro $C$.

Remark. There are similar results for the category (pro $C, C$ ) of global proobjects in $C$ and the category $C_{\mathscr{P}_{g} c G}$.

Since the forgetful functor $U: C_{\mathscr{P} c G} \rightarrow C$ has a left adjoint function $-\odot \mathscr{P}_{C} G: C \rightarrow C_{\mathscr{P} c G}$, we have the pairs of adjoint functors

$$
\operatorname{pro} C \underset{\mathscr{P}}{\stackrel{\mathscr{L}}{\leftrightarrows}} C_{\mathscr{P} c G} \underset{U}{\stackrel{-\mathscr{\odot P}_{c}}{\rightleftarrows}} C
$$

and the composites $\overline{\mathscr{L}}=\mathscr{L}\left(-\odot \mathscr{P}_{c} G\right), \overline{\mathscr{P}}=U \mathscr{P}$ give a new pair of adjoint functors

$$
\operatorname{pro} C \underset{\overline{\mathscr{P}}}{\stackrel{\overline{\mathscr{I}}}{\longrightarrow}} C \text {. }
$$


Notice that $\overline{\mathscr{P}}:$ pro Gps $\rightarrow$ Gps is the functor defined by Brown and denoted in his paper $[\mathrm{Br} .1]$ by $\mathscr{P}$.

Remark. It is easy to check that $\overline{\mathscr{L}} G=\mathscr{L}\left(G \odot \mathscr{P}_{c} G\right) \cong \mathscr{L}\left(\mathscr{P}_{c} G\right)=c G$ and $\overline{\mathscr{L}} f=c f$ for any morphism $f: G \rightarrow G$. Therefore $\overline{\mathscr{L}}=c$ on the full subcategory of $C$ obtained from $G$ by considering finite colimits of copies of $G$.

Given a left filtering small category $I$, the equivalence of categories $\left(C^{I}\right)^{\Delta} \simeq$ $\left(C^{\Delta}\right)^{I}$ induces a natural functor $F:$ pro $S C \rightarrow S$ pro $C$ defined by $(F X)_{q}(i)=$ $(X(i))_{q}$, where $X$ is an object in pro $S C$. On the other hand the functors $\mathscr{L}: C_{\mathscr{P} c G} \rightarrow$ pro $C$ and $\mathscr{P}:$ pro $C \rightarrow C_{\mathscr{P}_{c G}}$ induce functors $S \mathscr{L}: S C_{\mathscr{P}_{c} G} \rightarrow$ $S$ pro $C$ and $S \mathscr{P}: S$ pro $C \rightarrow S C_{\mathscr{P} c G}$. Next we analyse the relation between the $\mathscr{P}$ functor and the singular functor $S_{\chi_{\infty}}$ : pro $S C \rightarrow S\left(C_{\mathscr{P}_{C G}}\right)$ defined in $\S 4.4$.

The relation between these functors is given in the following:

Theorem 3. The following diagram is commutative up to natural isomorphism:

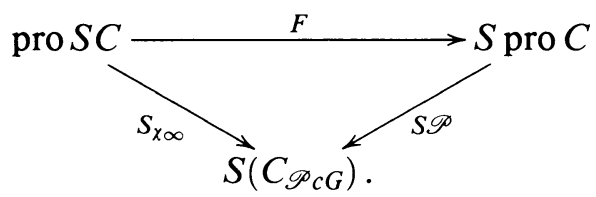

Proof. We are going to use the fact that the functor $c: C \rightarrow$ pro $C$ agrees with $\overline{\mathscr{L}}$ in some cases (see the Remark above). We also consider several functors of the form in: $\mathscr{C} \rightarrow S \mathscr{C}$, where $(\text { in } X)_{q}=X$ and the face and degeneracy operators are equal to the identity of $X$. We have the following isomorphisms:

$$
\begin{aligned}
\left(S_{\chi_{\infty}} X\right)_{q} & =\operatorname{pro} S C\left(\chi_{\infty}[q], X\right) \\
& \cong \operatorname{pro} S C(c(\operatorname{in} G \otimes \Delta[q]), X) \\
& \cong \operatorname{pro} C C(c \operatorname{in} G \otimes \Delta[q], X) \\
(1) & \cong S \operatorname{pro} C(F(c \text { in } G \otimes \Delta[q]), F X) \\
& \cong S \operatorname{pro} C(F c \text { in } G \otimes \Delta[q], F X) \\
& \cong S \operatorname{pro} C(\operatorname{in} c G \otimes \Delta[q], F X) \\
& \cong S \operatorname{pro} C(\operatorname{in} \mathscr{L} G \otimes \Delta[q], F X) \\
& \cong S \operatorname{pro} C(\operatorname{in} \mathscr{L}(G \odot \mathscr{P} c G) \otimes \Delta[q], F X) \\
& \cong S \operatorname{pro} C(S \mathscr{L} \operatorname{in}(G \odot \mathscr{P} c G) \otimes \Delta[q], F X) \\
& \cong S \operatorname{pro} C(S \mathscr{L}(\operatorname{in}(G \odot \mathscr{P} c G) \otimes \Delta[q], F X) \\
& \cong S\left(S_{\mathscr{P} c G}\right)(\operatorname{in}(G \odot \mathscr{P} c G) \otimes \Delta[q], S \mathscr{P} F X) \\
& \cong(S \mathscr{P} F X)_{q} .
\end{aligned}
$$

The isomorphism (1) follows from the fact that $c$ in $G \otimes \Delta[q]$ is a finite-dimensional pro-object. Let $S_{\leq q} \mathscr{C}$ denote the category of $q$-truncated simplicial objects in $\mathscr{C}$; that is, functors $(\Delta / q)^{\text {op }} \rightarrow \mathscr{C}$ where $\Delta / q$ is the full subcategory of $\Delta$ determined by the objects $[0],[1], \ldots,[q]$. It is not hard to check that

$$
\begin{aligned}
\operatorname{pro} S C(c \text { in } G \otimes \Delta[q], X) & \cong \text { pro } S_{\leq q} C(c \text { in } G \otimes \Delta[q], X) \\
(2) & \cong S_{\leq q} \text { pro } C(F(c \text { in } G \otimes \Delta[q]), F X) \\
& \cong S \text { pro } C(F(c \text { in } G \otimes \Delta[q]), F X) .
\end{aligned}
$$


The isomorphism (2) is a consequence of the theorem of C. V. Meyer [Mey] that says that $\operatorname{pro}\left(C^{D}\right)$ is equivalent to $(\operatorname{pro} C)^{D}$ if $D$ is a finite category and $C$ has finite limits.

Remark. As a consequences of Theorem 3, we observe that the singular functor $S_{\chi_{\infty}}$ is calculated dimensionwise by the $\mathscr{P}$ functor. In this way, the functor $S_{\chi_{\infty}}$ can be considered as an extension of the functor $\mathscr{P}:$ pro $C \rightarrow C_{\mathscr{P} c G}$. For this reason, in the sequel, the functors $S_{\chi_{\infty}}, S_{\chi_{g}}, S_{\chi_{\infty}^{*}}, S_{\chi_{8}^{*}}$ will be denoted by $\mathscr{P}$ and the corresponding realization functors $R_{\chi_{\infty}}, R_{\chi_{g}}, R_{\chi_{\infty}^{*}}, R_{\chi_{g}^{*}}$ by $\mathscr{L}$.

The forgetful functors Set $_{*} \rightarrow$ Set, Gps $\rightarrow$ Set $_{*}$ and $\mathrm{Ab} \rightarrow$ Gps have left adjoint functors denoted by

$$
()^{+}: \text {Set } \rightarrow \text { Set }_{*}, \quad f: \text { Set }_{*} \rightarrow \text { Gps, } \quad \text { ab: Gps } \rightarrow \mathrm{Ab} .
$$

We consider the induced functors

$$
\begin{gathered}
\operatorname{pro}()^{+}: \text {proSet } \rightarrow \text { pro Set }_{*}, \\
\text { pro } f: \text { proSet } \\
\text { pro }(\mathrm{ab}): \text { proGps } \mathrm{Gps},
\end{gathered}
$$

and the induced monoid homomorphisms

$$
\operatorname{proSet}\left(c_{*}, c_{*}\right) \rightarrow \operatorname{proSet}_{*}\left(c S^{0}, c S^{0}\right) \rightarrow \operatorname{pro} \operatorname{Grp}(c \mathbb{Z}, c \mathbb{Z}) \rightarrow \operatorname{pro} \mathrm{Ab}\left(c \mathbb{Z}_{a}, c \mathbb{Z}_{a}\right) \text {. }
$$

Using the isomorphism $\operatorname{Pro}_{\infty}(\mathbb{N}, \mathbb{N}) \cong \operatorname{pro} S\left(c_{*}, c_{*}\right)$, the proper map sh: $\mathbb{N}$ $\rightarrow \mathbb{N}, \operatorname{sh}(i)=i+1, i \in \mathbb{N}$, defines an element of pro $S\left(c_{*}, c_{*}\right)$. The monoid homomorphisms above determine new canonical elements in the other monoids. Any one of these elements will be denoted by sh and will be called the shift operator.

For $C$ any of the categories with which we are working, we define a functor $F_{\mathrm{sh}}: C_{\mathscr{P} c G} \rightarrow C$ by

$$
F_{\mathrm{sh}} X=\{x \in X \mid x \mathrm{sh}=x\} .
$$

It is easy to check the functor diagram

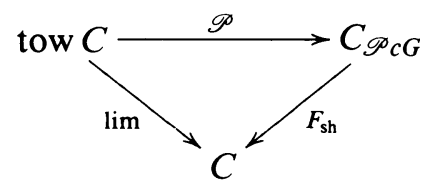

is commutative up to natural isomorphism, where lim is the standard inverse limit. We can also prove the following result.

Theorem 4. The diagram

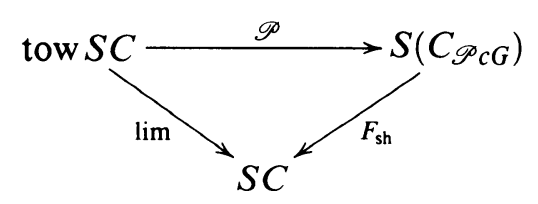

is commutative up to natural isomorphism. 
Proof. By Theorem 3, $\mathscr{P}$ is isomorphic to $S \mathscr{P} F$. It is clear that $S \lim \cong$ $S F_{\text {sh }} S \mathscr{P}$. Since the diagram

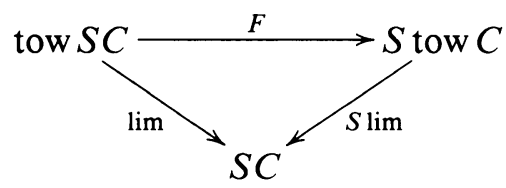

is commutative, we have that

$$
F_{\mathrm{sh}} \mathscr{P} \cong F_{\mathrm{sh}} S \mathscr{P} F \cong S F_{\mathrm{sh}} S \mathscr{P} F \cong S \lim F \cong \lim .
$$

Remark. The functors lim: pro $S C \rightarrow S C, \mathscr{P}: \operatorname{pro} S C \rightarrow S\left(C_{\mathscr{P}_{c} G}\right)$, and $F_{\mathrm{sh}}$ : $S\left(C_{\mathscr{P} c G}\right) \rightarrow S C$ have left adjoint functors.

\section{DeRIVED FUnCtors of $\mathscr{L}$ AND $\mathscr{P}$}

In this section we analyse the properties of the pair of adjoint functors $\mathscr{L}=$ $R_{\chi_{\infty}^{*}}: S\left(S_{* \mathscr{P} c S^{0}}\right) \rightarrow$ pro $S S_{*}, \mathscr{P}=S_{\chi_{\infty}^{*}}:$ pro $S S_{*} \rightarrow S\left(S_{* \mathscr{P} c S^{0}}\right)$ with respect to the closed model structures of these categories. In the category pro $S S_{*}$ we consider the structure given by Edwards and Hastings [E-H], and the category $S\left(S_{* \mathscr{D} C S^{0}}\right)$ is provided with the structure given in $\S 2$.

Recall that if $\mathscr{A}^{\prime}$ is a full subcategory of $\mathscr{A}$, we say that a functor $F: \mathscr{A}^{\prime} \rightarrow$ $\mathscr{B}$ is a partial left adjoint to the functor $G: \mathscr{B} \rightarrow \mathscr{A}$ if for any $A$ of $\mathscr{A}^{\prime}$ and $B$ of $\mathscr{B}$ there is a natural isomorphism $\mathscr{B}(F A, B) \cong \mathscr{A}(A, G B)$. If $\mathscr{A}, \mathscr{B}$ are simplicial categories and this isomorphism extends to a simplicial isomorphism $\operatorname{Hom}_{\mathscr{B}}(F A, B) \cong \operatorname{Hom}_{\mathscr{A}}(A, G B)$, it is said that $F$ is a partial simplicial left adjoint functor to $G$.

Lemma 1. The restriction of the functor $c: S S_{*} \rightarrow \operatorname{pro} S S_{*}$ to the full subcategory of finite simplicial sets is a partial simplicial left adjoint functor to $\overline{\mathscr{P}}=U \mathscr{P}:$ pro $S S_{*} \rightarrow S S_{*}$.

Proof. For a finite simplicial set $X$, we have the isomorphisms

$$
\begin{aligned}
& \operatorname{Hom}_{\text {pro } S S_{*}}(c X, Y) \cong \operatorname{Hom}_{\text {pro } S S_{*}}\left(c \Delta[0]^{+} \otimes X, Y\right) \\
& \quad \cong \operatorname{Hom}_{S S_{*}}\left(X, \operatorname{Hom}_{\text {pro } S S_{*}}\left(c \Delta[0]^{+}, Y\right)\right) \cong \operatorname{Hom}_{S S_{*}}(X, \overline{\mathscr{P}} Y) .
\end{aligned}
$$

Lemma 2. The functor $\mathscr{P}: \operatorname{pro} S S_{*} \rightarrow S\left(S_{* \mathscr{D} C S^{0}}\right)$ satisfies the following conditions:

(1) If $p: E \rightarrow B$ is a fibration in pro $S S_{*}$ in the Edwards-Hastings sense, then $\mathscr{P} p$ is a fibration in $S\left(S_{* \mathscr{P} c S^{0}}\right)$.

(2) Let $p: E \rightarrow B$ be a level morphism in tow $S S_{*}\left(p=\left\{p_{i}: E_{i} \rightarrow B_{i} \mid i \in \mathbb{N}\right\}\right)$ such that each $p_{i}: E_{i} \rightarrow B_{i}$ is a fibration in $S S_{*}$. Then $\mathscr{P} p$ is a fibration in $S\left(S_{* \mathscr{P}} S^{0}\right)$.

Proof. By Definition 2.1, $\mathscr{P} p$ is a fibration in $S\left(S_{*} \mathscr{P}_{c} S^{0}\right)$ if and only if $U \mathscr{P} p=$ $\overline{\mathscr{P}}_{p}$ is a fibration in $S S_{*}$. By Lemma $1, c$ is partial left adjoint to $\overline{\mathscr{P}}:$ pro $S S_{*}$ $\rightarrow S S_{*}$. Therefore the existence of a lift in the commutative diagram

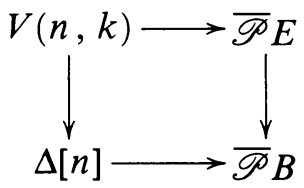


is equivalent to the existence of a lift in the corresponding commutative diagram

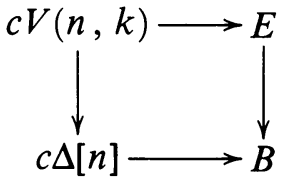

In case (1), the lift exists because pro $S S_{*}$ is a closed model category and $c V(n, k) \rightarrow c \Delta[n]$ is a trivial cofibration. For case (2), taking into account that the bonding morphisms of $c V(n, k), c \Delta[n]$ are injections, $\lim c V(n, k)=$ $\varnothing=\lim c \Delta[n], c V(n, k) \rightarrow c \Delta[n]$ is a levelwise morphism and that for each $i \geq 0 ; p_{i}: E \rightarrow B_{i}$ is a fibration, it is easy to find a lift in the diagram above.

In the following lemma, for a given closed model category $\mathscr{C}$ we use Quillen's notation $\mathscr{C}_{f}$ to denote the full subcategory of fibrant objects. We are also going to use the following notation and results: Let $S S_{*}^{\mathbb{N}}$ denote the category of functors $\mathbb{N} \rightarrow S S_{*}$ and natural transformations. Given an object $Y: \mathbb{N} \rightarrow S S_{*}$, consider $\mathbb{N}^{+}=\{-1\} \cup \mathbb{N}$ and define $Y^{+}: \mathbb{N}^{+} \rightarrow S S_{*}$ by $Y_{-1}^{+}=*$ and $Y_{i}^{+}=Y_{i}$ if $i \geq 0$. For an injective increasing map $\varphi: \mathbb{N} \rightarrow \mathbb{N}$, define $\bar{\varphi}: \mathbb{N} \rightarrow \mathbb{N}^{+}$by $\bar{\varphi}(j)=-1$ if $j \leq \varphi(0)$ and $\bar{\varphi}(j)=i$ if $\varphi(i)<j \leq \varphi(i+1)$. Now we define an object $Y^{*} \varphi: \mathbb{N} \rightarrow S S_{*}$ by $\left(Y^{*} \varphi\right)_{j}=Y_{\bar{\varphi}(j)}^{+}$. There is a natural morphism $Y \rightarrow Y^{*} \varphi$, and tow $S S_{*}$ is equivalent to the category of left fractions $\Sigma^{-1} S S_{*}^{N}$ associated with the family of morphisms of the form $Y \rightarrow Y^{*} \varphi$ (see [G-Z]). As a consequence of this fact we have that

$$
\operatorname{Hom}_{\text {tow } S S^{*}}(X, Y) \cong \operatorname{colim}_{\varphi} \operatorname{Hom}_{S S_{*}^{N}}\left(X, Y^{*} \varphi\right) .
$$

A more detailed description of these results is contained in [He.1].

We also have the functor $c: S S_{*} \rightarrow S S_{*}^{\mathbb{N}}$ defined as usual by $(c X)_{i}=\bigsqcup_{j \geq i} X$ and the functor $p: S S_{*}^{\mathbb{N}} \rightarrow S S_{*}$ defined by $p Y=\prod_{i=0}^{+\infty} Y_{i}$. It is easy to check that $c$ is left adjoint to $p$.

These results are applied to prove the second part of the following lemma that will be useful to find the relation between the proper singular functor and the right-derived functor of the $\mathscr{P}$ functor.

Lemma 3. The functor $\mathscr{P}:$ pro $S S_{*} \rightarrow S\left(S_{* \mathscr{P} c S^{0}}\right)$ satisfies the following conditions:

(1) If $f$ is a weak equivalence in ( $\left.\operatorname{pro} S S_{*}\right)_{f}$, then $\mathscr{P} f$ is a weak equivalence in $S\left(S_{* \mathscr{P} c S^{0}}\right)$. Moreover, $\mathscr{P} f$ is a homotopy equivalence in $S\left(S_{*} \mathscr{P}_{c} S^{0}\right)$.

(2) If $f=\left\{f_{i}: X_{i} \rightarrow Y_{i}\right\}$ is a level map such that for each $i \geq 0, f_{i}: X_{i} \rightarrow Y_{i}$ is a weak equivalence in $S S_{*}$ and $X_{i}, Y_{i}$ are fibrant in $S S_{*}$, then $\mathscr{P} f$ is a weak equivalence in $S\left(S_{* \mathscr{P}} S^{0}\right)$.

Proof. (1) Since $\left(\operatorname{pro} S S_{*}\right)_{f}=\left(\text { pro } S S_{*}\right)_{c f}$ and $f$ is a weak equivalence, it follows that $f$ is a homotopy equivalence. Because $\mathscr{P}: \operatorname{pro} S S_{*} \rightarrow S\left(S_{* \mathscr{D} c S^{0}}\right)$ induces a functor $\pi_{0}\left(\right.$ pro $\left.S S_{*}\right) \rightarrow \pi_{0}\left(S\left(S_{* \mathscr{D}} S^{0}\right)\right)$, we get that $\mathscr{P} f$ is also a homotopy equivalence. Therefore $\mathscr{P} f$ is a weak equivalence in $S\left(S_{* \mathscr{P}} c S^{0}\right)$.

(2) In order to prove that $\mathscr{P} f: \mathscr{P} X \rightarrow \mathscr{P} Y$ is a weak equivalence it suffices to show that $U \mathscr{P} f: U \mathscr{P} X \rightarrow U \mathscr{P} Y$ is a weak equivalence. Since for each $i \geq 0, X_{i}, Y_{i}$ are fibrant, applying (2) of Lemma 2, one has that $\mathscr{P} X, \mathscr{P} Y$ are fibrant in $S\left(S_{* \mathscr{D} c S^{0}}\right)$. Therefore $U \mathscr{P} X, U \mathscr{P} Y$ are fibrant in $S S_{*}$ and we 
obtain the following isomorphisms:

$$
\begin{aligned}
& \mid \Delta[q] / \dot{\Delta}[q], U \mathscr{P} f]=\pi_{0} \operatorname{Hom}_{S S_{*}}(\Delta[q] / \dot{\Delta}[q], U \mathscr{P} f) \\
& \quad=\pi_{0} \operatorname{Hom}_{S S_{*}}\left(\Delta[q] / \dot{\Delta}[q], \operatorname{colim}_{\varphi} p\left(f^{*} \varphi\right)\right) \\
& \quad \cong \pi_{0} \operatorname{colim}_{\varphi} \operatorname{Hom}_{S S_{*}}\left(\Delta[q] / \dot{\Delta}[q], p\left(f^{*} \varphi\right)\right) \\
& \quad \cong \pi_{0} \operatorname{colim}_{\varphi} p\left(\operatorname{Hom}_{S S_{*}}\left(\Delta[q] / \dot{\Delta}[q], f^{*} \varphi(j)\right)\right) \\
& \quad \cong \operatorname{colim}_{\varphi} p \pi_{0} \operatorname{Hom}_{S S_{*}}\left(\Delta[q] / \dot{\Delta}[q], f_{\bar{\varphi}(j)}^{+}\right) \\
& \quad \cong \operatorname{colim}_{\varphi} p\left[\Delta[q] / \dot{\Delta}[q], f_{\bar{\varphi}(j)}^{+}\right)
\end{aligned}
$$

where we have taken into account that

$$
\begin{aligned}
U \mathscr{P} X & =\operatorname{Hom}_{\text {pro } S S_{*}}\left(c \Delta[0]^{+}, X\right) \\
& \cong \operatorname{Hom}_{\text {tow } S S_{*}}\left(c \Delta[0]^{+}, X\right) \\
& \cong \operatorname{colim}_{\varphi} \operatorname{Hom}_{S S_{*}^{\mathbb{N}}}\left(c \Delta[0]^{+}, X^{*} \varphi\right) \\
& \cong \operatorname{colim}_{\varphi} \operatorname{Hom}_{S S_{*}}\left(\Delta[0]^{+}, p\left(X^{*} \varphi\right)\right) \\
& \cong \operatorname{colim}_{\varphi} p\left(X^{*} \varphi\right)
\end{aligned}
$$

and that for maps a similar expression is obtained.

Finally, since each $\left[\Delta[q] / \dot{\Delta}[q], f_{\bar{\varphi}(j)}^{+}\right]$is an isomorphism, we obtain that $[\Delta[q] / \dot{\Delta}[q], U \mathscr{P} f]$ is an isomorphism. Therefore $U \mathscr{P} f$ is a weak equivalence, and by the definition of weak equivalence in $S\left(S_{*} \mathscr{P}_{c} S^{0}\right)$ it follows that $\mathscr{P} f$ is also a weak equivalence in $S\left(S_{*} \mathscr{P}_{c} S^{0}\right)$.

Remark. Since $\mathscr{L}: S\left(S_{* \mathscr{P} c S^{0}}\right) \rightarrow$ pro $S S_{*}$ is left adjoint to $\mathscr{P}:$ pro $S S_{*} \rightarrow$ $S\left(S_{* \mathscr{P} c S^{0}}\right)$ and pro $S S_{*}, S\left(S_{* \mathscr{P} c S^{0}}\right)$ are closed model categories, it is easy to check that $\mathscr{L}$ preserves cofibrations. Since $\mathscr{L}$ is simplicial left adjoint to $\mathscr{P}$, we also get that $\mathscr{L}$ carries a weak equivalence between cofibrant objects into a weak equivalence.

Notice that Lemma 2, Lemma 3 and the remark after Lemma 3 prove that the functors $\mathscr{L}: S\left(S_{*} \mathscr{D}_{c} S^{0}\right) \rightarrow$ pro $S S_{+} *$ and $\mathscr{P}:$ pro $S S_{*} \rightarrow S\left(S_{* \mathscr{P} c S^{0}}\right)$ satisfy the conditions of Theorem 4.3 of [Q.1, Chapter I]. Therefore we have the following:

Theorem 1. The functor $\mathscr{L}: S\left(S_{* \mathscr{P} c S^{0}}\right) \rightarrow \operatorname{pro} S S_{*}$ induces a left-derived functor $\mathscr{L}^{L}: \operatorname{Ho}\left(S\left(S_{* \mathscr{P} c S^{0}}\right)\right) \rightarrow \mathrm{Ho}\left(\operatorname{pro} S S_{*}\right)$ and $\mathscr{P}: \operatorname{pro} S S_{*} \rightarrow S\left(S_{* \mathscr{D} c S^{0}}\right)$ induces a right-derived functor $\mathscr{P}^{R}: \mathrm{Ho}\left(\right.$ pro $\left.S S_{*}\right) \rightarrow \mathrm{Ho}\left(S\left(S_{* \mathscr{P}} S^{0}\right)\right)$ such that $\mathscr{L}^{L}$ is left adjoint to $\mathscr{P}^{R}$. Moreover, $\mathscr{L}^{L}$ preserves cofibration sequences and $\mathscr{P}^{R}$ preserves fibration sequences.

Recall that by Theorem 2.2 we also have the following pair of adjoint functors

$$
\operatorname{Ho}\left(S\left(S_{* \mathscr{P}_{c} S^{0}}\right)\right) \stackrel{-\odot M}{\longleftarrow} \mathrm{Ho}\left(S S_{*}\right) .
$$

The composition of the two pairs of functors gives a new pair of adjoint functors $\overline{\mathscr{L}}^{L}=\mathscr{L}^{L}(-\odot M): \mathrm{Ho}\left(S S_{*}\right) \rightarrow \mathrm{Ho}\left(\operatorname{pro} S S_{*}\right)$ and ${\mathscr{P}^{R}}^{R}=U \mathscr{P}^{R}: \mathrm{Ho}\left(\operatorname{pro} S S_{*}\right)$ $\rightarrow \mathrm{Ho}\left(S S_{*}\right)$. Therefore we have:

Corollary 1. The functor $\overline{\mathscr{L}}=\mathscr{L}(-\odot M): S S_{*} \rightarrow$ pro $S S_{*}$ has a left-derived functor $\overline{\mathscr{L}}^{L}: \mathrm{Ho}\left(S S_{*}\right) \rightarrow \mathrm{Ho}\left(\right.$ pro $\left.S S_{*}\right)$ and $\overline{\mathscr{P}}=U \mathscr{P}:$ pro $S S_{*} \rightarrow S S_{*}$ has a 
right-derived functor $\overline{\mathscr{P}}^{R}: \mathrm{Ho}\left(\operatorname{pro} S S_{*}\right) \rightarrow \mathrm{Ho}\left(S S_{*}\right)$ such that $\overline{\mathscr{L}}^{L}$ is left adjoint to $\overline{\mathscr{P}}^{R}$. Moreover $\overline{\mathscr{L}}^{L}$ preserves cofibration sequences and $\overline{\mathscr{P}}^{R}$ prescribes fibration sequences.

Notice that for a finite simplicial set $X$, we have that

$$
\mathrm{Ho}\left(\operatorname{pro} S S_{*}\right)\left(\overline{\mathscr{L}}^{L} X, Y\right) \cong \mathrm{Ho}\left(S S_{*}\right)\left(X, \overline{\mathscr{P}}^{R} Y\right) \text {. }
$$

It is easy to check that $c: S S_{*} \rightarrow$ pro $S S_{*}$ preserves cofibrations then

$$
\mathrm{Ho}\left(\operatorname{pro} S S_{*}\right)(c X, Y) \cong \mathrm{Ho}\left(\operatorname{pro} S S_{*}\right)\left(c X, Y^{\prime}\right) \text {. }
$$

where $Y \rightarrow Y^{\prime}$ is a weak equivalence and $Y^{\prime}$ is a fibrant object in pro $S S_{*}$. Since $c X$ is cofibrant and $Y^{\prime}$ is fibrant, we have

$$
\mathrm{Ho}\left(\operatorname{pro} S S_{*}\right)\left(c X, Y^{\prime}\right) \cong \pi_{0}\left(\operatorname{pro} S S_{*}\right)\left(c X, Y^{\prime}\right) .
$$

Applying Lemma 1, we see that

$$
\begin{aligned}
\pi_{0}\left(\operatorname{pro} S S_{*}\right)\left(c X, Y^{\prime}\right) & \cong \pi_{0}\left(S S_{*}\right)\left(X, \overline{\mathscr{P}} Y^{\prime}\right) \\
& \cong \pi_{0}\left(S S_{*}\right)\left(X, \overline{\mathscr{P}}^{R} Y\right) \cong \mathrm{Ho}\left(S S_{*}\right)\left(X, \overline{\mathscr{P}}^{R} Y\right) .
\end{aligned}
$$

Therefore as a consequence of these isomorphisms, we have the following:

Theorem 2. Let $\mathrm{Ho}\left(S S_{*}\right) / f$ be the full subcategory of $\mathrm{Ho}\left(S S_{*}\right)$ determined by finite simplicial sets. Then $c: \mathrm{Ho}\left(S S_{*}\right) / f \rightarrow \mathrm{Ho}\left(\operatorname{pro} S S_{*}\right)$ is a partial left adjoint to $\mathscr{\mathscr { P }}^{R}: \mathrm{Ho}\left(\operatorname{pro} S S_{*}\right) \rightarrow \mathrm{Ho}\left(S S_{*}\right)$. The functors $\overline{\mathscr{L}}^{L}$ and $c$ agree up to natural isomorphism on the subcategory $\mathrm{Ho}\left(S S_{*}\right) / f$; moreover, $c$ preserves cofibration sequences associated with a map between finite simplicial sets.

Corollary 2. The diagram

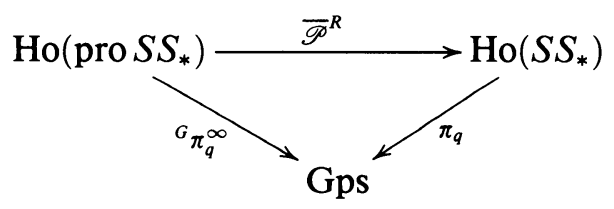

is commutative up to natural isomorphism, where $\pi_{q}$ denotes the standard qth homotopy group and ${ }^{G} \pi_{q}^{\infty}$ denotes the qth Grossman homotopy group, defined by ${ }^{G} \pi_{q}^{\infty}(X)=\mathrm{Ho}\left(\operatorname{pro} S S_{*}\right)\left(c S^{q}, X\right), S^{q}=\Delta[q] / \dot{\Delta}[q]$.

Proof. By Theorem 2 above, $c$ is partial left adjoint to $\overline{\mathscr{P}}^{R}$, so

$$
{ }^{G} \pi_{q}^{\infty}(X)=\operatorname{Ho}\left(\operatorname{pro} S S_{*}\right)\left(c S^{q}, X\right) \cong \operatorname{Ho}\left(S S_{*}\right)\left(S^{q}, \overline{\mathscr{P}}^{R} X\right) \cong \pi_{q}\left(\overline{\mathscr{P}}^{R} X\right) .
$$

Theorem 3 (Brown). The diagram

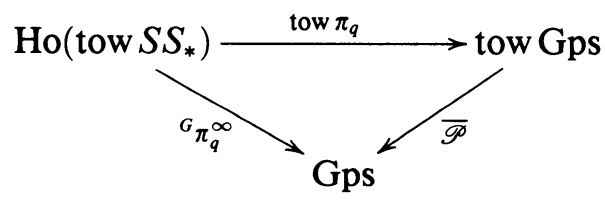


is commutative up to natural isomorphism, where tow $\pi_{q}$ is the natural prolongation of the functor $\pi_{q}$ to the category of towers.

Proof. We use again the fact that tow $S S_{*}$ can be obtained as a category of left fractions of the category $S S_{*}^{\mathbb{N}}$ (see the notation given before Lemma 3 and [He.1]). We have

$$
\begin{aligned}
{ }^{G} \pi_{q}^{\infty}(X) & =\operatorname{Ho}\left(\text { tow } S S_{*}\right)\left(c S^{q}, X\right) \\
& \cong \pi_{0} \operatorname{Hom}_{\text {tow } S S_{*}}\left(c S^{q}, R X\right) \\
& \cong \pi_{0} \operatorname{colim}_{\varphi} \operatorname{Hom}_{S S_{*}^{\mathbb{N}}}\left(c S^{q},(R X)^{*} \varphi\right) \\
& \cong \pi_{0} \operatorname{colim}_{\varphi} \operatorname{Hom}_{S S_{*}^{\mathbb{N}}}\left(S^{q}, p\left((R X)^{*} \varphi\right)\right) \\
& \cong \operatorname{colim}_{\varphi} \pi_{0} \operatorname{Hom}_{S S_{*}^{\mathbb{N}}}\left(S^{q}, p\left((R X)^{*} \varphi\right)\right) \\
& \cong \operatorname{colim}_{\varphi} \pi_{q}\left(p\left((R X)^{*} \varphi\right)\right) \\
& \cong \operatorname{colim}_{\varphi} p\left(\left(\pi_{q}(R X)\right)^{*} \varphi\right) \cong \operatorname{colim}_{\varphi} p\left(\left(\pi_{q} X\right)^{*} \varphi\right) \\
& \cong U \mathscr{P} \text { tow } \pi_{q} X \cong \overline{\mathscr{P}}_{\text {tow } \pi_{q} X}
\end{aligned}
$$

where $\pi_{q}(R X)=\left\{\pi_{q}(R X(i)) \mid i \geq 0\right\} \cong\left\{\pi_{q}(X(i)) \mid i \geq 0\right\}=\pi_{q} X$.

In Theorem 5.4, we have seen that the functors $\mathscr{P}:$ tow $S S_{*} \rightarrow S\left(S_{* \mathscr{P} c S^{0}}\right)$ and lim: tow $S S_{*} \rightarrow S S_{*}$ are related by the functor $F_{\mathrm{sh}}: S\left(S_{* \mathscr{P} c S^{0}}\right) \rightarrow S S_{*}$ in such a way that $\lim \cong F_{\text {sh }} \mathscr{P}$. The following result gives an induced relation between the right-derived functor $\lim ^{R}=$ holim of the lim functor and the right-derived functor $\mathscr{P}^{R}$ of the $\mathscr{P}$ functor. We refer the reader to [E-H] for the definition and properties of the functor holim: Ho(tow $\left.S S_{*}\right) \rightarrow S S_{*}$.

Theorem 4. The functor holim $=\lim ^{R}: \operatorname{Ho}\left(\right.$ tow $\left.S S_{*}\right) \rightarrow \operatorname{Ho}\left(S S_{*}\right)$ can be factored as

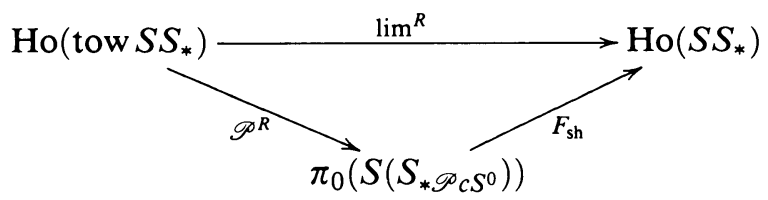

Proof. We have proved that $\mathscr{P}:\left(\text { tow } S S_{*}\right)_{f} \rightarrow S\left(S_{*} \mathscr{D}_{c} S^{0}\right)$ sends weak equivalences into simplicial homotopy equivalences. Since $F_{\text {sh }}$ preserves finite limits, it follows that $F_{\text {sh }}$ preserves homotopy relations defined by cocylinders $\left(Y^{\Delta[1]}\right)$. Therefore $F_{\mathrm{sh}}$ induces a functor $F_{\mathrm{sh}}: \pi_{0}\left(S\left(S_{*} \mathscr{P}_{c} S^{0}\right)\right) \rightarrow \mathrm{Ho}\left(S S_{*}\right)$.

Given an object $X$ in tow $S S_{*}$, we have that

$$
F_{\mathrm{sh}} \mathscr{P}^{R} X=F_{\mathrm{sh}} \mathscr{P} R X \stackrel{(1)}{\cong} \lim R X=\operatorname{holim} X,
$$

where (1) is a consequence of Theorem 5.4 and we have used the definition of holim given by Edwards and Hastings [E-H, page 133].

\section{Simplicial COMPleXes AND Simplicial $M$-SETS}

In this section we consider noncompact simplicial complexes $X$ satisfying the following three conditions.

(1) $X$ is locally finite. Each point $x \in X$ has a neighbourhood $U$ which has points in common with only a finite number of simplexes.

(2) $X$ has finite dimension. 
(3) $X$ has a countably infinite number of simplexes.

A simplicial complex of this type is homeomorphic to a subspace of some Euclidean space $\mathbb{R}^{m}$ which is the union of countably many simplexes of dimensions 0 through $n$. Two simplexes have empty intersection or they meet in a common face, and the countable family of simplexes is locally finite. A simplicial complex is said to be $n$-dimensional if it contains at least one $n$-simplex but none of higher dimension. A simplicial complex $X$ is said to be $n$-dimensional at infinity if for every finite subcomplex $K$ of $X$, there is at least an $n$-simplex of $X-K$ and none of higher dimension. In this section, simplicial complex means a simplicial complex satisfying conditions (1), (2) and (3).

Recall the functor $R_{p}:\left(S_{M} / f f\right) / f d \rightarrow$ Pro defined in $\S 4.3$, where $M=$ $\operatorname{Pro}(\mathbb{N}, \mathbb{N})$. In this section for each simplicial complex $X$, we construct a simplicial $M$-set, $N$, in $\left(S_{M} / f f\right)$ such that $R_{p} N \cong X$. The simplicial $M$-set $N$ satisfies that for each dimension $q \geq 0, N_{q}$ is $(f f)$, a free $M$-set over a finite set. The condition $f d$ means that $N$ has finite dimension, that is, there is $n$ such that for $q \geq n$ every simplex of $N_{q}$ is degenerate. The simplicial $M$-set $N$ will be proved to be cofibrant in the closed model structure of $S\left(S_{M}\right)$.

Let $X$ be a simplicial complex (satisfying (1), (2), and (3)) such that both the dimension of $X$ and the dimension of $X$ at infinity are equal to $n$. We can define a simplicial $M$-set, $N$, associated with $X$ as follows: Define $N_{0}=M$, $N_{1}=s_{0} M \sqcup M$, where $s_{0} M$ is a copy of $M$ and $\sqcup$ denotes the sum of $M$-sets. For a $k$ with $0 \leq k \leq n$, define

$$
\begin{aligned}
N_{k} & =s_{k-1} s_{k-2} \cdots s_{0} M \sqcup\left(\bigsqcup_{k>i_{k-2}>\cdots>i_{0} \geq 0} s_{i_{k-2}} \cdots s_{i_{0}} M\right) \\
& \sqcup \cdots \sqcup\left(\bigsqcup_{k>i_{1}>i_{0} \geq 0} s_{i_{1}} s_{i_{0}} M\right) \sqcup\left(\bigsqcup_{k>i_{0} \geq 0} s_{i_{0}} M\right) \sqcup M,
\end{aligned}
$$

where any $s_{i_{r}} \cdots s_{i_{0}} M$ is a copy of $M$. For $k>n, N_{k}$ is similarly defined except that the last $M$ is removed.

The degeneracy operators of $N$ are defined using the identity of $M$. Given $M$ or a copy of $M$ of the form $s_{i_{r-1}} \cdots s_{i_{0}} M$ with $k>i_{r-1}>\cdots>i_{0} \geq 0$ and $k \geq i \geq 0$, we use the relations $s_{i} s_{j}=s_{j+1} s_{i}$ if $i \leq j$ to find a copy $s_{i_{r-1}+1} \cdots s_{i} \cdots s_{i_{0}} M$ such that $i_{r-1}+1>\cdots>i>\cdots>i_{0}$. Then $s_{i}$ is defined from $s_{i_{r-1}} \cdots s_{i_{0}} M$ to $s_{i_{r-1}+1} \cdots s_{i} \cdots s_{i_{0}} M$ by the "identity" map.

To define the face operator we consider two cases: If we have a copy of $M$ of the from $s_{i_{r}} \cdots s_{i_{0}} M$ or if we have $M$. In the first case we use the relations $d_{i} s_{j}=s_{j-1} d_{i}$ if $i<j, d_{i} s_{j}=$ id if $i=j$ or $i=j+1$, and $d_{i} s_{j}=s_{j} d_{i-1}$ if $i>j+1$ to transform an expression of the form $d_{i} s_{i_{r}} \cdots s_{i_{0}} M$ into an expression of the form $s_{j_{r-1}} \cdots s_{j_{0}} M$. Then the restriction of the face operator $d_{i}$ to $s_{i_{r}} \cdots s_{i_{0}} M$ is defined by the "identity" map from $s_{i_{r}} \cdots s_{i_{0}} M$ to $s_{j_{r-1}} \cdots s_{j_{0}} M$.

Now we have to define the face operators for the term of $N_{k}(1 \leq k \leq n)$ equal to $M$. It is in this step where we use the combinatorial structure of the simplicial complex $X$.

Given a simplicial complex $X$ (satisfying (1), (2), and (3)) such that the dimension of $X$ and the dimension of $X$ at infinity are equal to $n$, firstly, an enumeration can be chosen for the countable set of 0-simplexes of $X, E_{0}^{0}, E_{1}^{0}$, 
$E_{2}^{0}$, etc. This enumeration induces a unique order to the finite set of vertices of each $k$-simplex $E^{k}$ of $X$. Therefore for each $k$-simplex $E^{k}$ of $X$ the different faces $d_{0} E^{k}, d_{1} E^{k}, \ldots, d_{k} E^{k}$ are well defined. We also choose an enumeration for the countable set of 1-simplexes of $X$, the countable set of 2 -simplexes, etc, and finally for the countable set of $n$-simplexes.

If $0<k \leq n, 0 \leq i \leq k$, for each $l \in \mathbb{N}$ the face $d_{i} E_{l}^{k}$ is equal to some $E_{\varphi_{i} l}^{k-1}$. This defines a proper map $\varphi_{i}: \mathbb{N} \rightarrow \mathbb{N}$; that is, an element $\varphi_{i} \in M$. The restriction of the face operator $d_{i}$ applies the term $M$ of $N_{k}$ into the term $M$ of $N_{k-1}$. Since $M$ is a right $M$-set freely generated by $1 \in M$, it suffices to define $d_{0} 1=\varphi_{0}, \ldots, d_{k} 1=\varphi_{k}$.

The simplicial $M$-set $N$ satisfies that $R_{p} N \cong X$, where $R_{p}:\left(S_{M} / f f\right) / f d \rightarrow$ Pro is the realization functor defined in $\S 4.3$. The reason for this is that the space $X$ admits the following inductive construction. We start with a "proper" 0 -simplex $\mathbb{N} \times|\Delta[0]|$. We attach a "proper" 1-simplex to obtain the 1-skeleton, and continue in this way to obtain the $n$-skeleton of $X$. On the other hand, if we look at the definition of $R_{p} N$ and take into account Proposition 3.2, we have to consider the diagram $D\left(S k_{n} N\right)$ (see $\S 3$ ). In this case, because $s_{j} 1_{M}=1_{M}$ we can again reduce $D\left(S k_{n} N\right)$ to a diagram that contains exactly the necessary instructions to attach each face $\mathbb{N} \times\left|\partial_{i} \Delta[q]\right|$ of the "proper" $q$ simplex $\mathbb{N} \times|\Delta[q]|$.

Notice that the realization functor satisfies

$$
\begin{aligned}
& R_{p}(\Delta[q] \odot M) \cong \mathbb{N} \times|\Delta[q]| \cong \bigsqcup_{\mathbb{N}}|\Delta[q]|, \\
& R_{p}(\dot{\Delta}[q] \odot M) \cong \mathbb{N} \times|\dot{\Delta}[q]| \cong \bigsqcup_{\mathbb{N}}|\dot{\Delta}[q]| .
\end{aligned}
$$

For the case when both the dimension of $X$ and the dimension of $X$ at infinity are equal to $n$, we have constructed a simplicial $M$-set, $N$, such that $R_{p} N \cong X$. For the general case we have $\operatorname{dim} X=m \geq n$, where $n$ is the dimension of $X$ at infinity. We note that there are finitely many simplexes of dimension greater than $n$. Using the construction above we can find a simplicial $M$-set, $N^{\prime}$, such that $R_{p} N^{\prime} \cong \mathrm{sk}_{n} X$. In order to attach the simplexes of dimension greater than $n$, for each $q \geq n$, we are going to construct a simplicial $M$-set $\Delta_{1}[q]$ such that $R_{p} \Delta_{1}[q] \cong|\Delta[q]| \sqcup\left(\bigsqcup_{1}^{+\infty} *\right)$. Now instead of attaching $|\Delta[q]|$ by using a map $|\dot{\Delta}[q]| \rightarrow \operatorname{sk}_{q-1} X$, we attach $|\Delta[q]| \sqcup\left(\bigsqcup_{1}^{+\infty} *\right)$ by using a proper map $|\dot{\Delta}[q]| \sqcup\left(\bigsqcup_{1}^{+\infty} *\right) \rightarrow \mathbf{s k}_{q-1} X$.

We note that if $Y$ is a simplicial $M$-set, we have the following isomorphisms:

$$
\operatorname{Hom}_{S\left(S_{M}\right)}(\Delta[q] \odot M, Y) \cong \operatorname{Hom}_{S S}(\Delta[q], U Y) \cong U Y_{q},
$$

where $U$ is right adjoint to $-\odot M$. Therefore each element $y \in Y_{q}$ determines a map $f_{y}: \Delta[q] \odot M \rightarrow Y$. Recall that for each simplicial set $Z$, we have that $(Z \odot M)_{q} \cong Z_{q} \odot M \cong Z_{q} \times M$, and an element $(z, m)$ of $Z_{q} \odot M$ is also denoted by $z \odot m$. If $i_{q}$ denotes the identity of $[q]$ and sh: $\mathbb{N} \rightarrow \mathbb{N}$ is an element of $M$ defined by $\operatorname{sh}(i)=i+1$, we have that the element $i_{q} \odot$ sh of $(\Delta[q] \odot M)_{q}$ determines a map $f_{i_{q} \odot \text { sh }}: \Delta[q] \odot M \rightarrow \Delta[q] \odot M$. We also consider the restriction of $f_{i_{q} \odot \text { sh }}$ to the corresponding $(q-1)$-skeletons that will be denoted by $\operatorname{sk}_{q-1}\left(f_{i_{q} \odot \text { sh }}\right)$. On the other hand the final map $*: \Delta[q] \rightarrow \Delta[0]$ induces a map $* \odot M: \Delta[q] \odot M \rightarrow \Delta[0] \odot M$. Using this notation, the simplicial 
$M$-set $\Delta_{1}[q]$ is determined by the pushout

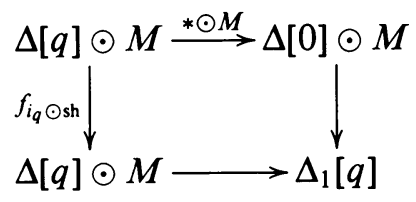

and similarly one also has the pushout

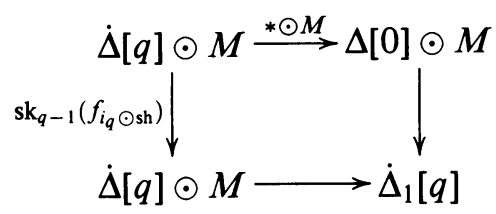

It is easy to check that

$$
\begin{aligned}
& R_{p} \Delta_{1}[q] \cong|\Delta[q]| \sqcup\left(\bigsqcup_{1}^{+\infty} *\right), \\
& R_{p} \dot{\Delta}_{1}[q] \cong|\dot{\Delta}[q]| \sqcup\left(\bigsqcup_{1}^{+\infty} *\right) .
\end{aligned}
$$

If we suppose that we have a simplicial $M$-set $N^{\prime}$ such that $R_{p} N^{\prime} \cong \mathrm{sk}_{n} X$. Because there are finitely many simplexes with dimension greater than $n$, we can consider pushouts of the form $N^{\prime \prime}=N^{\prime} \sqcup_{\dot{\Delta}_{1}[p]} \Delta_{1}[p]$ to obtain finally the desired $N$.

Notice that the simplicial $M$-set $N$ has the following skeletal structure:

$$
\mathbf{s k}_{0} N \subset \mathbf{s k}_{1} N \subset \cdots \subset \mathbf{s k}_{n} N \subset \cdots \subset \mathbf{s k}_{m} N,
$$

where if $l \leq n, \mathrm{sk}_{l} N$ is obtained from $\mathrm{sk}_{l-1} N$ by a pushout of the form

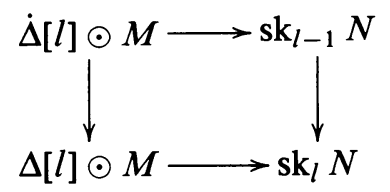

where the map $\Delta[l] \odot M \rightarrow \mathbf{s k}_{l} N$ is determined by the adjoint isomorphisms by the identity 1 of $M$ considered as an element of the term $M$ of $\left(\mathbf{s k}_{l} N\right)_{l} \cong N_{l}$. If $l>n, N_{l}$ is obtained from $N_{l-1}$ by a pushout of the form

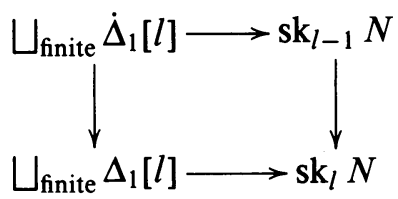

Since $\dot{\Delta}_{1}[l] \rightarrow \Delta_{1}[l]$ is a retract of $\dot{\Delta}[l] \odot M \rightarrow \Delta[l] \odot M$, which is a cofibration, it follows that $\dot{\Delta}_{1}[l] \rightarrow \Delta_{1}[l]$ and $\dot{\Delta}[l] \odot M \rightarrow \Delta[l] \odot M$ are cofibrations in $S\left(S_{M}\right)$. Therefore $N$ is a cofibrant object in $S\left(S_{M}\right)$. It is also clear that $N$ is an object of $\left(S_{M} / f f\right) / f d$.

Then we have proved the following: 
Theorem 1. For any simplicial complex $X$, there is an object $N$ in $\left(S_{M} / f f\right) / f d$ which is cofibrant in $S\left(S_{M}\right)$ and such that $R_{p} N \cong X$.

We are going to analyse the relationship between the proper realization functor $R_{p}: S\left(S_{M} / f f\right) / f d \rightarrow$ Pro and the realization functor $\mathscr{L}=R_{\chi_{\infty}^{g}}: S\left(S_{M}\right) \rightarrow$ (pro $S S, S S$ ). Consider the Edwards-Hastings embedding

$$
\varepsilon: \text { Pro } \rightarrow \text { (pro Top, Top) }
$$

and the restrictions $\varepsilon:$ Pro $_{\sigma} \rightarrow$ (pro Top, Top) and $\varepsilon: P C \rightarrow$ (pro Top, Top), where $\mathrm{Pro}_{\sigma}$ is the full subcategory of Pro determined by locally compact, $\sigma$ compact Hausdorff spaces and $P C$ is the full subcategory of Pro $_{\sigma}$ determined by spaces that admit a triangulation as a simplicial complex satisfying the conditions (1), (2) and (3) at the beginning of the section.

Edwards and Hastings [E-H; Proposition 6.2.7] proved that the induced functors

$$
\begin{gathered}
\varepsilon: \pi_{0}\left(\text { Pro }_{\sigma}\right) \rightarrow \text { Ho }_{\mathrm{St}}(\text { pro Top }, \text { Top }), \\
\varepsilon: \pi_{0}\left(\left(\text { Pro }_{\sigma}\right)_{\infty}\right) \rightarrow \text { Ho }_{\mathrm{St}}(\text { pro Top })
\end{gathered}
$$

are full embeddings, where $\pi_{0}\left(\operatorname{Pro}_{\sigma}\right)$ and $\pi_{0}\left(\left(\operatorname{Pro}_{\sigma}\right)_{\infty}\right)$ are defined dividing by proper homotopies and germs of proper homotopies and $\mathrm{Ho}_{\mathrm{st}}$ (pro Top, Top), $\mathrm{Ho}_{\mathrm{st}}$ (pro Top) are obtained by the inversion of the weak equivalences of (pro Top, Top) (resp., pro Top) of the closed model structure defined by Edwards and Hastings [E-H] on these procategories and induced by the Strøm closed model structure of Top.

If one considers the closed simplicial model structure of Top defined by Quillen [Q.1], using the Edwards-Hastings method there are induced closed simplicial model structures on the categories (pro Top, Top) and pro Top. Let $\mathrm{Ho}_{Q}$ (pro Top, Top), $\mathrm{Ho}_{Q}$ (pro Top) denote the corresponding localized categories. Using these new closed model structures, there are also full embeddings

$$
\begin{gathered}
\varepsilon: \pi_{0}(P C) \rightarrow \mathrm{Ho}_{Q} \text { (pro Top, Top), } \\
\varepsilon:\left(\pi_{0}(P C)_{\infty}\right) \rightarrow \mathrm{Ho}_{Q} \text { (pro Top), }
\end{gathered}
$$

if we consider the restriction of $\varepsilon$ to spaces that admit a triangulation as a simplicial complex.

The standard realization and singular functor

$$
\text { Top } \underset{S}{\stackrel{R}{\leftrightarrows}} S S
$$

induce equivalences of categories

$$
\begin{gathered}
\mathrm{Ho}_{Q}(\text { pro Top, Top }) \underset{S}{\stackrel{R}{\leftrightarrows}} \mathrm{Ho}(\text { pro } S S, S S), \\
\mathrm{Ho}_{Q}(\text { pro Top }) \underset{S}{\stackrel{R}{\leftrightarrows}} \mathrm{Ho}(\text { pro } S S) .
\end{gathered}
$$

Therefore we also have the full embeddings

$$
\begin{gathered}
S \varepsilon: \pi_{0}(P C) \rightarrow \mathrm{Ho}(\operatorname{pro} S S, S S), \\
S \varepsilon: \pi_{0}\left((P C)_{\infty}\right) \rightarrow \mathrm{Ho}(\operatorname{pro} S S) .
\end{gathered}
$$

The next proposition relates the proper realization functor $R_{p}:\left(S_{M} / f f\right) / f d$ $\rightarrow P C$ and the realization functor $\mathscr{L}: S\left(S_{M}\right) \rightarrow($ pro $S S, S S)$. 
Proposition 1. Let $X$ be an object of $P C$ and let $N$ be a simplicial $M$-set associated with $X$ by the construction given in this section $\left(R_{p} N \cong X\right)$. Then $\mathscr{L} N$ is isomorphic to $S \varepsilon X$ in the category $\mathrm{Ho}(\operatorname{pro} S S, S S)$.

Proof. Let $X$ be a simplicial complex and assume that the set of vertexes of each simplex of $X$ is provided with a fixed order. We can define a simplicial set $s X$ by

$$
(s X)_{q}=\{f:|\Delta[q]| \rightarrow X \mid f \text { is a simplicial, order-preserving map }\} .
$$

It is well known that $s X \rightarrow S X$ is a weak equivalence in $S S$. Therefore if $X$ is an object of $P C$ provided with an enumeration for the countable set of its vertexes and $X=X(0) \supset X(1) \supset \cdots$ is a decreasing sequence of subcomplexes such that $X(i+1) \subset$ Int $X(i), i \geq 0$, and $\bigcap X(i)=\varnothing$, we have that $s \varepsilon^{\prime} X \rightarrow$ $S \varepsilon X$ is a weak equivalence in (pro $S S, S S$ ), where $s \varepsilon^{\prime} X=\{s X(i)\}$.

Assume that $X$ is an object in $P C$ with $\operatorname{dim} X=m$ and the dimension of $X$ at infinity is equal to $n(m \geq n)$. Suppose that $X$ is provided with the corresponding enumerations for the countable sets of 0 -simplexes, 1 -simplexes, ... and $n$-simplexes. Then for $1 \leq l \leq n$ we have the following pushouts:

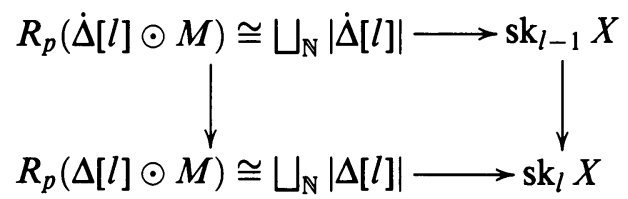

and for $n<l \leq m$

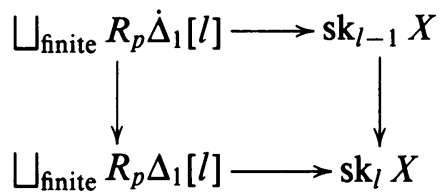

The "functor" $s \varepsilon^{\prime}$ preserves these colimits and we have, in (pro $S S, S S$ ), the pushouts

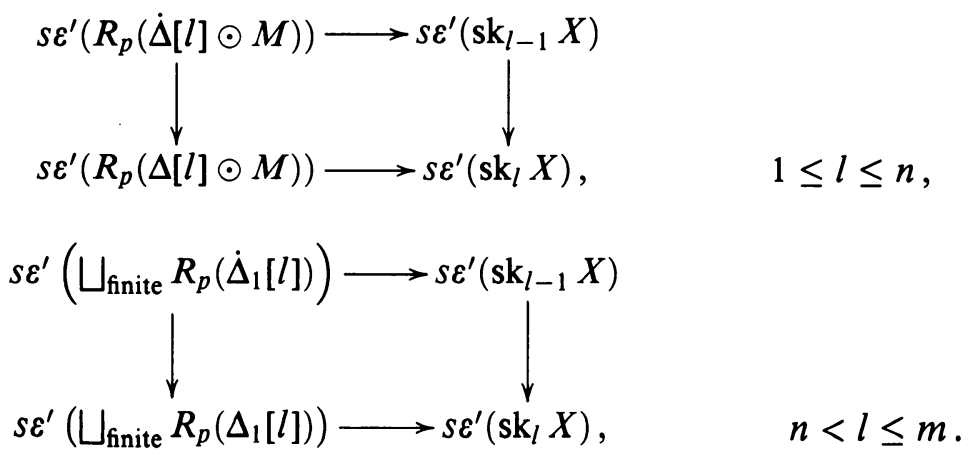

The left adjoint $\mathscr{L}: S\left(S_{M}\right) \rightarrow($ pro $S S, S S)$ preserves colimits, so for the simplicial $M$-set $N$ we have the sequence

$$
\mathscr{L} \mathbf{s k}_{0} N \subset \mathscr{L} \mathbf{s k}_{1} N \subset \cdots \subset \mathscr{L} \mathbf{s k}_{n} N \subset \cdots \subset \mathscr{L} \mathbf{s k}_{m} \cong \mathscr{L} N
$$


and the pushouts

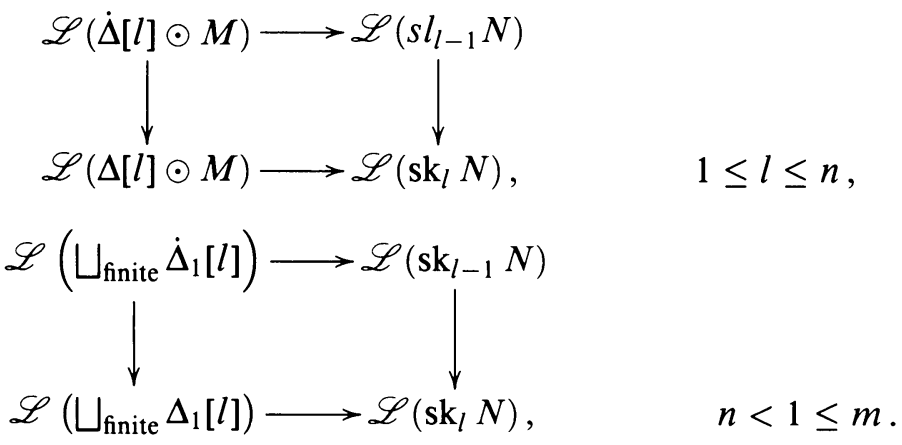

But we have

$$
\begin{array}{cl}
\mathscr{L}(\dot{\Delta}[l] \odot M) \cong s \varepsilon^{\prime}\left(R_{p}(\dot{\Delta}[l] \odot M)\right), & \mathscr{L}(\Delta[l] \odot M) \cong S \varepsilon^{\prime}\left(R_{p}(\Delta[l] \odot M)\right), \\
\mathscr{L}\left(\dot{\Delta}_{1}[l]\right) \cong s \varepsilon^{\prime}\left(R_{p} \dot{\Delta}_{1}[l]\right), & \mathscr{L}\left(\Delta_{1}[l]\right) \cong s \varepsilon^{\prime}\left(R_{p} \Delta_{1}[l]\right) .
\end{array}
$$

Then by induction it follows that $\mathscr{L}\left(\mathrm{sk}_{0} N\right) \cong s \varepsilon^{\prime} \mathrm{sk}_{0} X, \mathscr{L}\left(\mathrm{sk}_{1} N\right) \cong s \varepsilon^{\prime} \mathrm{sk}_{1} X$, $\ldots$, and finally $L N \cong s \varepsilon^{\prime} X$. Therefore $S \varepsilon X$ is isomorphic in $\operatorname{Ho}(\operatorname{pro} S S, S S)$ to $\mathscr{L}(N)$, where $N$ is an object of $\left(S_{M} / f f\right) / f d$ which is cofibrant in $S\left(S_{M}\right)$.

\section{APPLICATIONS TO PROPER HOMOTOPY THEORY}

Associated with the monoid $M=\operatorname{Pro}(\mathbb{N}, \mathbb{N})$, we have introduced the proper realization functor $R_{p}:\left(S_{M} / f f\right) / f d \rightarrow$ Pro and the proper singular functor $S_{p}$ : Pro $\rightarrow S\left(S_{M}\right)$. Given an object $N$ of $S\left(S_{M} / f f\right) / f d$ and a space $Y$, by Theorem 4.1 we have that $\pi_{0}$ (Pro) $\left(R_{p} N, Y\right) \cong \pi_{0}\left(S\left(S_{M}\right)\right)\left(N, S_{p} Y\right)$. If $N$ is also a cofibrant object in $S\left(S_{M}\right)$, then Theorem 4.2 implies that $\pi_{0}($ Pro $)\left(R_{p} N, Y\right) \cong \operatorname{Ho}\left(S\left(S_{M}\right)\right)\left(N, S_{p} Y\right)$. Consequently, in some cases, the problem of computing sets of proper homotopy classes is translated from the proper homotopy category $\pi_{0}$ (Pro) to the category of fractions $\operatorname{Ho}\left(S\left(S_{M}\right)\right)$.

We note that the definition of the functor $S_{p}$ is given by sequences of singular simplexes converging to infinity. Therefore the use of the functors $R_{p}$ and $S_{p}$ will be more convenient for spaces which are first countable at infinity. For more general spaces we have to use nets instead of sequences, and the category $S\left(S_{M}\right)$ would have to be modified to one of the form $S\left(S_{\mathscr{M}}\right)$ where $\mathscr{M}$ is a category of "proper maps" between directed sets. In any case, many of the more important applications of the proper homotopy theory are concerned with noncompact spaces which are first countable at infinity.

An important class of these latter spaces are the simplicial complexes considered in $\S 7$. Recall that $P C$ denotes the category of proper maps between spaces that admit a simplicial decomposition with a countably infinite number of simplexes; we also assume that this triangulation is locally finite and has finite dimension. By Theorem 7.1, a simplicial complex $X$ of $P C$ is of form $X \cong R_{p} N$, where $N$ is an object of $S\left(S_{M} / f f\right) / f d$ which is cofibrant in $S\left(S_{M}\right)$. Then it follows that

$$
\pi_{0}(\operatorname{Pro})(X, Y) \cong \pi_{0}(\operatorname{Pro})\left(R_{p} N, Y\right) \cong \operatorname{Ho}\left(S\left(S_{M}\right)\right)\left(N, S_{p} Y\right) \text {. }
$$

In order to define the proper homotopy groups of a space $X$, we choose a base sequence $\sigma: \mathbb{N} \rightarrow X$ converging to infinity. Associated with $X$, one has 
the simplicial $M$-set $S_{p} X$, and the forgetful functor $U: S\left(S_{M}\right) \rightarrow S S$ gives the simplicial set $\bar{S}_{p} X=U S_{p} X$. Notice that $\sigma$ is a 0 -simplex of $\bar{S}_{p} X$. We consider the following definition of proper homotopy groups ${ }^{p} \pi_{q}(X, \sigma)$.

Definition 1. Let $X$ be a space and $\sigma: \mathbb{N} \rightarrow X$ a proper map. Then the $q$ th proper homotopy group is defined by

$$
{ }^{p} \pi_{q}(X, \sigma):=\pi_{q}\left(\bar{S}_{p} X, \sigma\right) .
$$

Remarks. (1) For the category Pro $_{\infty}$ of germs of proper maps and the monoid $M_{\infty}=\operatorname{Pro}_{\infty}(\mathbb{N}, \mathbb{N})$, we have similar notions and results. For instance, we can consider the proper homotopy groups at infinity ${ }^{p} \pi_{q}^{\infty}(X, \sigma)$ of a space $X$ and base sequence $\sigma$.

(2) E. M. Brown [Br.1] define the proper homotopy groups ${ }^{B} \pi_{q}^{\infty}(X, \alpha)$ of a space $X$ with a proper base ray $\alpha:[0,+\infty) \rightarrow X$. If $S^{q}$ denotes the $q$ sphere and $*$ is a base point of $S^{q}$, we can consider the Brown $q$-sphere ${ }^{B} S^{q}=([0, \infty) \times\{*\}) \cup\left(\mathbb{N} \times S^{q}\right)$. It is easy to check that the inclusion $\mathbb{N} \times S^{q} \rightarrow$ $([0, \infty) \times\{*\}) \cup\left(\mathbb{N} \times S^{q}\right)$ induces a group isomorphism $\eta_{\alpha}:{ }^{B} \pi_{q}^{\infty}(X, \alpha) \rightarrow$ ${ }^{p} \pi_{q}^{\infty}(X, \alpha / \mathbb{N})$. We note that if $\alpha, \alpha^{\prime}:[0, \infty) \rightarrow X$ are two proper rays such that $\alpha / \mathbb{N}=\alpha^{\prime} / \mathbb{N}$, we have the group isomorphism $\theta=\eta_{\alpha^{\prime}}^{-1} \eta_{\alpha}:{ }^{B} \pi_{q}^{\infty}(X, \alpha) \rightarrow$ ${ }^{B} \pi_{q}^{\infty}\left(X, \alpha^{\prime}\right)$. However, two different choices of base ray can lead to nonisomorphic progroups. We refer the reader to Siebenmann's thesis [Sie.1]. He considers a space $X$ (an infinite cylinder with an infinite string of circles) and two proper maps $\alpha, \alpha ;:[0, \infty) \rightarrow X$ that lead to nonisomorphic pro-groups

$$
G=\text { tow } \pi_{1}(\varepsilon(X, \alpha)) \not \text { tow } \pi_{1}\left(\varepsilon\left(X, \alpha^{\prime}\right)\right)=G^{\prime} .
$$

Siebenmann shows that for $\alpha, \lim G$ is a cyclic infinite group, and for $\alpha^{\prime}$, $\lim G^{\prime}$ is a trivial group. Recall that if we consider the functor $\mathscr{P}:$ tow Grp $\rightarrow$ $\operatorname{Grp}_{\mathscr{P} c \mathbb{Z}}$, for the progroups $G, G^{\prime}$ we have the group isomorphisms:

$$
\begin{aligned}
\lim G \cong\{x \in \mathscr{P} G \mid x \mathrm{sh}=x\}, \\
\lim G^{\prime} \cong\left\{x^{\prime} \in \mathscr{P} G^{\prime} \mid x^{\prime} \mathrm{sh}=x^{\prime}\right\} .
\end{aligned}
$$

Therefore, as a consequence of Siebenman's example we obtain that $\mathscr{P} G$ is not isomorphic to $\mathscr{P} G^{\prime}$ in the category $\mathrm{Grp}_{\mathscr{P} c \mathbb{Z}}$ (notice that any morphism in $\mathrm{Grp}_{\mathscr{P}_{c \mathbb{Z}}}$ has to "commute" with the shift operator sh).

On the other hand, by Theorem 6.3, one has canonical isomorphisms

$$
\overline{\mathscr{P}}_{G} \cong{ }^{B} \pi_{1}^{\infty}(X, \alpha), \quad \overline{\mathscr{P}} G^{\prime} \cong{ }^{B} \pi_{1}^{\infty}\left(X, \alpha^{\prime}\right) .
$$

In the Siebenmann example we have that the group isomorphism $\theta$ does not preserve the action of sh, hence $\theta$ is not a morphism of the category $\operatorname{Grp}_{\mathscr{P} c \mathbb{Z}}$.

Now we obtain the following version of the Whitehead theorem in the proper setting.

Theorem 1. Let $f: X \rightarrow Y$ be a proper map between simplicial complexes (that is, $f$ is a morphism of $P C$ ). Then $f$ is a proper homotopy equivalence if and only if ${ }^{p} \pi_{q}(f):{ }^{p} \pi_{q}(X, \sigma) \rightarrow{ }^{p} \pi_{q}(Y, f \sigma)$ is an isomorphism for all $q \geq 0$ and for every base sequence $\sigma$.

Proof. Let $Z$ be a object in $P C$. By Theorem 7.1, there is an object $N$ in $S\left(S_{M} / f f\right) / f d$ which is cofibrant in $S\left(S_{M}\right)$ and such that $Z \cong R_{p} N$. Using 
Theorem 7.2, we obtain the following commutative diagram:

$$
\begin{aligned}
\pi_{0}(\operatorname{Pro})\left(R_{p} N, X\right) \cong \operatorname{Ho}\left(S\left(S_{M}\right)\right)\left(N, S_{p} X\right) \\
\pi_{*}\left(\operatorname{Pro}\left(R_{p} N, Y\right)\right) \cong \operatorname{Ho}\left(S\left(S_{M}\right)\right)\left(N, S_{p} Y\right)
\end{aligned}
$$

By the definition of ${ }^{p} \pi_{q}$, one has that $\bar{S}_{p} f=U S_{p} f$ is a weak equivalence in $S S$. Taking into account the definition of weak equivalence in $S\left(S_{M}\right)$, we have that $S_{p} f$ is a weak equivalence. Therefore $\left(S_{p} f\right)_{*}$ is an isomorphism in the diagram above, and this implies that $f_{*}$ is also an isomorphism. This follows for any $Z \cong R_{p} N$, and by the Yoneda lemma one obtains that $f$ is an isomorphism in $\pi_{0}$ (Pro); that is, $f$ is a proper homotopy equivalence.

Remarks. (1) A similar version of this paper Whitehead theorem can be proved for germs of proper maps and the proper homotopy groups at infinity ${ }^{p} \pi_{q}^{\infty}$.

(2) Siebenmann [Sie.2], Farrel-Taylor-Wagoner [F-T-W], Edwards-Hastings [E-H] and Bassendoski [Bas] have proved different versions of the proper Whitehead theorem. There are also other versions of the Whitehead theorem for prospaces and prosimplicial sets that can be applied to proper homotopy. Extremiana-Hernández-Rivas [E-H-R.1] gave a version that only uses strong (Steenrod) proper homotopy groups. Baues [Ba.2] and Ayala-DomínguezQuintero have given a Whitehead theorem for spaces with a base tree.

(3) Let $\pi={ }^{p} \pi_{1}(X)$ and assume that there is an action of $\pi$ on an abelian group $A$. One can define proper cohomology of $X$ with twisted coefficients by ${ }^{p} H^{q}(X ; A):=H^{q}\left(\bar{S}_{p} X ; A\right)$. It is clear that the cohomology version of the standard Whitehead theorem implies a similar version for the proper category.

The following result gives the relation between the proper singular functor, the right-derived functor of the $\mathscr{P}$ functor, and the Edwards-Hasting functor.

Theorem 2. Let Pro $_{\sigma}$ be the full subcategory of Pro determined by locally compact, $\sigma$-compact Hausdorff spaces. Then the diagram

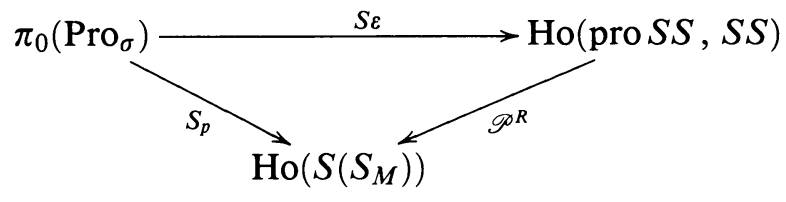

is commutative up to natural isomorphism.

Proof. Let $X$ be an object in Pro $_{\sigma}$. From the topological properties of $X$, we infer that $\varepsilon X \cong\left\{X_{i} \mid i \in \mathbb{N}\right\}$. Therefore $S \varepsilon X \cong\left\{S X_{i}\right\}$. Using the properties of the model structure of (tow $S S, S S$ ), one has a levelwise map $\left\{f_{i}: S X_{i} \rightarrow\right.$ $\left.(R S \varepsilon X)_{i}\right\}$ such that $R S \varepsilon X$ is a fibrant object, and for each $i \geq 0, S X_{i}$ and $(R S \varepsilon X)_{i}$ are fibrant objects, and $f_{i}$ is a weak equivalence. We can now apply Lemma 6.3, to obtain that

$$
\mathscr{P} S \varepsilon X \cong \mathscr{P}\left\{S \varepsilon X_{i}\right\} \rightarrow \mathscr{P}(R S \varepsilon X)=\mathscr{P}^{R} S \varepsilon X
$$

is a weak equivalence; that is, an isomorphism in $\mathrm{Ho}\left(S\left(S_{M}\right)\right)$. 
On the other hand, one has the isomorphisms

$$
\begin{aligned}
(\mathscr{P} S \varepsilon X)_{q} & \cong(\operatorname{pro} S S, S S)(\mathscr{L}(\Delta[q] \odot M), S \varepsilon X) \\
& \cong(\operatorname{proTop}, \text { Top })(|c \Delta[q]|, \varepsilon X) \\
& \cong(\operatorname{proTop}, \text { Top })(\varepsilon(\mathbb{N} \times|\Delta[q]|), \varepsilon X) \\
& \cong \operatorname{Pro}(\mathbb{N} \times|\Delta[q]|, X) \cong\left(S_{p} X\right)_{q} .
\end{aligned}
$$

Therefore $\mathscr{P} S \varepsilon X$ is isomorphic to $S_{p} X$ and $S_{p} X \rightarrow \mathscr{P}^{R} S \varepsilon X$ is an isomorphism in $\mathrm{Ho}\left(S\left(S_{M}\right)\right)$.

A partial version of the Edwards-Hastings embedding theorem can be obtained as a corollary.

Corollary 1. Let $X$ be an object in $P C$. Then

$$
\pi_{0}(\operatorname{Pro})(X, Y) \cong \mathrm{Ho}(\operatorname{pro} S S, S S)(S \varepsilon X, S \varepsilon Y)
$$

for any space $Y$ in $\mathrm{Pro}_{\sigma}$.

Proof. By Theorem 7.1 there is an object $N$ in $S\left(S_{M}\right)$ such that $R_{p} N \cong X$ and

$$
\pi_{0}(\operatorname{Pro})(X, Y) \cong \mathrm{Ho}\left(S\left(S_{M}\right)\right)\left(N, S_{p} Y\right) .
$$

By the above theorem, $S_{p} Y$ is isomorphic to $\mathscr{P}^{R} S \varepsilon Y$ in $\operatorname{Ho}\left(S\left(S_{M}\right)\right)$, so one has

$$
\begin{aligned}
\operatorname{Ho}\left(S\left(S_{M}\right)\right)\left(N, S_{p} Y\right) & \cong \mathrm{Ho}\left(S\left(S_{M}\right)\right)\left(N, \mathscr{P}^{R} S \varepsilon Y\right) \\
& \cong \mathrm{Ho}(\operatorname{pro} S S, S S)(\mathscr{L} N, S \varepsilon Y) .
\end{aligned}
$$

In the last isomorphism, we have taken into account that $N$ is cofibrant in $S\left(S_{M}\right)$. Applying Proposition 7.1, one has that $\mathscr{L} N$ is isomorphic to $S \varepsilon X$. Therefore

$$
\pi_{0}(\text { Pro })(X, Y) \cong \mathrm{Ho}(\operatorname{pro} S S, S S)(S \varepsilon X, S \varepsilon Y) .
$$

Different homology theories can be defined in order to have Hurewicz theorems. If one considers the following definition, we have that the standard Hurewicz theorem implies a proper Hurewicz theorem. Recall that $\bar{S}_{p} X$ denotes the simplicial set $U S_{p} X$, where $U: S\left(S_{M}\right) \rightarrow S S$ is the forgetful functor and $S_{p}$ is the proper singular functor.

Definition 2. Let $X$ be a space. The $q$ th proper homology group of $X$ is defined by ${ }^{p} H_{q}(X):=H_{q}\left(\bar{S}_{p} X\right)$.

Theorem 3 (Proper Hurewicz theorem). Let $X$ be a noncompact space and suppose that $X$ is properly 0 -connected $\left({ }^{p} \pi_{0}(X, \sigma)=*\right.$ for some base sequence $\sigma)$. Then there is a homomorphism ${ }^{p} \pi_{q}(X) \rightarrow{ }^{p} H_{q}(X)$ for each $q \geq 0$ such that:

(1) For $q=1,{ }^{p} \pi_{1}(X) \rightarrow{ }^{p} H_{1}(X)$ is up to isomorphism the natural epimorphism from a group to its abelianization. The first proper homology group is isomorphic to the abelianization of the proper fundamental group.

(2) If $X$ is properly $(n-1)$-connected, $n \geq 2\left({ }^{p} \pi_{q}(X) \cong 0\right.$ for $\left.q \leq n-1\right)$, then the Hurewicz homomorphism ${ }^{p} \pi_{n}(X) \rightarrow{ }^{p} H_{n}(X)$ is an isomorphism and ${ }^{p} \pi_{n+1}(X) \rightarrow{ }^{p} H_{n+1}(X)$ is an epimorphism. 
Remarks. (1) If $X$ is a space and $\alpha:[0,+\infty) \rightarrow X$ a base ray, such that $\alpha$ is a "proper cofibration", then the pushout

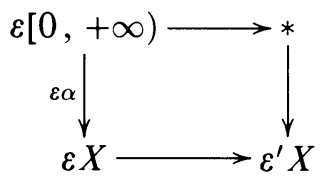

defines an object $\varepsilon^{\prime} X$ of (pro Top ${ }_{*}$, Top ${ }_{*}$ ) and $\varepsilon X \rightarrow \varepsilon^{\prime} X$ is a weak equivalence in (pro Top, Top). Then $\overline{\mathscr{P}}^{R} S \varepsilon X \rightarrow \overline{\mathscr{P}} S \varepsilon^{\prime} X$ is a weak equivalence in $S S$, and we have that $\pi_{q}\left(\overline{\mathscr{P}}^{R} S \varepsilon X\right) \cong \pi_{q}\left(\overline{\mathscr{P}}^{R} S \varepsilon^{\prime} X\right)$. The proper homotopy groups satisfy

$$
{ }^{p} \pi_{q}(X)=\pi_{q}\left(S_{p} X\right) \cong \pi_{q}\left(\overline{\mathscr{P}}^{R} S \varepsilon X\right) \cong \pi_{q}\left(\overline{\mathscr{P}}^{R} S \varepsilon^{\prime} X\right) \cong \overline{\mathscr{P}}\left(\text { pro } \pi_{q}, \pi_{q}\right)\left(\varepsilon^{\prime} X\right) .
$$

That is, the functor $\pi_{q}$ commutes with the $\overline{\mathscr{P}}$ functor.

(2) The functor $H_{q}$ does not commute with the $\overline{\mathscr{P}}$ functor. Take $X$ obtained from the semiopen interval $[0,+\infty)$ by attaching one 1-sphere at each nonnegative integer. In this case, the natural map

$$
{ }^{p} H_{1}(X) \rightarrow \overline{\mathscr{P}}\left(\left(\text { pro } H_{1}, H_{1}\right) \varepsilon^{\prime} X\right)
$$

is not an isomorphism.

(3) We can also consider the following functor

$$
\text { Pro } \stackrel{S \varepsilon}{\longrightarrow} \operatorname{pro} S S, S S) \stackrel{f}{\longrightarrow}(\operatorname{pro} S A, S A) \stackrel{\mathscr{D}^{R}}{\longrightarrow} S\left(A_{\mathscr{P}_{c} \mathbb{Z}}\right) \stackrel{U}{\longrightarrow} S A,
$$

which induces another proper homology theory that also satisfies a Hurewicz theorem. In this case, the functor $H_{q}$ "commutes" with $\overline{\mathscr{P}}$.

(4) Otherwise useful proper invariants are the strong (Steenrod) homotopy groups of a rayed space that can be defined by $\pi_{q}\left(F_{\mathrm{sh}} \mathscr{P}^{R} \varepsilon^{\prime} X\right)$ or by $\pi_{q} \lim ^{R} \varepsilon^{\prime} X$ see [H-P.1, H-P.2]. Other alternative definition can be seen in [Če]. A proper homology theory for these groups, that satisfy the Hurewicz theorem, can be defined by $H_{q}\left(F_{\mathrm{sh}} \mathscr{P}^{R} \varepsilon^{\prime} X\right)$. Other Hurewicz theorems for the strong homotopy groups are proved in [E-H-R.2].

\section{APPLICATIONS TO PROHOMOTOPY THEORY}

In this section, in order to prove new versions of standard theorems for the homotopy category $\mathrm{Ho}\left(\right.$ pro $\left.S S_{*}\right)$, we will use the pair of adjoint functors

$$
\operatorname{Ho}\left(S\left(S_{* \mathscr{D} C S^{0}}\right)\right) \underset{\mathscr{D}^{R}}{\stackrel{\mathscr{D}^{L}}{\rightleftarrows}} \mathrm{Ho}\left(\text { pro } S S_{*}\right) \text {. }
$$

Definition 1. An object $X$ of pro $S S_{*}$ is said to be $\mathscr{L}$-cofibrant if $X$ is isomorphic in $\mathrm{Ho}\left(\right.$ pro $\left.S S_{*}\right)$ to some $\mathscr{L} G$, where $G$ is a cofibrant object in $S\left(S_{*} \mathscr{D}_{c} S^{0}\right)$. If $G$ is cofibrant and $\operatorname{dim} G \leq k$, then $X$ is said to be $\mathscr{L}$-k-cofibrant.

There are many versions of the Whitehead theorem in prohomotopy theory. On one side, there are theorems that give algebraic conditions to ensure that a morphism of pro Ho(Top) is an isomorphism-see for instance [Rau] and [MS]. On the other side, there are theorems of the same type for a morphism of $\mathrm{Ho}$ (pro Top). The monograph of Edwards and Hastings [E-H] and the papers 
of Grossman [Gr.1, Gr.2, Gr.3] include some versions of the last type for maps between towers that satisfy additional conditions on (co) dimension or movability. Here we prove a slightly different version of the Whitehead theorem for $\mathscr{L}$-cofibrant objects. In general, an $\mathscr{L}$-cofibrant object is not necessarily isomorphic to a tower. The algebraic condition of our result is given in terms of Grossman homotopy groups or equivalent cohomology conditions:

Theorem 1. Let $X, Y$ be $\mathscr{L}$-cofibrant objects in pro $S S_{*}$ and let $u: X \rightarrow Y$ be a map in $\mathrm{Ho}\left(\operatorname{pro} S S_{*}\right)$. If $\mathscr{P}^{R} u$ is an isomorphism in $\mathrm{Ho}\left(S\left(S_{* \mathscr{D} c S^{0}}\right)\right)$, then $u$ is an isomorphism in $\mathrm{Ho}\left(\operatorname{pro} S S_{*}\right)$.

Proof. If suffices to prove that for any cofibrant object $G$ of $S\left(S_{\left.\left(\mathscr{P}_{c} S^{0}\right)\right)}\right.$ the induced map

$$
u_{*}: \operatorname{Ho}\left(\operatorname{pro} S S_{*}\right)(\mathscr{L} G, X) \rightarrow \mathrm{Ho}\left(\operatorname{pro} S S_{*}\right)(\mathscr{L} G, Y)
$$

is an isomorphism. Because $\mathscr{L}^{L}$ is left adjoint to $\mathscr{P}^{R}$, this condition is equivalent to showing that

$$
\left(\mathscr{P}^{R} u\right)_{*}: \operatorname{Ho}\left(\left(S_{*} \mathscr{P}_{c} S^{0}\right)\right)\left(G, \mathscr{P}^{R} X\right) \rightarrow \operatorname{Ho}\left(\left(S_{* \mathscr{P} c S^{0}}\right)\right)\left(G, \mathscr{P}^{R} Y\right)
$$

is an isomorphism. This follows because $\mathscr{P}^{R} u$ is an isomorphism by hypothesis.

Consider a simplicial $q$-sphere, for instance $S^{q}=\dot{\Delta}[q+1]$, and recall that $\mathscr{L}\left(S^{q} \odot \mathscr{P} c S^{0}\right) \cong c S^{q}$. Given an object $X$ in pro $S S_{*}$, the $q$ th Grossman homotopy group

$$
\begin{aligned}
{ }^{G} \pi_{q}^{\infty}(X) & \cong \operatorname{Ho}\left(\left(S_{* \mathscr{P} c S^{0}}\right)\right)\left(S^{q} \odot \mathscr{P}_{c} S^{0}, \mathscr{P}^{R} X\right) \\
& \cong \operatorname{Ho}\left(S S_{*}\right)\left(S^{q}, \overline{\mathscr{P}}^{R} X\right) \cong \pi_{q}\left(\overline{\mathscr{P}}^{R} X\right) .
\end{aligned}
$$

A pro-pointed simplicial set is said to be (Grossman) 0 -connected if ${ }^{G} \pi_{0}^{\infty}(X)$ is trivial.

Corollary 1. Let $X, Y$ be $\mathscr{L}$-cofibrant objects in pro $S S_{*}$ and assume that $X$ and $Y$ are 0-connected $\left({ }^{G} \pi_{0}^{\infty}=0\right)$. If $u: X \rightarrow Y$ is a morphism in $\mathrm{Ho}\left(\operatorname{pro} S S_{*}\right)$, then $u$ is an isomorphism if and only if ${ }^{G} \pi_{q}^{\infty} X \rightarrow{ }^{G} \pi_{q}^{\infty} Y$ is an isomorphism for all $q \geq 1$.

Proof. By Theorem 1, $u$ is an isomorphism if and only if $\mathscr{P}^{R} u$ is an isomorphism. It is easy to check that $\mathscr{P}^{R} u$ is an isomorphism in $\operatorname{Ho}\left(S\left(S_{* \mathscr{P} c S^{0}}\right)\right)$ if and only if $\overline{\mathscr{P}}^{R} u=U \mathscr{P}^{R} u$ is an isomorphism in $\mathrm{Ho}\left(S S_{*}\right)$. We note that the simplicial sets $\overline{\mathscr{P}}^{R} X$ and $\mathscr{\mathscr { P }}^{R} Y$ are 0 -connected. Therefore this is equivalent to saying that $\pi_{q} \overline{\mathscr{P}}^{R} u$ is an isomorphism for $q \geq 1$. Since $\pi_{q} \overline{\mathscr{P}}^{R} u={ }^{G} \pi_{q}^{\infty} u$, we get the algebraic condition of the corollary.

Remark. We can define the cohomology of a prosimplicial set $X$ with twisted coefficients in $A$ by $H^{q}(X ; A):=H^{q}\left(\overline{\mathscr{P}}^{R} X ; A\right)$, where $A$ is a $\pi$-module and $\pi={ }^{G} \pi_{q}^{\infty} X$. It is clear that we can give a cohomology version of the Whitehead theorem for $\mathscr{L}$-cofibrant objects.

Recall that the natural "inclusion" $\mathrm{Ho}(S S) \rightarrow \mathrm{Ho}($ pro $S S)$ is left adjoint to the homotopy limit $\lim ^{R}: \mathrm{Ho}($ pro $S S) \rightarrow \mathrm{Ho}(S S)$, and for the case of towers $\lim ^{R}$ factors as $\lim ^{R}=F_{\mathrm{sh}} \mathscr{P}^{R}$. We also have similar functors and properties for the pointed case. 
Given an object $X$ in pro $S S_{*}$, the $q$ th strong homotopy group of $X$ is defined by $S_{\pi_{q}}(X)=\pi_{q}\left(\lim ^{R} X\right)$. If $X$ is a tower we have that $\pi_{q}\left(\lim ^{R} X\right)=$ $\pi_{q}\left(F_{\mathrm{sh}} \mathscr{P}^{R} X\right)$. The Čech homotopy groups of $X$ are defined by $\check{\pi}_{q}(X)=$ $\lim \pi_{q} X$, where $\pi_{q} X$ denotes the progroup pro $\pi_{q} X$. If $X$ is a tower then $\check{\pi}_{q}(X)=\lim \pi_{q} X \cong F_{\mathrm{sh}} \mathscr{P} \pi_{q} X \cong F_{\mathrm{sh}} \pi_{q} \mathscr{P}^{R} X$. Then the homotopy groups $\pi_{q} \mathscr{P}^{R} X$ determine the Čech homotopy groups $\check{\pi}_{q}(X)$ (the homotopy group $\pi_{q} \mathscr{P}^{R} X$ is provided in a natural way with a shift operator $\mathrm{sh}$ ).

For any object $X$ in pro $S S_{*}$, we have the natural map $\lim ^{R} X \rightarrow X$ in pro $S S_{*}$. Consider the following notion.

Definition 2. An object $X$ in pro $S S_{*}$ is said to be $\overline{\mathscr{P}}_{\text {-movable }}$ if the induced map $\mathscr{\mathscr { P }}^{R} \lim ^{R} X \rightarrow \overline{\mathscr{P}}^{R} X$ is a weak equivalence in $S S_{*}$.

Proposition 1. Let $X$ be an object of tow $S S_{*}$ and assume that $X$ is $\overline{\mathscr{P}}$. movable. Then

(i) The Cech homotopy groups $\check{\pi}_{q}(X)$ are isomorphic to the strong homotopy

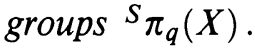

(ii) The strong homotopy groups determine the Grossman homotopy groups by the formula ${ }^{G} \pi_{q}^{\infty}(X) \cong \overline{\mathscr{P}}\left({ }^{S} \pi_{q}(X)\right)$.

Proof. To prove (i), consider the following isomorphisms:

$$
\begin{aligned}
\check{\pi}_{q}(X) & =\lim \pi_{q} X \cong F_{\mathrm{sh}} \mathscr{P} \pi_{q} X \cong F_{\mathrm{sh}} \pi_{q} \mathscr{P}^{R} X \\
& \cong F_{\mathrm{sh}} \pi_{q} \mathscr{P}^{R} \lim ^{R} X \cong F_{\mathrm{sh}} \mathscr{P}\left(\pi_{q} \lim ^{R} X\right) \cong \pi_{q} \lim ^{R} X \cong{ }_{\pi_{q}(X) .}
\end{aligned}
$$

Part (ii) follows from the isomorphisms

$$
{ }^{G} \pi_{q}^{\infty}(X)=\pi_{q}\left(\overline{\mathscr{P}}^{R} X\right) \cong \pi_{q}\left(\overline{\mathscr{P}}^{R} \lim ^{R} X\right) \cong \overline{\mathscr{P}}_{q} \lim ^{R} X \cong \overline{\mathscr{P}}\left({ }^{S} \pi_{q}(X)\right) .
$$

Now we obtain the following Whitehead theorem for $\overline{\mathscr{P}}_{\text {-movable prosimpli- }}$ cial sets.

Corollary 2. Let $X, Y$ be objects in tow $S S_{*}$ and assume that $X, Y$ are $\mathscr{L}$.

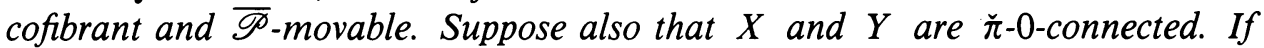
$u: X \rightarrow Y$ is a morphism in $\mathrm{Ho}\left(\operatorname{pro} S S_{*}\right)$, then the following conditions are equivalent:

(i) $u$ is an isomorphism in $\mathrm{Ho}\left(\operatorname{pro} S S_{*}\right)$.

(ii) $\check{\pi}_{q}(X) \rightarrow \check{\pi}_{q}(Y)$ is an isomorphism for all $q \geq 1$.

(iii) ${ }^{s} \pi_{Q}(X) \rightarrow{ }^{s_{q}}(Y)$ is an isomorphism for all $q \geq 1$.

Proof. Since $X, Y$ are $\overline{\mathscr{P}}$-movable, we infer by the proposition above that

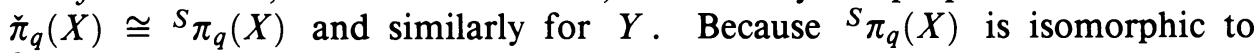
$s_{\pi_{q}}(Y)$, by the proposition above it follows that

$$
{ }^{G} \pi_{q}^{\infty}(X) \cong \overline{\mathscr{P}}\left({ }^{S} \pi_{q} X\right) \cong \overline{\mathscr{P}}\left({ }^{S} \pi_{q} Y\right) \cong{ }^{G} \pi_{q}^{\infty}(Y), \quad q \geq 1 .
$$

Applying Corollary 1 we have that condition (iii) implies that $u$ is an isomorphism.

Remarks. (1) Let $X$ be a topological space and $\alpha:[0,+\infty) \rightarrow X$ be a "proper" cofibration. In Remark (1) after Theorem 8.3 we have considered the pointed prosimplicial set $S \varepsilon^{\prime}(X, \alpha)$. We say that $X$ is $\overline{\mathscr{P}}$-movable at infinity if

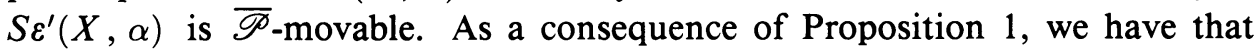


for a space $X$ which is $\overline{\mathscr{P}}$-movable at infinity, the strong (Steenoord) homotopy group

$$
s_{\pi_{q}(X, \alpha) \cong \pi_{0}\left(\operatorname{Pro}_{\infty}\right)\left(\left(S^{q} \times[0,+\infty), * \times[0,+\infty)\right),(X, \alpha)\right)}
$$

is isomorphic to the proper Čech group

$$
\check{\pi}_{q}(X, \alpha)=\lim \pi_{q} \varepsilon(X, \alpha) .
$$

Therefore we also have the following proper Whitehead theorem: Let $(X, \alpha)$ and $(Y, \beta)$ be two "well rayed" simplicial complexes (objects in PC) and let $f:(X, \alpha) \rightarrow(Y, \beta)$ be the germ of a proper map. Assume that (i) $X, Y$ have finite dimension, (ii) $X, Y$ are $\overline{\mathscr{P}}$-movable at infinity, and (iii) $X, Y$ have one Freudenthal end. Then $f$ is a proper homotopy equivalence at infinity if and only if $\check{\pi}_{q}(f): \check{\pi}_{q}(X, \alpha) \rightarrow \check{\pi}_{q}(Y, \beta)$ is an isomorphism for $q \geq 1$.

A Whitehead theorem involving only strong (Steenrod) proper homotopy groups was proved in [E-H-R.1].

(2) If we define the $H \overline{\mathscr{P}}$-homology groups of a prosimplicial set $X$ by $H^{\overline{\mathscr{P}}_{q}}(X)=H_{q}\left(\overline{\mathscr{P}}^{R} X\right)$, we also have a homology theory that satisfies the Hurewicz theorem for the Grossman homotopy groups. We will analyse this case in the following section for the prosimplicial set $V X$ associated with a compact metrisable space $X$.

(3) Many of the notions and theorems of this section can also be obtained for the nonpointed case pro $S S$, and the corresponding global (augmented) categories (pro $S S, S S),\left(\operatorname{pro} S S_{*}, S S_{*}\right)$.

\section{APPLiCATIONS to STRONG SHAPE THEORY}

First we recall the definitions of the Čech nerve $C X$ of a space $X$ and the Vietoris nerve $V X$ that was introduced by Porter [P.1].

Given a space $X$, consider the directed set $\operatorname{cov} X$. An element of $\operatorname{cov} X$ is an open covering $\mathscr{U}$ of $X$. If $\mathscr{U}, \mathscr{V} \in \operatorname{cov} X$, it is said that $\mathscr{V}$ refines $\mathscr{U}(\mathscr{V} \geq \mathscr{U})$ if for any $V \in \mathscr{V}$ there is some $U \in \mathscr{U}$ such that $V \subset U$. Given a space $X$ and an open covering $\mathscr{U},(C X)_{\mathscr{U}}$, denotes a simplicial set such that a typical $n$-simplex is given by $\left(U_{0}, \ldots, U_{n}\right)$ where $U_{0}, \ldots, U_{n} \in \mathscr{U}$ and $U_{0} \cap \cdots \cap U_{n} \neq \varnothing$. The correspondence $X \rightarrow\left\{(C X)_{U} \mid U \in \operatorname{cov} X\right\}$ defines a functor $C:$ Top $\rightarrow \operatorname{pro} \operatorname{Ho}(S S)$.

If $\mathscr{U}$ is an open covering of the space $X$, the Vietoris nerve of $\mathscr{U},(V X)_{\mathscr{U}}$, is the simplicial set in which an $n$-simplex is an ordered $(n+1)$-tuple $\left(x_{0}, \ldots\right.$, $\left.x_{n+1}\right)$ of points contained in an open set $U \in \mathscr{U}$. One important difference with the Čech nerve is that if $\mathscr{V}$ refines $\mathscr{U}$ there is a canonical map $(V X)_{\mathscr{V}} \rightarrow$ $(V X)_{\mathscr{U}}$ in $S S$, whereas in the case of the Čech nerve the corresponding map $(C X)_{\mathscr{V}} \rightarrow(C X)_{\mathscr{U}}$ has to be considered only in $\mathrm{Ho}(S S)$.

Using the Vietoris functor $V:$ Top $\rightarrow$ pro $S S$, one can define the category $\mathrm{St} \mathrm{Sh}$ (Top) of strong shape of topological spaces by taking as objects the topological spaces, and for two spaces $X, Y$ the hom-set $\operatorname{St} \operatorname{Sh}(X, Y)$ is defined by

$$
\operatorname{St} \operatorname{Sh}(X, Y)=\operatorname{Ho}(\operatorname{pro} S S)(V X, V Y),
$$

where pro $S S$ is provided with the closed model structures given by Edwards and Hastings [E-H]. We shall also use the Dowker theorem [E-H, page 125], 
which asserts that for an open covering $\mathscr{U}$ of a topological space the Vietoris nerve $(V X)_{\mathscr{U}}$ is isomorphic to the Čech nerve $(C X)_{\mathscr{U}}$ in the category $\operatorname{Ho}(S S)$.

It is not difficult to check that if $X$ is a compact metrisable space, then there is a cofinal sequence $\ldots, \mathscr{U}_{2}, \mathscr{U}_{1}, \mathscr{U}_{0}$ of open coverings in cov $X$. Therefore $C X=\left\{(C X)_{\mathscr{U}} \mid \mathscr{U} \in \operatorname{cov} X\right\}$ is isomorphic to $C^{\prime} X=\left\{(C X)_{\mathscr{U}_{i}} \mid i \in \mathbb{N}\right\}$ in pro $\mathrm{Ho}(S S)$ and $V X=\left\{(V X)_{\mathscr{U}} \mid \mathscr{U} \in \operatorname{cov} X\right\}$ is isomorphic to $V^{\prime} X=$ $\left\{(V X)_{\mathscr{U}_{i}} \mid i \in \mathbb{N}\right\}$ is pro $S S$ and in $\operatorname{Ho}(\operatorname{pro} S S)$.

Recall that if $X$ is a compact metrisable space we can assume (up to homomorphism) that $X$ is a subspace of $s=\prod_{n=1}^{+\infty}\left(\frac{-1}{n}, \frac{1}{n}\right)$, the pseudo-interior of the Hilbert cube $Q=\prod_{n=1}^{+\infty}\left[\frac{-1}{n}, \frac{1}{n}\right]$. Consider the open neighbourhoods of $X$ in $Q$

$$
N X=\{U \mid X \subset U, U \text { is an open subset of } Q\}
$$

as an object of pro Top. Since $X$ is a compact space, there is a cofinal sequence of neighbourhoods $N^{\prime} X=\left\{U_{i} \mid X \subset U_{i} i \in \mathbb{N}\right\}$ such that $N X$ is isomorphic to $N^{\prime} X$ in pro Top. Applying the singular functor we get $S N X=\{S U \mid U \in N X\}$, which is isomorphic to $V X$ in $\mathrm{Ho}(\operatorname{pro} S S)$. It is also interesting to remark that the natural inclusion

$$
N^{\prime c} X=\left\{U_{i}-X \mid X \subset U_{i}, \quad i \in \mathbb{N}\right\} \subset\left\{U_{i} \mid X \subset U_{i}, \quad i \in \mathbb{N}\right\}=N^{\prime} X
$$

is an isomorphism in $\mathrm{Ho}$ (pro Top) and therefore $S N^{\prime} X$ and $S N^{\prime c} X$ are isomorphic in $\mathrm{Ho}$ (pro $S S$ ).

Notice that by considering the functor $\mathrm{Ho}($ tow $S S) \rightarrow$ tow $\mathrm{Ho}(S S)$ and the Dowker theorem we have that, for a compact metrisable space $X, C^{\prime} X$ and $V^{\prime} X$ are isomorphic in tow $\mathrm{Ho}(S S)$. If we choose representative maps of the bounding maps of $C^{\prime} X$, we obtain an object $C^{\prime \prime} X$ in the category tow $S S$, and by Theorem 5.2.9 of [E-H] we also have that $C^{\prime \prime} X$ and $V^{\prime} X$ are isomorphic in Ho(tow $S S$ ). Therefore, for a compact metrisable space the objects $V X$, $S N X, V^{\prime} X, S N X, S N^{\prime c} X, C^{\prime \prime} X$ are isomorphic in $\mathrm{Ho}(\operatorname{pro} S S)$.

Recall that in the Example (2) of $\S 2$, we introduced the simplicial $M$-sets $s s(X)$ and $s s^{\mathcal{C}}(X)$ for a compact subset $X$ of the pseudo-interior of the Hilbert cube. The following result gives a geometric interpretation of the simplicial $M$ set $\mathscr{P}^{R} V X$.

Proposition 1. Let $X$ be a compact subset of the pseudo-interior of the Hilbert cube. Then $\mathscr{P}^{R} V X, s s(X)$, and $s s^{c}(X)$ are isomorphic in $\operatorname{Ho}\left(S\left(S_{M}\right)\right)$, where $M=\operatorname{Pro}(\mathbb{N}, \mathbb{N})$.

Proof. Since $V X$ is isomorphic to $S N X$, then

$$
\mathscr{P}^{R} V X \cong \mathscr{P}^{R} S N X \cong \mathscr{P}^{R} S N^{\prime} X \cong \mathscr{P}^{R} S N^{\prime c} X \text {. }
$$

The objects $S N^{\prime} X=\left\{S U_{i} \mid i \in \mathbb{N}\right\}$ and $S N^{\prime c} X=\left\{S\left(U_{i}-X\right) \mid i \in \mathbb{N}\right\}$ are such that for each $i \in \mathbb{N}, S U_{i}$ and $S\left(U_{i}-X\right)$ are fibrant in $S S$. By Lemma 6.3, we infer that $\mathscr{P}{ }^{R} S N^{\prime} X$ is isomorphic to $\mathscr{P} S N^{\prime} X$ and $\mathscr{P}{ }^{R} S N^{\prime C} X$ is isomorphic to $\mathscr{P} S N^{\prime c} X$ in the category $\operatorname{Ho}\left(S\left(S_{M}\right)\right)$. Now it is easy to check that $\mathscr{P} S N^{\prime} X$ is isomorphic to $s s(X)$ and $\mathscr{P} S N^{\prime c} X$ is isomorphic to $s s^{c}(X)$ in the category $S\left(S_{M}\right)$.

Remark. From the definition of $s s^{c}(X)$ and $S_{p}(Q-X)$, it is clear that $s s^{c}(X)=$ $S_{p}(Q-X)$. 
To define invariants for the strong shape category, consider the following functors:

$$
\mathrm{St} \mathrm{Sh}(C M) \stackrel{V}{\longrightarrow} \mathrm{Ho}(\text { pro } S S) \stackrel{\mathscr{P}^{R}}{\longrightarrow} \mathrm{Ho}\left(S\left(S_{M}\right)\right) \stackrel{U}{\longrightarrow} \mathrm{Ho}(S S),
$$

where $\operatorname{St} \operatorname{Sh}(C M)$ is the strong shape category for compact metrisable spaces and $M$ is the monoid $\mathscr{P}_{C *}$. Recall that we also use the notation $U \mathscr{P}^{R}=\overline{\mathscr{P}}^{R}$. We also note that a base point $*$ of a compact metrisable space determines a base point of $\overline{\mathscr{P}}^{R} V X$.

Definition 1. The $\pi \overline{\mathscr{P}}$-homotopy groups of a pointed compact metrisable space are defined by

$$
\pi_{q}^{\overline{\mathscr{P}}}(X)=\pi_{q}\left(\overline{\mathscr{P}}^{R} V X\right)
$$

and the $H \overline{\mathscr{P}}$-homology groups of $X$ (nonpointed) by

$$
H_{q}^{\overline{\mathscr{P}}}(X)=H_{q}\left(\overline{\mathscr{P}}^{R} V X\right) .
$$

Remarks. (1) The $\pi \overline{\mathscr{P}}$-homotopy groups $\pi_{q}^{\overline{\mathscr{P}}}(X)$ are isomorphic to the "inward" groups $Q_{\pi}^{I}(X)$ of Quigley [Quig, P.6].

(2) Notice that $\pi$ and $\mathscr{\mathscr { P }}$ commute; that is, $\pi_{q} \overline{\mathscr{P}}^{R} V X \cong \overline{\mathscr{P}}_{q} V X$, where $\pi_{q} V X$ denotes the homotopy progroup pro $\pi_{q} V X$.

(3) In general $H$ and $\overline{\mathscr{P}}$ do not commute; that is, $H_{q} \overline{\mathscr{P}}^{R} V X \not \overline{\mathscr{P}} H_{q} V X$, where $H_{q} V X$ denotes the pro-abelian group pro $H_{q} V X$.

(4) To define homology theories, we can consider functors into a category of simplicial objects in an abelian category. For instance, the free abelian functor $f:$ Set $\rightarrow$ Ab induces natural functors $f: S S \rightarrow S A, f: \operatorname{pro} S S \rightarrow \operatorname{pro} S A$, where $S A$ is the category of simplicial abelian groups. We also have the free functor $f: S\left(S_{\mathscr{P}_{C *}}\right) \rightarrow S\left(S_{\mathscr{P} C \mathbb{Z}}\right)$, where $S\left(A_{\mathscr{P} c \mathbb{Z}}\right)$ denotes the category of simplicial objects in $A_{\mathscr{C} C \mathbb{Z}}(\mathrm{Ab}=A)$. Therefore we have the following simplicial objects to define homology of a prosimplicial set $X$ :
(a) $f U \mathscr{P}^{R} X$ in $S A$,
(b) $U f \mathscr{P}^{R} X$ in $S A$,
(c) $U \mathscr{P}^{R} f X$ in $S A$,
(d) $f \mathscr{P}^{R} X$ in $S A \mathscr{P} c \mathbb{Z}$
(e) $\mathscr{P} R f X$ in $S A_{\mathscr{P} c \mathbb{Z}}$
(f) $f X$ in $\operatorname{pro} S A$.

For the cases (c) and (e) we have that $H$ and $\overline{\mathscr{P}}$ commute. The homology in cases $(\mathrm{d})$ and $(\mathrm{e})$ has a natural structure as a $\mathscr{P} c \mathbb{Z}$-module. Recall that $\mathscr{P} c \mathbb{Z}$ is the ring of locally finite matrices modulo the ideal of infinite matrices, see [F-W].

An immediate consequence of the definition is that the $H \overline{\mathscr{P}}$-homology satisfies the Hurewicz theorem for the inward groups of Quigley.

Theorem 1. Let $X$ be a compact metrisable space and assume that $X$ is $Q_{\pi^{I}-0 \text { - }}$

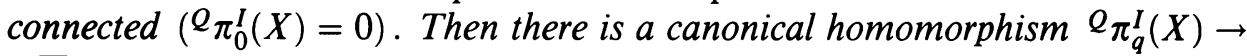
$H_{q}^{\overline{\mathscr{P}}}(X)$ such that

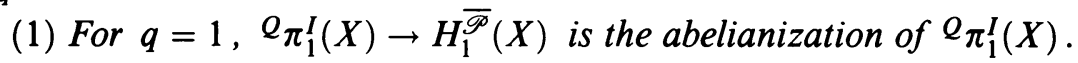




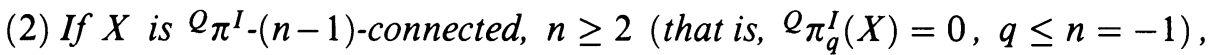
then $Q_{\pi_{n}^{I}}^{I}(X) \rightarrow H_{n}^{\overline{\mathscr{P}}}(X)$ is an isomorphism and $Q_{\pi_{n+1}^{I}}^{I}(X) \rightarrow H_{n+1}^{\overline{\mathscr{P}}}(X)$ is an epimorphism.

Proof. It suffices to apply the standard Hurewicz theorem to the simplicial set $\overline{\mathscr{P}}^{R} V X$.

Remark. There are other homologies that satisfy Hurewicz theorems for the inward groups of Quigley. For instance, consider the $H \overline{\mathscr{P}} f$-homology groups, $H_{q}^{\overline{\mathscr{P}} f}(X)=H_{q}\left(\overline{\mathscr{P}}^{R} f X\right)$.

It is also interesting to analyse the family of invariants obtained when one considers the commutative diagram

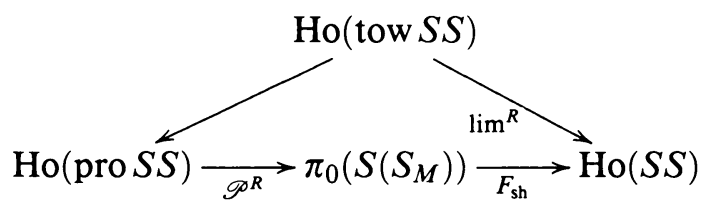

For a compact metrisable space the prosimplicial set $V X$ is isomorphism to a tower of simplicial sets; then holim $V X=\lim ^{R} V X \cong F_{\mathrm{sh}} \mathscr{P}^{R} V X$. That is, $\lim ^{R} V X$ is a sub-simplicial set of $\overline{\mathscr{P}}^{R} V X$. The inclusion $\lim ^{R} V X \subset \overline{\mathscr{P}}^{R} V X$ induces many relations between the homotopy invariants of $\lim ^{R} V X$ and the invariants of $\overline{\mathscr{P}}^{R} V X$.

Definition 2. The $\pi F \mathscr{P}$-homotopy groups of a pointed metrisable space are defined by

$$
\pi_{q}^{F \mathscr{P}}(X)=\pi_{q}\left(F_{\mathrm{sh}} \mathscr{P}^{R} V X\right)
$$

and the $H F \mathscr{P}$-homology groups of $X$ (nonpointed) by

$$
H_{q}^{F \mathscr{P}}(X)=H_{q}\left(F_{\mathrm{sh}} \mathscr{P}^{R} V X\right) .
$$

Remarks. (1) The $\pi F \mathscr{P}$-homotopy groups $\pi_{q}^{F \mathscr{P}}(X)$ are isomorphic to the approaching groups $Q_{\pi_{q}^{A}}^{A}(X)$ defined by Quigley [Quig, P.6].

(2) The functors $\pi_{q}$ and $F_{\text {sh }}$ do not commute. These are spaces $X$ such that $\pi_{q} F_{\mathrm{sh}} \mathscr{P}^{R} V S \not F_{\mathrm{sh}} \pi_{q} \mathscr{P}^{R} V X$. Notice that $F_{\mathrm{sh}} \pi_{q} \mathscr{P}^{R} V X \cong F_{\mathrm{sh}} \mathscr{P} \pi_{q} V X \cong$ $\lim \pi_{Q} V X$ is isomorphic to the Čech homotopy group $\check{\pi}_{q}(X)$. Therefore the $F \pi \mathscr{P}$-homotopy groups of $X,{ }^{F} \pi_{q}^{\mathscr{P}}(X)=F_{\text {sh }} \pi_{q} \mathscr{P} V X$ are up to isomorphism the Čech homotopy groups.

(3) We can consider the following simplicial objects, in different abelian categories, associated with a prosimplicial set $X$.

(a) $f F_{\mathrm{sh}} \mathscr{P}^{R}=f \lim ^{R} X$ in $S A$,

(b) $F_{\mathrm{sh}} f \mathscr{P}^{R} X$ in $S A$,

(c) $F_{\mathrm{sh}} \mathscr{P}^{R} f X=\lim ^{R} f X$ in $S A$.

The $H F \mathscr{P} f$-homology groups $($ or $H \lim f) H_{q}^{F \mathscr{P} f}(X)=H_{q}\left(F_{\mathrm{sh}} \mathscr{P}^{R} f X\right)$ are the strong (or Steenrod) homology groups ${ }^{S} H_{q}(X)$; see [E-H, page 208], [Co] and [P.4]. We can also consider $F \mathscr{P} H$-homology groups ${ }^{F \mathscr{P}} H_{q}(X)=F_{\mathrm{sh}} \mathscr{P} H_{q} X$ $\cong \lim H_{q} X \cong \lim$ pro $H_{q} X \cong \breve{H}_{q}(X)$ which are isomorphic to the Čech homology groups. 
There are theorems of Hurewicz type for the approaching groups $Q_{\pi_{q}^{A}(X)}$ of Quigley and the strong (Steenrod) homology groups ${ }^{s} H_{q}(X)$; see the paper of Kodama and Koyama [K-K]. We can also prove that the $H F \mathscr{P}$-homology groups satisfy a Hurewicz theorem for the approaching groups of Quigley.

Theorem 2. Let $X$ be a compact metrisable space and assume that $X$ is $Q_{\pi^{A}}$ 0 -connected (that is, $\left.Q_{\pi_{0}^{A}}(X)=0\right)$. Then there is a canonical homomorphism $Q_{\pi_{q}^{A}(X) \rightarrow H_{q}^{F \mathscr{P}}(X) \text { such that }}$

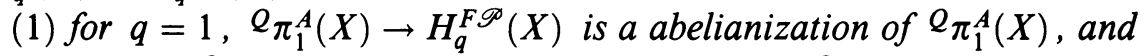

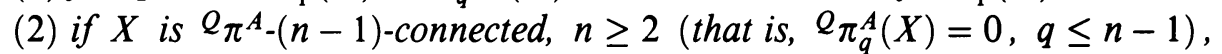
then $Q_{\pi_{n}^{A}}^{A}(X) \rightarrow H_{n}^{F \mathscr{P}}(X)$ is an isomorphism and $Q_{\pi_{n+1}^{A}}^{A}(X) \rightarrow \mathscr{H}_{n+1}^{F \mathscr{P}}(X)$ is an epimorphism.

Proof. This is a particular case of the standard Hurewicz theorem.

If $X$ is a compact metrisable space, then $V X$ is isomorphic to $C^{\prime \prime} X$ in $\mathrm{Ho}($ pro $S S)$. If the covering dimension of $X$ is finite, then $C^{\prime \prime} X$ is isomorphic to a tower of finite simplicial sets of dimension less than or equal to the covering dimension of $X$. It is not hard to check that a tower of finite simplicial sets of dimension $\leq n$ (for some $n$ ) is an $\mathscr{L}$-cofibrant object in the sense of Definition 9.1. Therefore if $X$ is a compact metrisable space and $X$ has finite covering dimension we have that $V X$ is an $\mathscr{L}$-cofibrant object. As a consequence of the Whitehead theorem proved in $\S 9$, we obtain the following version of the Whitehead theorem for the strong shape category.

Theorem 3. Let $X, Y$ be compact metrisable spaces with finite covering dimension. Assume also that $X$ and $Y$ are $Q_{\pi^{I}}$-0-connected $\left(Q_{0}^{I}=0\right)$. A strong shape morphism $f: X \rightarrow Y$ (that is, a map $f: V X \rightarrow V Y$ in Ho(pro $S S)$ ) is

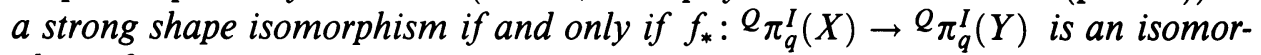
phism for $q \geq 1$.

Remarks. (1) For a compact metrisable space $X$, let $\pi=Q_{\pi_{1}^{I}(X)}$ be the fundamental inward group and let $A$ be a $\pi$-module. Define the cohomology of $X$ with twisted coefficient in $A$ by $H^{q}(X ; A)=H^{q}\left(\overline{\mathscr{P}}^{R} V X ; A\right)$. Then in Theorem 3 we can give the following equivalent condition:

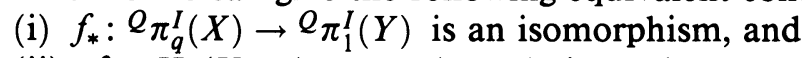

(ii) $f^{*}: H^{q}(Y ; A) \rightarrow H^{q}(X ; A)$ is an isomorphism for $q \geq 0$ and any twisted coefficients $A$.

(2) The functors $F_{\mathrm{sh}} \mathscr{P}^{R}$ and $\overline{\mathscr{P}}^{R}$ can be used to transform many notions and results of standard homotopy theory into strong shape notions and results. We have just included some canonical examples about Hurewicz and Whitehead theorems.

Definition 3. A compact metrisable space $X$ is said to be $\overline{\mathscr{P}}$-movable if $V X$ is $\overline{\mathscr{P}}_{\text {-movable (see Definition } 9.2 \text { ). }}$.

An immediate consequence of Corollary 9.2 is the following Whitehead theorem

Theorem 4. Let $X$ and $Y$ be compact metrisable spaces and assume that $X$ and $Y$ have finite covering dimension and that $X$ and $Y$ are $\mathscr{P}$-movable. Suppose also that $X, Y$ are $\check{\pi}$-0-connected $\left(\check{\pi}_{0}=0\right)$. If $f: X \rightarrow Y$ is a strong shape morphism the following conditions are equivalent: 
(i) $f$ is a strong shape isomorphism,

(ii) $\check{\pi}_{q}(X) \rightarrow \check{\pi}_{q}(Y)$ is an isomorphism for $q \geq 1$,

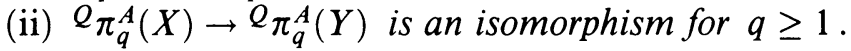

\section{ACKNOWLEDGMENTS}

I thank the referee for useful remarks and suggestions. I also acknowledge the financial help given by the University of Zaragoza and the University of La Rioja, and from the research projects "Programa Acciones Integradas Hispano-Germanas, 1991-127A" and "DGICYT, PB91-0861".

\section{REFERENCES}

[A-M] M. Artin and B. Mazur, Étale homotopy, Lecture Notes in Math., vol. 100, Springer, 1969.

[Ba.1] H. J. Baues, Algebraic homotopy, Cambridge Univ. Press, 1988.

[Ba.2] _ Foundations of proper homotopy theory, preprint (1992).

[Bas] D. Bassendoski, Whitehead and Hurewicz theorems in proper homotopy theory, Fakultät für Mathematik, Universität Bielefeld, 1977.

[B-K] A. K. Bousfield and D. M. Kan, Homotopy limits, completions and localizations, Lecture Notes in Math., vol. 304, Springer-Verlag, 1972.

[Br.1] E. M. Brown, On the proper homotopy type of simplicial complexes, Lecture Notes in Math., vol. 375, Springer, 1975.

[Če] Z. Čerin, On various relative proper homotopy groups, Tsukuba J. Math. 4 (1980), 177-202.

[Co] J.-M. Cordier, Homologie de Steenrod-Sitnikov et limite homotopique algébrique, Manuscripta Math. 59 (1987), 35-52.

[C-P] J.-M. Cordier and T. Porter, Shape theory, categorical methods of approximation, Ellis Horwood Ser. Math. Appl., Horwood, 1989.

[E-H] D. Edwards and H. Hastings, Čech and Steenrod homotopy theories with applications to geoemtric topology, Lecture Notes in Math., vol. 542, Springer, 1976.

[E-H-R.1] J. I. Extremiana, L. J. Hernández, and M. T. Rivas, Proper $C W$-complexes: A category for the study of proper homotopy, Collectanea Math. 39 (1988), 149-179.

[E-H-R.2] _ , An isomorphism theorem of the Hurewicz type in the proper homotopy category, Fund. Math. 132 (1989), 195-214.

[F-W.1] F. T. Farrell and J. B. Wagoner, Infinite matrices in algebraic $K$-theory and topology, Comment. Math. Helv. 47 (1972), 474-502.

[F-W.2] _ Algebraic torsion for infinite simple homotopy types, Comment. Math. Helv. 47 (1972), 502-513.

[F-T-W] F. T. Farrell, L. R. Taylor, and J. B. Wagoner, The Whitehead theorem in the proper category, Compositio Math. 27 (1973), 1-23.

[G-Z] P. Gabriel and M. Zisman, Calculus of fractions and homotopy theory, Springer, 1966.

[Gro] A. Grothendieck, Technique de descente et théorèmes d'existence en géométrie algébrique I-IV, Séminaire Bourbaki, Exposeés 190, 195, 212, 221, 1959-60, 1960-61.

[Gr.1] J. W. Grossman, A homotopy theory of pro-spaces, Trans. Amer. Math. Soc. 201 (1975), 161-176.

[Gr.2] _ _ Homotopy classes of maps between pro-spaces, Michigan Math. J. 21 (1974), 355-362.

[Gr.3] _ Homotopy groups of Pro-spaces, Illinois J. Math. 20 (1976), 622-625.

[He.1] L. J. Hernández, Embedding theorems for categories of prosets and progroups, preprint, 1993.

[H-P.1] L. J. Hernández and T. Porter, Proper pointed maps from $\mathbb{R}^{n+1}$ to a $\sigma$-compact space, Math. Proc. Cambridge Philos. Soc. 103 (1988), 457-462.

[H-P.2] , Global analogues of the Brown-Grossman proper homotopy groups, Math. Proc. Cambridge Philos. Soc. 104 (1988), 483-496. 
[K-K] Y. Kodama and A. Koyama, Hurewicz isomorphism theorem for Steenrod homology, Proc. Amer. Math. Soc. 74 (1979), 363-367.

[M] S. Mac Lane, Categories for the working mathematician, Springer-Verlag, 1971.

[M-M] S. Mac Lane and I. Moerdijk, Sheaves in geometry and logic, Springer-Verlag, 1991.

[M-S] S. Mardešić and J. Segal, Shape theory, North-Holland, 1982.

[Me1] J. D. P. Meldrum, Near-rings and their links with groups, Pitman, 1985.

[Mey] C. V. Meyer, Approximation filtrante de diagrammes finis par Pro-C, Ann. Sci. Math. Québec 4 (1979), 35-57.

[Pa] B. Pareigis, Categories and functors, Academic Press, 1970.

[P.1] T. Porter, Čech homotopy. I, J. London Math. Soc. (2) 6 (1973), 429-436.

[P.2] - Stabilitiy results for topological spaces, Math. Z. 140 (1974), 1-21.

[P.3] _ Abstract homotopy theory in procategories, Cahiers Topologie Géom. Différentielle Catégoriques, vol. 17, Univ. Picardie, 1976, pp. 113-124.

[P.4] - Coherent prohomotopical algebra, Cahiers Topologie Géom. Différentielle Catégoriques, vol. 18, Univ. Picardie, 1977, pp. 139-179.

[P.5] _ Coherent pro-homotopy theory, Cahiers Topologie Géom. Différentielle Catégoriques, vol. 19, Univ. Picardie, 1978, pp. 3-45.

[P.6] _ C C Cech and Steenrod homotopy and the Quigley exact couple in strong shape and proper homotopy theory, J. Pure Appl. Algebra 24 (1983), 303-312.

[Pilz] G. Pilz, Near-rings, North-Holland and American Elsevier, 1977.

[Q.1] D. Quillen, Homotopical algebra, Lecture Notes in Math., vol. 43, Springer, 1967.

[Q.2] - Rational homotopy theory, Ann. of Math. (2) 90 (1969), 205-295.

[Quig] J. B. Quigley, An exact sequence from the nth to the (n-1)-st fundamental group, Fund. Math. 77 (1973), 195-210.

[Rau] M. Rausen, Hurewicz isomorphism and Whitehead theorems in pro-categories, Arch. Math. (Basel) 30 (1978), 153-164.

[Sie.1] L. C. Siebenmann, The obstruction of finding a boundary for an open manifold of dimension greater than five, Thesis, 1965.

[Sie.2] _ Infinite simple homotopy types, Indag. Math. 32 (1970), 479-495.

[Ste] N. E. Steenrod, Regular cycles on compact metric spaces, Ann. of Math. (2) 41 (1940), 833-851.

[Str] A. Strøm, The homotopy category is a homotopy category, Arch. Math. (Basel) 23 (1972), 435-441.

Departamento de Matemáticas, Universidad de Zaragoza, 50009 Zaragoza, España E-mail address: zl@cc.unizar.es 